\title{
Root Systems and Generalized Associahedra
}

Sergey Fomin and Nathan Reading 

IAS/Park City Mathematics Series

Volume 14, 2004

\section{Root Systems and Generalized Associahedra}

\section{Sergey Fomin and Nathan Reading}

\section{|}

These lecture notes provide an overview of root systems, generalized associahedra, and the combinatorics of clusters. Lectures 1.2 cover classical material: root systems, finite reflection groups, and the Cartan-Killing classification. Lectures 34 provide an introduction to cluster algebras from a combinatorial perspective. Lecture 5 is devoted to related topics in enumerative combinatorics.

There are essentially no proofs but an abundance of examples. We label unproven assertions as either "lemma" or "theorem" depending on whether they are easy or difficult to prove. We encourage the reader to try proving the lemmas, or at least get an idea of why they are true.

For additional information on root systems, reflection groups and Coxeter groups, the reader is referred to $[\mathbf{9}, \mathbf{2 5}, \mathbf{3 4}$. For basic definitions related to convex polytopes and lattice theory, see [58 and [31, respectively. Primary sources on generalized associahedra and cluster combinatorics are [13, 19, 21]. Introductory surveys on cluster algebras were given in $\mathbf{2 2}, \mathbf{5 6}, \mathbf{5 7}$.

Note added in press (February 2007): Since these lecture notes were written, there has been much progress in the general area of cluster algebras and Catalan combinatorics of Coxeter groups and root systems. We have not attempted to update the text to reflect these most recent advances. Instead, we refer the reader to the online Cluster Algebras Portal, maintained by the first author.

Department of Mathematics, University of Michigan, Ann Arbor, MI 48109-1109, USA.

E-mail address: fomin@umich.edu, nreading@umich.edu.

This work was partially supported by NSF grants DMS-0245385 (S.F.) and DMS-0202430 (N.R.).

(C)2007 Sergey Fomin and Nathan Reading 


\section{Acknowledgments}

We thank Christos Athanasiadis, Jim Stasheff and Andrei Zelevinsky for careful readings of earlier versions of these notes and for a number of editorial suggestions, which led to the improvement of the paper.

S.F.: I am grateful to the organizers of the 2004 Graduate Summer School at Park City (Ezra Miller, Vic Reiner, and Bernd Sturmfels) for the invitation to deliver these lectures, and for their support, understanding, and technical help.

Sections 3.3 3.4 and Lecture 4 present results of an ongoing joint project with Andrei Zelevinsky centered around cluster algebras.

N.R.: I would like to thank Vic Reiner for teaching the course which sparked my interest in Coxeter groups; Anders Björner and Francesco Brenti for making a preliminary version of their forthcoming book available to the students in Reiner's course; and John Stembridge, whose course and lecture notes have deepened my knowledge of Coxeter groups and root systems.

Some of the figures in these notes are inspired by figures produced by Satyan Devadoss, Vic Reiner and Rodica Simion. Several figures were borrowed from 13, 19, 20, 21, 23. 


\section{LECTURE 1 Reflections and Roots}

\subsection{The pentagon recurrence}

Consider a sequence $f_{1}, f_{2}, f_{3}, \ldots$ defined recursively by $f_{1}=x, f_{2}=y$, and

$$
f_{n+1}=\frac{f_{n}+1}{f_{n-1}} \text {. }
$$

Thus, the first five entries are

$$
x, y, \frac{y+1}{x}, \frac{x+y+1}{x y}, \frac{x+1}{y} .
$$

Unexpectedly, the sixth and seventh entries are $x$ and $y$, respectively, so the sequence is periodic with period five! We will call (11) the pentagon recurrence 1

This sequence has another important property. A priori, we can only expect its terms to be rational functions of $x$ and $y$. In fact, each $f_{i}$ is a Laurent polynomial (actually, with nonnegative integer coefficients). This is an instance of what is called the Laurent phenomenon.

It will be helpful to represent this recurrence as the evolution of a "moving window" consisting of two consecutive terms $f_{i}$ and $f_{i+1}$ :

$$
\left[\begin{array}{l}
f_{1} \\
f_{2}
\end{array}\right] \stackrel{\tau_{1}}{\longrightarrow}\left[\begin{array}{l}
f_{3} \\
f_{2}
\end{array}\right] \stackrel{\tau_{2}}{\longrightarrow}\left[\begin{array}{l}
f_{3} \\
f_{4}
\end{array}\right] \stackrel{\tau_{1}}{\longrightarrow}\left[\begin{array}{l}
f_{5} \\
f_{4}
\end{array}\right] \stackrel{\tau_{2}}{\longrightarrow}\left[\begin{array}{l}
f_{5} \\
f_{6}
\end{array}\right] \longrightarrow \cdots,
$$

where the maps $\tau_{1}$ and $\tau_{2}$ are defined by

$$
\tau_{1}:\left[\begin{array}{l}
f \\
g
\end{array}\right] \longmapsto\left[\begin{array}{c}
\frac{g+1}{f} \\
g
\end{array}\right] \quad \text { and } \quad \tau_{2}:\left[\begin{array}{l}
f \\
g
\end{array}\right] \longmapsto\left[\begin{array}{c}
f \\
\frac{f+1}{g}
\end{array}\right] .
$$

Both $\tau_{1}$ and $\tau_{2}$ are involutions: $\tau_{1}^{2}=\tau_{2}^{2}=1$, where 1 denotes the identity map. The 5 -periodicity of the recurrence (10) translates into the identity $\left(\tau_{2} \tau_{1}\right)^{5}=1$. That is, the group generated by $\tau_{1}$ and $\tau_{2}$ is a dihedral group with 10 elements.

Let us now consider a similar but simpler pair of maps. Throw away the +1 's that occur in the definitions of $\tau_{1}$ and $\tau_{2}$, and take logarithms. We then obtain a pair of linear maps

$$
s_{1}:\left[\begin{array}{l}
x \\
y
\end{array}\right] \longmapsto\left[\begin{array}{c}
y-x \\
y
\end{array}\right] \text { and } s_{2}:\left[\begin{array}{l}
x \\
y
\end{array}\right] \longmapsto\left[\begin{array}{c}
x \\
x-y
\end{array}\right] .
$$

A (linear) hyperplane in a vector space $V$ is a linear subspace of codimension 1. A (linear) reflection is a map that fixes all the points in some linear hyperplane, and has an eigenvalue of -1 . The maps $s_{1}$ and $s_{2}$ are linear reflections satisfying $\left(s_{2} s_{1}\right)^{3}=1$. Thus, the group $\left\langle s_{1}, s_{2}\right\rangle$ is a dihedral group with 6 elements.

We are led to wonder if the dihedral behavior of $\left\langle\tau_{1}, \tau_{2}\right\rangle$ is related to, or even explained by the dihedral behavior of $\left\langle s_{1}, s_{2}\right\rangle$. To test this unlikely-sounding hypothesis, let us try to find similar examples. What other pairs $\left(s, s^{\prime}\right)$ of linear

1 The discovery of this recurrence and its 5-periodicity are sometimes attributed to R. C. Lyness (1942); see, e.g., [15. It was probably already known to N. H. Abel. This recurrence is closely related to (and easily deduced from) the famous "pentagonal identity" for the dilogarithm function, first obtained by W. Spence (1809) and rediscovered by Abel (1830) and C. H. Hill (1830). See, e.g., 37 . 
reflections generate finite dihedral groups? To keep things simple, we set $s=s_{1}$ and confine the choice of $s^{\prime}$ to maps of the form

$$
s^{\prime}:\left[\begin{array}{l}
x \\
y
\end{array}\right] \longmapsto\left[\begin{array}{c}
x \\
L(x, y)
\end{array}\right],
$$

where $L$ is a linear function. Keeping in mind that $s_{1}$ and $s_{2}$ arose as logarithms, we require that $L$ have integer coefficients.

After some work, one determines that besides $x-y$, the functions $2 x-y$ and $3 x-y$ are the only good choices for $L$. More specifically, define

$$
s_{3}:\left[\begin{array}{l}
x \\
y
\end{array}\right] \longmapsto\left[\begin{array}{c}
x \\
2 x-y
\end{array}\right] \text { and } s_{4}:\left[\begin{array}{l}
x \\
y
\end{array}\right] \longmapsto\left[\begin{array}{c}
x \\
3 x-y
\end{array}\right] .
$$

Then $\left(s_{3} s_{1}\right)^{4}=1$ and $\left(s_{4} s_{1}\right)^{6}=1$. Thus, $\left\langle s_{1}, s_{3}\right\rangle$ and $\left\langle s_{1}, s_{4}\right\rangle$ are dihedral groups with 8 and 12 elements, respectively.

By analogy with (3), we next define

$$
\tau_{3}:\left[\begin{array}{l}
f \\
g
\end{array}\right] \longmapsto\left[\begin{array}{c}
f \\
\frac{f^{2}+1}{g}
\end{array}\right] \quad \text { and } \quad \tau_{4}:\left[\begin{array}{l}
f \\
g
\end{array}\right] \longmapsto\left[\begin{array}{c}
f \\
\frac{f^{3}+1}{g}
\end{array}\right] .
$$

Calculations show that $\left(\tau_{3} \tau_{1}\right)^{6}=1$, and the group $\left\langle\tau_{1}, \tau_{3}\right\rangle$ is dihedral with 12 elements. We can think of $\tau_{1}$ and $\tau_{3}$ as defining a "moving window" for the sequence

$$
x, y, \frac{y+1}{x}, \frac{x^{2}+(y+1)^{2}}{x^{2} y}, \frac{x^{2}+y+1}{x y}, \frac{x^{2}+1}{y}, x, y, \ldots
$$

Notice that the Laurent phenomenon holds: these rational functions are Laurent polynomials - again, with nonnegative integer coefficients.

Likewise, $\left(\tau_{4} \tau_{1}\right)^{8}=1$, the group $\left\langle\tau_{1}, \tau_{4}\right\rangle$ is dihedral with 16 elements, and $\tau_{1}$ and $\tau_{4}$ define an 8-periodic sequence of Laurent polynomials.

In the first two lectures, we will develop the basic theory of finite reflection groups that will include their complete classification. This theory will later help explain the periodicity and Laurentness of the sequences discussed above, and provide appropriate algebraic and combinatorial tools for the study of other similar recurrences.

\subsection{Reflection groups}

Our first goal will be to understand the finite groups generated by linear reflections in a vector space $V$. It turns out that for such a group, it is always possible to define a Euclidean structure on $V$ so that all of the reflections in the group are ordinary orthogonal reflections. The study of groups generated by orthogonal reflections is a classical subject, which goes back to the classification of Platonic solids by the ancient Greeks.

Let $V$ be a Euclidean space. In what follows, all reflecting hyperplanes pass through the origin, and all reflections are orthogonal. A finite reflection group is a finite group generated by some reflections in $V$. In other words, we choose a collection of hyperplanes such that the group of orthogonal transformations generated by the corresponding reflections is finite. Infinite reflection groups are also interesting, but in these lectures, "reflection group" will always mean a finite one.

The set of reflections in a reflection group $W$ is typically larger than a minimal set of reflections generating $W$. This is illustrated in Figure 1.1, where $W$ is the group of symmetries of a regular pentagon. This 10-element group is generated by 
two reflections $s$ and $t$ whose reflecting lines make an angle of $\pi / 5$. It consists of 5 reflections, 4 rotations, and the identity element. In Figure 1.1. each of the 5 lines is labeled by the corresponding reflection.

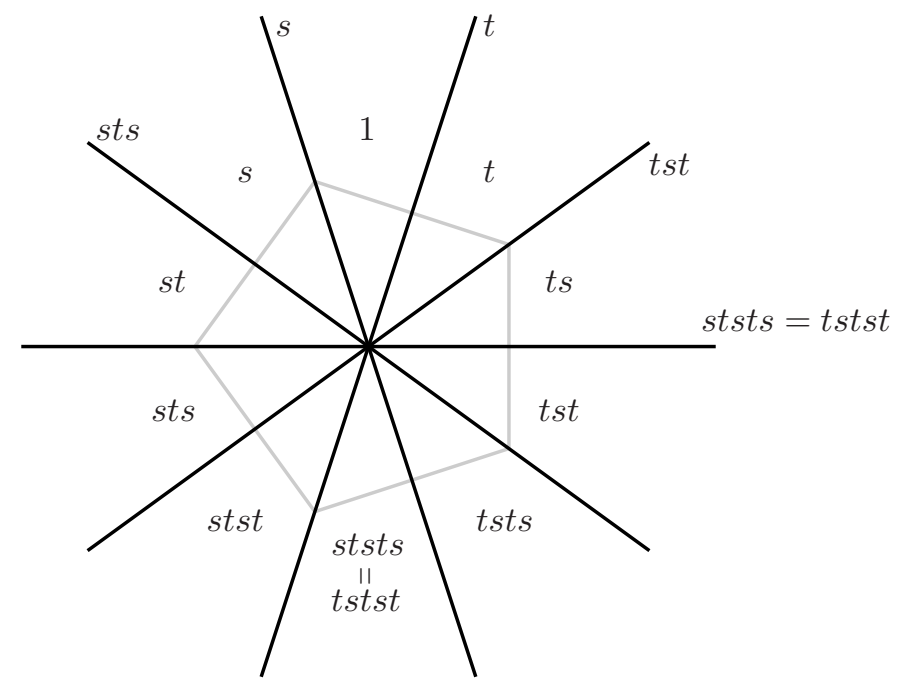

Figure 1.1. The reflection group $I_{2}(5)$.

Lemma 1.1. If $t$ is the reflection fixing a hyperplane $H$ and $w$ an orthogonal transformation, then $w t w^{-1}$ is the reflection fixing the hyperplane $w H$.

Lemma 1.2. Let $W$ be a finite group generated by a finite set $T$ of reflections. Then the set of all reflections in $W$ is $\left\{w t w^{-1}: w \in W, t \in T\right\}$.

The set $\mathcal{H}$ of all reflecting hyperplanes of a reflection group $W$ is called a Coxeter arrangement. In light of Lemmas 1.1 and 1.2. one can give an alternate definition of a Coxeter arrangement: A Coxeter arrangement is a collection $\mathcal{H}$ of hyperplanes which is closed under reflections in the hyperplanes. Like any hyperplane arrangement in $V$, a Coxeter arrangement cuts $V$ into connected components called regions. That is, the regions are the connected components of the complement to the union of all hyperplanes in $\mathcal{H}$.

The regions are in one-to-one correspondence with the elements of $W$, as follows. Once and for all, fix an arbitrary region $R_{1}$ to represent the identity element.

Lemma 1.3. The map $w \mapsto R_{w} \stackrel{\text { def }}{=} w\left(R_{1}\right)$ is a bijection between a reflection group $W$ and the set of regions of the corresponding Coxeter arrangement.

To illustrate, each of the 10 regions in Figure 1.1 is labeled by the corresponding element of the group.

The choice of a region representing the identity element leads to a distinguished choice of a minimal set of generating reflections. The facet hyperplanes of $R_{1}$ are the hyperplanes in $\mathcal{H}$ whose intersection with the closure of $R_{1}$ has dimension $n-1$.

Lemma 1.4. The reflections in the facet hyperplanes of $R_{1}$ generate $W$. This generating set is minimal by inclusion. 


\subsection{Symmetries of regular polytopes}

A regular polytope in a Euclidean space is a convex polytope whose symmetry group (i.e., the group of isometries of the space that leave the polytope invariant) acts transitively on complete flags of faces, i.e., on nested collections of the form

$$
\text { vertex } \subset \text { edge } \subset 2 \text {-dim. face } \subset \cdots
$$

Theorem 1.5. The symmetry group of any regular polytope is a reflection group.

The converse is false - see Remark 1.12

We illustrate Theorem 1.5 with several concrete examples.

Example 1.6. Consider a regular $m$-gon on a Euclidean plane, centered at the origin. The symmetry group of the $m$-gon is denoted by $I_{2}(m)$. This group contains (and is generated by) $m$ reflections, which correspond to the $m$ lines of reflective symmetry of the $m$-gon.

The group $I_{2}(m)$ is a dihedral group with $2 m$ elements. It is generated by two reflections $s$ and $t$ satisfying $(s t)^{m}=1$. To define $s$ and $t$, we use the construction of Lemma 1.4. Pick a side of the polygon, and consider two reflecting lines: one perpendicular to the side and another passing through one of its endpoints. The case $m=5$ is shown in Figure 1.1

Example 1.7. Take a regular tetrahedron in 3-space, with the vertices labeled 1, 2,3 , and 4 . Its symmetry group is obviously isomorphic to the symmetric group $\mathcal{S}_{4}$, which consists of the permutations of the set $\{1,2,3,4\}$. For each edge of the tetrahedron, choose a plane which is perpendicular to the edge and contains the other two vertices. Reflections in these six hyperplanes generate the symmetry group.

In general, the symmetry group of a regular simplex can be described as follows. Let $\left(e_{1}, \ldots, e_{n+1}\right)$ be the standard basis in $\mathbb{R}^{n+1}$. The standard $n$-dimensional simplex (or $n$-simplex) is the convex hull of the endpoints of the vectors $e_{1}, \ldots, e_{n+1}$. Thus the standard 1-simplex is a line segment in $\mathbb{R}^{2}$, the standard 2-simplex is an equilateral triangle in $\mathbb{R}^{3}$, and the standard 3-simplex is the regular tetrahedron described above, sitting in $\mathbb{R}^{4}$. The symmetry group $A_{n}$ of the standard $n$-simplex is canonically isomorphic to $\mathcal{S}_{n+1}$, the symmetric group of permutations of the set $[n+1] \stackrel{\text { def }}{=}\{1,2, \ldots, n+1\}$.

For each edge $\left[e_{i}, e_{j}\right]$ of the standard simplex, there is a hyperplane $x_{i}-x_{j}=0$ perpendicular to the edge and containing all the other vertices. Reflection through this hyperplane interchanges the endpoints of the edge and fixes the rest of the vertices. These $\left(\begin{array}{c}n+1 \\ 2\end{array}\right)$ reflections generate $A_{n}$.

To construct a minimal generating set of reflections, we again use Lemma 1.4 Let $R_{1}$ be the connected component of the complement to the $\left(\begin{array}{c}n+1 \\ 2\end{array}\right)$ reflecting hyperplanes defined by

$$
R_{1}=\left\{x_{1}<x_{2}<\cdots<x_{n+1}\right\} .
$$

The facet hyperplanes of $R_{1}$ are given by the equations

$$
x_{i}-x_{i+1}=0, \quad \text { for } i=1, \ldots, n .
$$

Then Lemma 1.4 reduces to the well-known fact that the symmetric group $\mathcal{S}_{n+1}$ is generated by the adjacent transpositions $s_{1}, \ldots, s_{n}$. (Here each $s_{i}$ exchanges $i$ and $i+1$, keeping everything else in its place.)

Figure 1.2 illustrates the special case $n=2$, the symmetry group of the standard 2 -simplex (shaded). The plane of the page represents the plane $x+y+z=1$ in $\mathbb{R}^{3}$. 


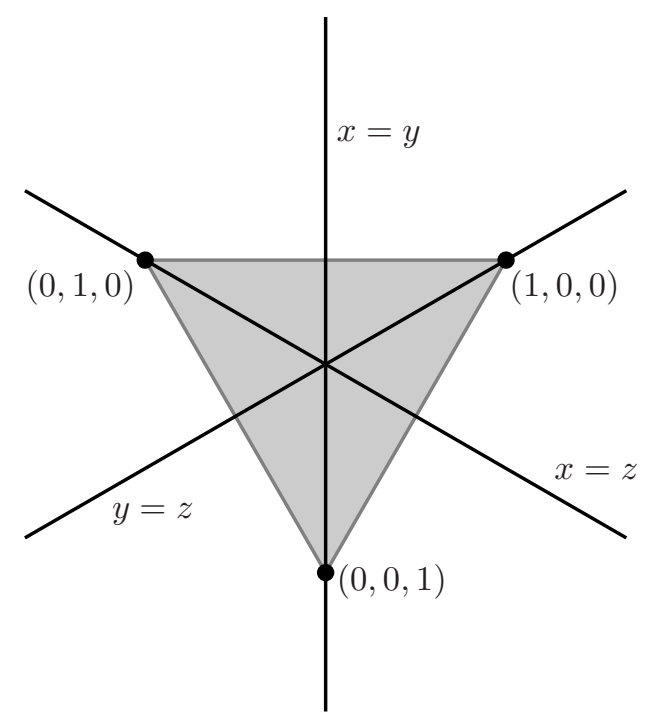

Figure 1.2. The reflection group $A_{2}$.

Example 1.8. The $n$-crosspolytope is the convex hull of (the endpoints of) the vectors $\pm e_{1}, \pm e_{2}, \ldots, \pm e_{n}$ in $\mathbb{R}^{n}$. For example, the 3 -crosspolytope is the regular octahedron. The symmetry group of this polytope is the hyperoctahedral group $B_{n}$. As in the previous examples, it is generated by the reflections it contains.

The special case $n=3$ (the symmetry group $B_{3}$ of a regular octahedron) is shown in Figure 1.3. The dotted lines show the intersections of reflecting hyperplanes with the front surface of the octahedron. Each edge of the octahedron is also contained in a reflecting plane.

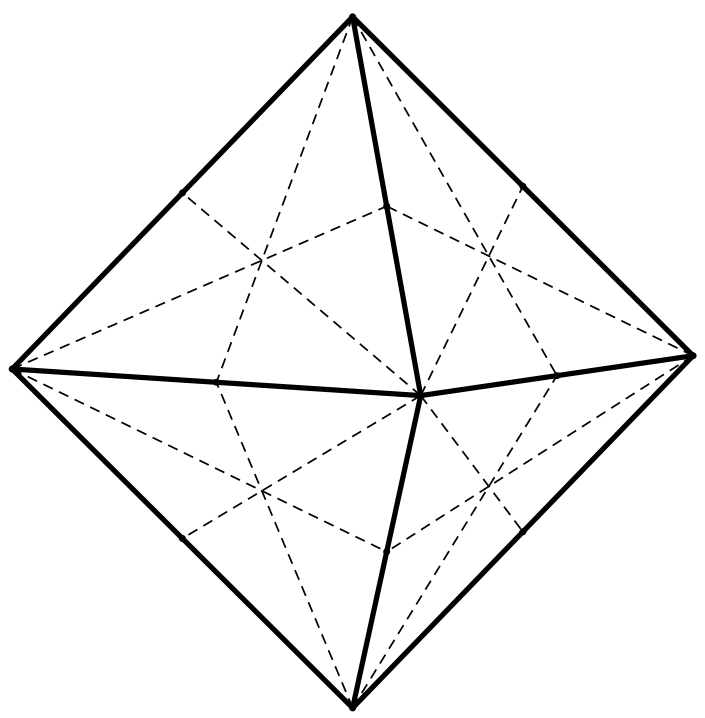

Figure 1.3. The reflection group $B_{3}$ 
There are two types of reflections in the symmetry group of the crosspolytope. One type of reflection transposes a vertex with its negative and fixes all other vertices. Also, for each pair $i \neq j$, there is a reflection which transposes $e_{i}$ and $e_{j}$, transposes $-e_{i}$ and $-e_{j}$, and fixes all other vertices.

To construct a minimal set of reflections generating $B_{n}$, take the minimal generating set for $A_{n-1}$ given in Example 1.7 and adjoin the reflection that interchanges $e_{1}$ and $-e_{1}$.

The group $B_{n}$ is also the symmetry group of the $n$-dimensional cube.

Example 1.9. The symmetry group of a regular dodecahedron (or a regular icosahedron) is the reflection group $H_{3}$. Figure 1.4 shows the dodecahedron and a minimal set of three reflections generating its symmetry group. The dotted lines show the intersections of the corresponding three hyperplanes with the front surface of the dodecahedron.

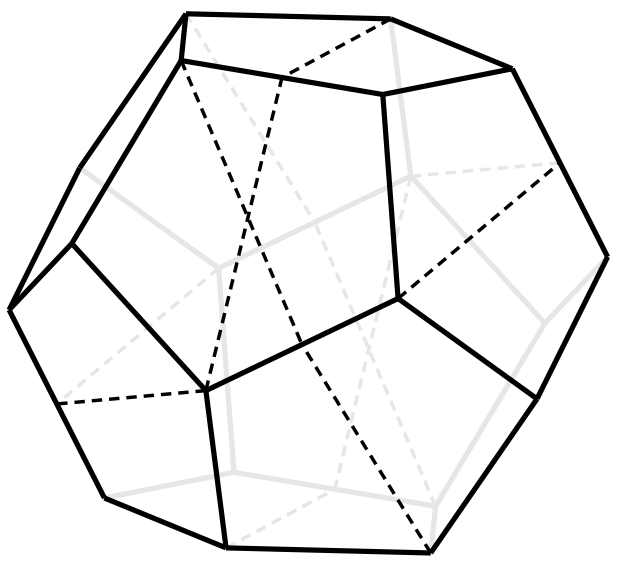

Figure 1.4. The reflection group $H_{3}$

Example 1.10. In 4-space, there are six types of regular polytopes. The obvious three are the 4-simplex, the 4-cube, and the 4-crosspolytope. There are two regular polytopes whose symmetry group is the reflection group called $H_{4}$. One of these, the 120 -cell, has 600 vertices and 120 dodecahedral faces; the other, the 600-cell, has 120 vertices and 600 tetrahedral faces. The remaining regular 4-dimensional polytope is the 24 -cell, with 24 vertices and 24 octahedral faces. Its symmetry group is a reflection group denoted by $F_{4}$.

Not every reflection group is the symmetry group of a regular polytope. A counterexample is constructed as follows.

Example 1.11. Let $n \geq 3$. Returning to the crosspolytope, ignore the reflections which transpose an opposite pair of vertices. The remaining reflections generate a reflection group called $D_{n}$, which is a proper subgroup of $B_{n}$. The reflections of $D_{3}$ are represented by the dotted lines in Figure 1.3. We note that the Coxeter arrangements of types $A_{3}$ and $D_{3}$ are related by an orthogonal transformation, so the reflection groups $A_{3}$ and $D_{3}$ are isomorphic to each other.

Remark 1.12. It can be shown that, for $n \geq 4$, the group $D_{n}$ is not a symmetry group of a regular polytope. See Section 2.3 for further details. 


\subsection{Root systems}

Root systems are configurations of vectors obtained by replacing each reflecting hyperplane of a reflection group by a pair of opposite normal vectors; the resulting configuration should be invariant under the action of the group. Here is a formal definition. A finite root system is a finite non-empty collection $\Phi$ of nonzero vectors in $V$ called roots with the following properties:

(i) Each one-dimensional subspace of $V$ either contains no roots, or contains two roots $\pm \alpha$.

(ii) For each $\alpha \in \Phi$, the reflection $\sigma_{\alpha}$ permutes $\Phi$.

The following lemma shows that the study of root systems is essentially equivalent to the study of reflection groups.

Lemma 1.13. For a finite root system $\Phi$, the group generated by the reflections $\left\{\sigma_{\alpha}: \alpha \in \Phi\right\}$ is finite. The corresponding reflecting hyperplanes form a Coxeter arrangement. Conversely, for any reflection group $W$, there is a root system $\Phi$ such that the orthogonal reflections $\left\{\sigma_{\alpha}\right\}_{\alpha \in \Phi}$ are precisely the reflections in $W$.

In Section 1.2, we fixed a region $R_{1}$ of the associated Coxeter arrangement $\mathcal{H}$. The simple roots in $\Phi$ are the roots normal to the facet hyperplanes of $R_{1}$ and pointing into the half-space containing $R_{1}$. The rank of $\Phi$ is the cardinality $n$ of the set of simple roots $\Pi$. Since $W$ acts transitively on the regions of $\mathcal{H}$, the rank of $\Phi$ does not depend on the choice of $\Pi$, and is equal to the dimension of the linear span of $\Phi$. It will be convenient to fix an indexing set $I$ so that $\Pi=\left\{\alpha_{i}: i \in I\right\}$. The standard choice is $I=[n]=\{1, \ldots, n\}$.

For any $\alpha \in \Phi$, the coefficients $c_{i}$ in the expansion $\alpha=\sum_{i \in I} c_{i} \alpha_{i}$ are called the simple root coordinates of $\alpha$. The set $\Phi_{+}$of positive roots consists of all roots whose simple root coordinates are all non-negative. The negative roots $\Phi_{-}$are those with non-positive simple root coordinates.

Lemma 1.14. $\Phi$ is the disjoint union of $\Phi_{+}$and $\Phi_{-}$.

In these lectures, we focus on the study of the important class of finite crystallographic root systems. These are the finite non-empty collections of vectors that, in addition to the axioms (i)-(ii) above, satisfy the "crystallographic condition"

(iii) For any $\alpha, \beta \in \Phi$, we have $\sigma_{\alpha}(\beta)=\beta-a_{\alpha \beta} \alpha$ with $a_{\alpha \beta} \in \mathbb{Z}$. (See Figure 1.5.) Equivalently, the simple root coordinates of any root are integers.

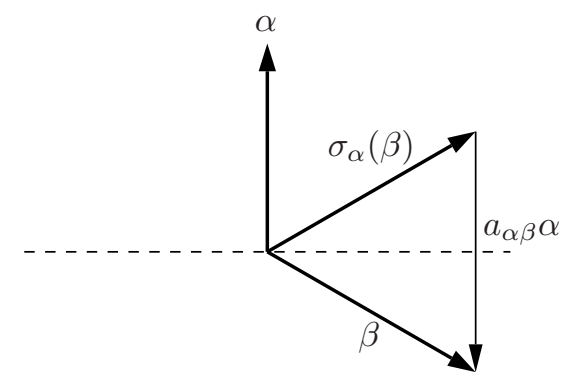

Figure 1.5. Reflecting $\beta$ in the hyperplane perpendicular to $\alpha$.

For the rest of these lectures, a "root system" will always be presumed finite and crystallographic. 
Example 1.15. A root system of rank 1 is called $A_{1}$; it consists of a pair of vectors $\pm \alpha$. There are four non-isomorphic (finite crystallographic) root systems of rank 2, called $A_{1} \times A_{1}, A_{2}, B_{2}$ and $G_{2}$; see Figure 1.6

$$
\begin{gathered}
A_{1} \times A_{1} \\
\sigma_{\alpha_{1}}=\left[\begin{array}{rr}
-1 & 0 \\
0 & 1
\end{array}\right] \\
\sigma_{\alpha_{2}}=\left[\begin{array}{rr}
1 & 0 \\
0 & -1
\end{array}\right]
\end{gathered}
$$

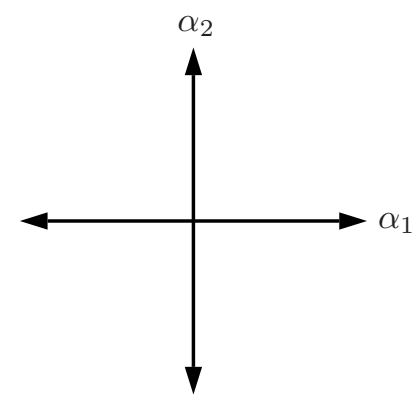

$$
\begin{gathered}
A_{2} \\
\sigma_{\alpha_{1}}=\left[\begin{array}{rr}
-1 & 1 \\
0 & 1
\end{array}\right] \\
\sigma_{\alpha_{2}}=\left[\begin{array}{rr}
1 & 0 \\
1 & -1
\end{array}\right]
\end{gathered}
$$

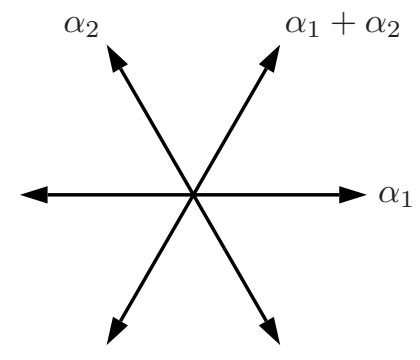

$$
\begin{gathered}
B_{2} \\
\sigma_{\alpha_{1}}=\left[\begin{array}{rr}
-1 & 2 \\
0 & 1
\end{array}\right] \\
\sigma_{\alpha_{2}}=\left[\begin{array}{rr}
1 & 0 \\
1 & -1
\end{array}\right]
\end{gathered}
$$
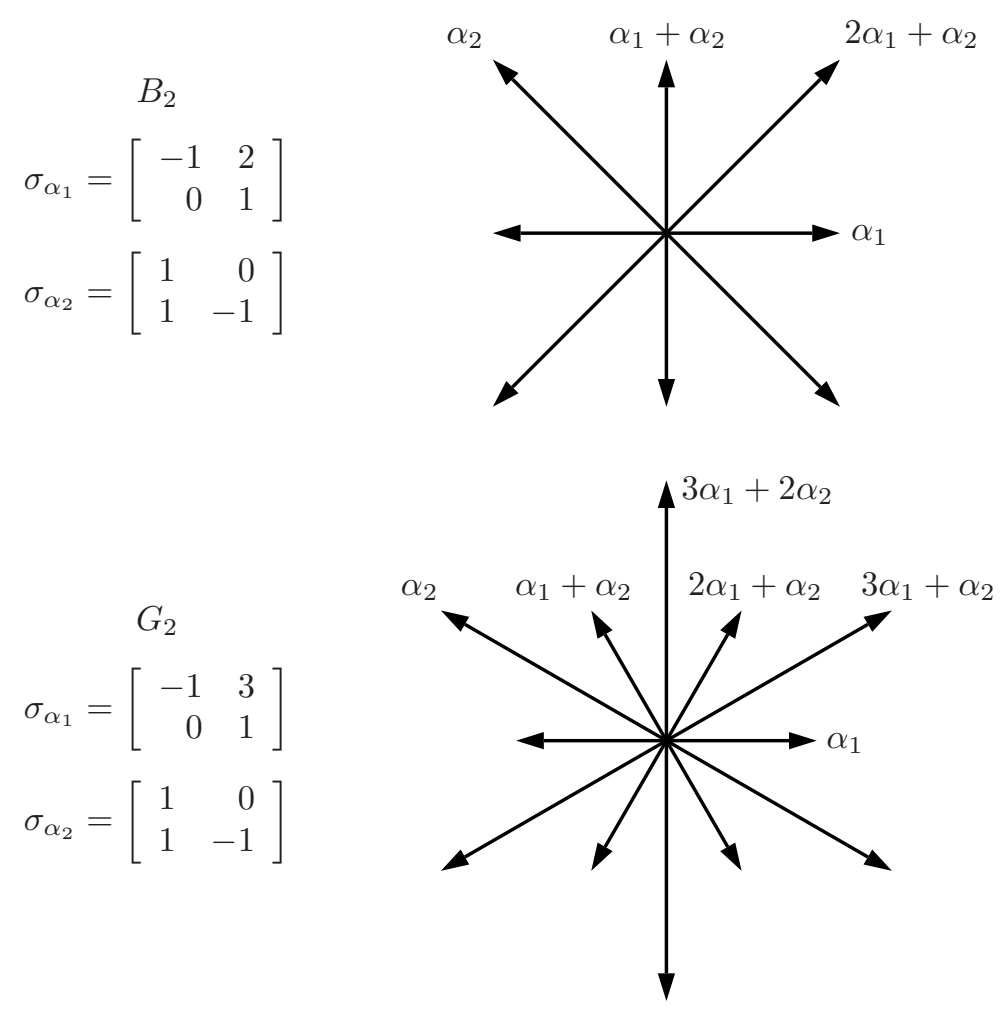

Figure 1.6. The finite crystallographic root systems of rank 2 
For the root systems $A_{2}, B_{2}$ and $G_{2}$, the reflections $\sigma_{\alpha_{1}}$ and $\sigma_{\alpha_{2}}$ have appeared earlier in Section 1.1. (The matrices of these reflections in the basis $\left(\alpha_{1}, \alpha_{2}\right)$ of simple roots are shown in Figure [1.6. ) In these three cases, the pair $\left(\sigma_{\alpha_{1}}, \sigma_{\alpha_{2}}\right)$ coincides with $\left(s_{2}, s_{1}\right),\left(s_{3}, s_{1}\right)$, and $\left(s_{4}, s_{1}\right)$, respectively, in the notation of Section 1.1 .

\subsection{Root systems of types A, B, C, and D}

Here we present four classical families of root systems, traditionally denoted by $A_{n}$, $B_{n}, C_{n}$ and $D_{n}$. The corresponding reflection groups have types $A_{n}, B_{n}, B_{n}$ and $D_{n}$ (cf. Examples 1.7 1.8, and 1.11). In each case, $n$ is the rank of a root system.

We realize each root system inside a Euclidean space with a fixed orthonormal basis $\left(e_{1}, e_{2}, \ldots\right)$, and describe particular choices of the sets of simple and positive roots. There is no "canonical" way to make these choices. Our realizations of root systems coincide with those in [9, 34, but our choices of simple/positive roots (which are motivated by notational convenience alone) are different.

\section{The root system $A_{n}$}

The root system $A_{n}$ can be realized as the set of vectors $e_{i}-e_{j}$ in $\mathbb{R}^{n+1}$ with $i \neq j$. Let $R_{1}$ be given by (5). Then the $n$ simple roots are $\alpha_{i} \stackrel{\text { def }}{=} e_{i+1}-e_{i}$, for $i=1, \ldots, n$, and the positive roots are $e_{i}-e_{j}$, for $1 \leq j<i \leq n+1$.

Figure 1.7 shows a planar projection of the root system $A_{3}$. The positive roots are labeled by their simple root coordinates. The solid lines are in the plane of the page. Thick dotted lines are above the plane, while thin dotted lines are below it.

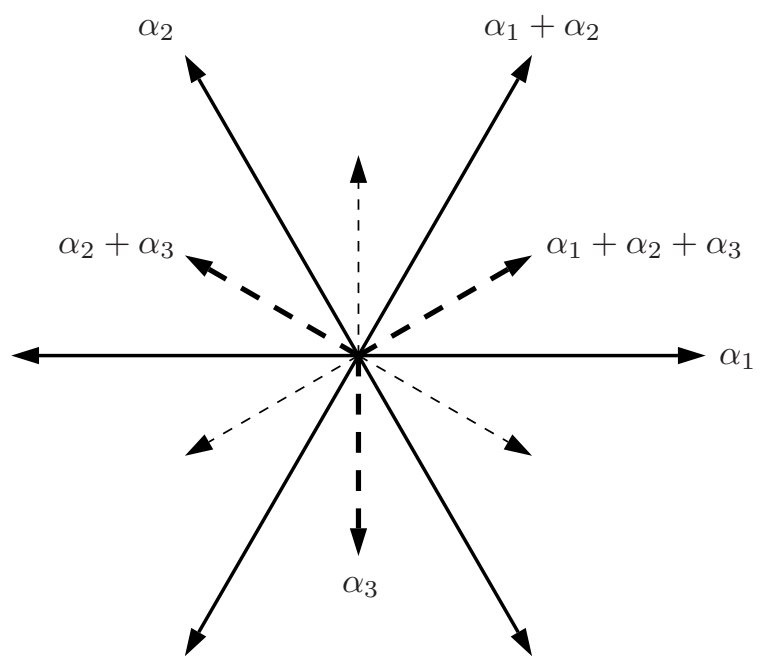

Figure 1.7. The root system $A_{3}$

The root systems $B_{n}$ and $C_{n}$

The root system $B_{n}$ can be realized as the set of vectors in $\mathbb{R}^{n}$ of the form $\pm e_{i}$ or $\pm e_{i} \pm e_{j}$ with $i \neq j$. Choose $R_{1}=\left\{0<x_{1}<x_{2}<\cdots<x_{n}\right\}$. Then the vectors $\alpha_{0}=e_{1}$ and $\alpha_{i}=e_{i+1}-e_{i}$ for $i \in[n-1]$ form a set of simple roots. The positive roots are $e_{i}$ for $i \in[n]$ and $e_{i} \pm e_{j}$ for $1 \leq j<i \leq n$. See Figure 1.8. 


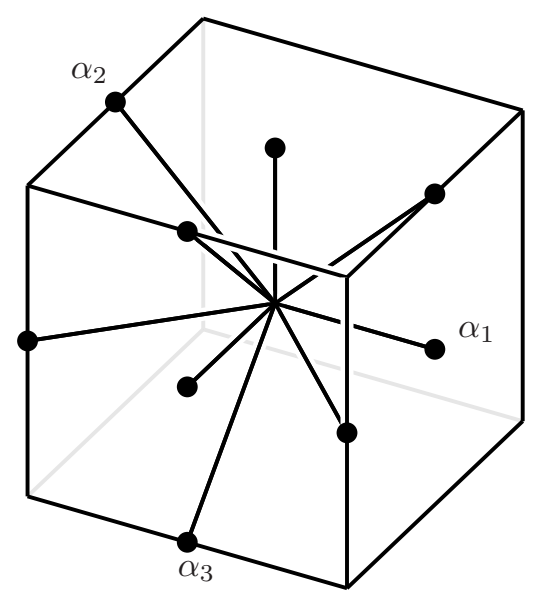

Figure 1.8. The root system $B_{3}$. The endpoints of the 9 positive roots are shown as black circles on the cube's front. The negative roots are not shown.

The root system $C_{n}$ can be realized as the set of vectors in $\mathbb{R}^{n}$ of the form $\pm 2 e_{i}$ or $\pm e_{i} \pm e_{j}$. The vectors $\alpha_{0}=2 e_{1}$ and $\alpha_{i}=e_{i+1}-e_{i}$ form a set of simple roots. The positive roots are $2 e_{i}$ and $e_{i} \pm e_{j}$. See Figure 1.9 ,

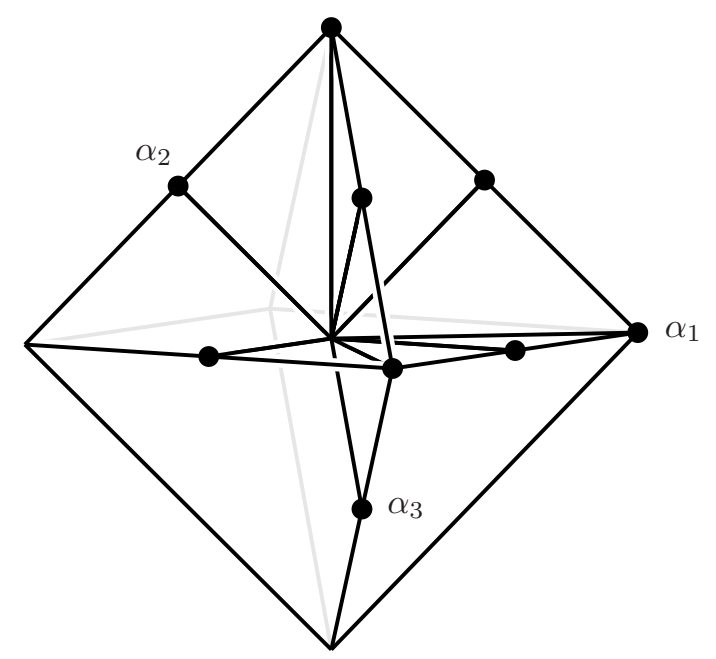

Figure 1.9. The root system $C_{3}$. The endpoints of the 9 positive roots are shown on the front of the octahedron. The negative roots are not shown.

The root system $C_{n}$ is a rescaling of $B_{n}$, so the corresponding reflection groups $W$ coincide. In contrast to the type $A_{n}$, the action of $W$ on the roots of $B_{n}$ or $C_{n}$ is not transitive: there are two orbits, corresponding to two different lengths of roots.

The root system $D_{n}$

The root system $D_{n}$ can be realized as the vectors $\pm e_{i} \pm e_{j}$ with $i \neq j$. One choice of simple roots is $\alpha_{0}=e_{2}+e_{1}$ and $\alpha_{i}=e_{i+1}-e_{i}$, giving the positive roots $e_{i} \pm e_{j}$ for $1 \leq j<i \leq n$. This comes from setting $R_{1}=\left\{-x_{2}<x_{1}<x_{2}<\cdots<x_{n}\right\}$. 


\section{LECTURE 2 Dynkin Diagrams and Coxeter Groups}

\subsection{Finite type classification}

The most fundamental result in the theory of (finite crystallographic) root systems is their complete classification, obtained by W. Killing and E. Cartan in late nineteenth - early twentieth century. (See the historical notes in [9].) To present this classification, we will need a few preliminaries.

First, we will need the notion of isomorphism. The ambient space $Q_{\mathbb{R}}=Q_{\mathbb{R}}(\Phi)$ of a root system $\Phi$ is the real span of $\Phi$. It inherits a Euclidean structure from $V$. Root systems $\Phi$ and $\Phi^{\prime}$ are isomorphic if there is an isometry map $Q_{\mathbb{R}}(\Phi) \rightarrow Q_{\mathbb{R}}\left(\Phi^{\prime}\right)$ of their ambient spaces that sends $\Phi$ to some dilation $c \Phi^{\prime}$ of $\Phi^{\prime}$.

The Cartan matrix of a root system $\Phi$ is the integer matrix $\left[a_{i j}\right]_{i, j \in I}$, where $a_{i j}$ is such that $\sigma_{\alpha_{i}}\left(\alpha_{j}\right)=\alpha_{j}-a_{i j} \alpha_{i}$, as in part (iii) of the definition of a root system. (This convention agrees with [21, 35] but is "transposed" to the one in [9, 34.)

Lemma 2.1. Root systems $\Phi$ and $\Phi^{\prime}$ are isomorphic if and only if they have the same Cartan matrix, up to simultaneous rearrangement of rows and columns.

Example 2.2. The Cartan matrices for the root systems of rank two are:

$$
\begin{array}{rlrl}
A_{1} \times A_{1}: & {\left[\begin{array}{ll}
2 & 0 \\
0 & 2
\end{array}\right]} & A_{2}: & {\left[\begin{array}{rr}
2 & -1 \\
-1 & 2
\end{array}\right]} \\
B_{2}: & {\left[\begin{array}{rr}
2 & -2 \\
-1 & 2
\end{array}\right]} & G_{2}:\left[\begin{array}{rr}
2 & -3 \\
-1 & 2
\end{array}\right]
\end{array}
$$

Example 2.3. The Cartan matrices for the root systems of type $A_{4}, B_{4}, C_{4}$, and $D_{4}$ are, respectively:

$$
\begin{aligned}
A_{4}: & {\left[\begin{array}{rrrr}
2 & -1 & 0 & 0 \\
-1 & 2 & -1 & 0 \\
0 & -1 & 2 & -1 \\
0 & 0 & -1 & 2
\end{array}\right] \quad B_{4}: \quad\left[\begin{array}{rrrr}
2 & -2 & 0 & 0 \\
-1 & 2 & -1 & 0 \\
0 & -1 & 2 & -1 \\
0 & 0 & -1 & 2
\end{array}\right] } \\
C_{4}: & {\left[\begin{array}{rrrr}
2 & -1 & 0 & 0 \\
-2 & 2 & -1 & 0 \\
0 & -1 & 2 & -1 \\
0 & 0 & -1 & 2
\end{array}\right] \quad D_{4}:\left[\begin{array}{rrrr}
2 & 0 & -1 & 0 \\
0 & 2 & -1 & 0 \\
-1 & -1 & 2 & -1 \\
0 & 0 & -1 & 2
\end{array}\right] }
\end{aligned}
$$


The Cartan matrices of (finite crystallographic) root systems are sometimes called Cartan matrices of finite type. This class of matrices is completely characterized by several elementary properties.

Theorem 2.4. An integer $n \times n$ matrix $\left[a_{i j}\right]$ is a Cartan matrix of a root system if and only if

(i) $a_{i i}=2$ for every $i$;

(ii) $a_{i j} \leq 0$ for any $i \neq j$, with $a_{i j}=0$ if and only if $a_{j i}=0$;

(iii) there exists a diagonal matrix $D$ with positive diagonal entries such that $D A D^{-1}$ is symmetric and positive definite.

Remark 2.5. Condition (iii) can be replaced by

(iii') there exists a diagonal matrix $D^{\prime}$ with positive integer diagonal entries such that $D^{\prime} A$ is symmetric and positive definite.

Example 2.6. For the root systems $A_{1} \times A_{1}$ and $A_{2}$, the $2 \times 2$ identity matrix serves as $D$. For $B_{2}$ and $G_{2}$, take $D=\left[\begin{array}{cc}1 & 0 \\ 0 & \sqrt{2}\end{array}\right]$ and $D=\left[\begin{array}{cc}1 & 0 \\ 0 & \sqrt{3}\end{array}\right]$, respectively.

The characterization in Theorem 2.4 can be used to completely classify the Cartan matrices of finite type, or the corresponding root systems. It turns out that each of those is built from blocks taken from a certain relatively short list. Let us be more precise.

A root system $\Phi$ is called reducible if $\Phi$ is a disjoint union of root systems $\Phi_{1}$ and $\Phi_{2}$ such that every $\beta_{1} \in \Phi_{1}$ is normal to every $\beta_{2} \in \Phi_{2}$. If such a decomposition does not exist, $\Phi$ is called irreducible. The parallel definition for Cartan matrices is that a Cartan matrix of finite type is indecomposable if its rows and columns cannot be simultaneously rearranged to bring the matrix into block-diagonal form with more than one block.

The Cartan matrices of finite type can be encoded by their Dynkin diagrams. The vertices of a Dynkin diagram are labeled by the elements of the indexing set $I$; thus they are in bijection with the simple roots. Each pair of vertices $i$ and $j$ is then connected as shown below (with the vertex $i$ on the left):

$$
\begin{array}{ll}
\bullet & \text { if } a_{i j}=a_{j i}=0 \\
\bullet & \text { if } a_{i j}=a_{j i}=-1 \\
\Longleftrightarrow & \text { if } a_{i j}=-1 \text { and } a_{j i}=-2 \\
\Longleftrightarrow & \text { if } a_{i j}=-1 \text { and } a_{j i}=-3
\end{array}
$$

(It follows from Theorem 2.4 that these are the only possible pairs of values for $a_{i j}$ and $a_{j i}$. Cf. Example 2.2.)

Lemma 2.7. A Cartan matrix of finite type (resp., a root system) is indecomposable (resp., irreducible) if and only if its Dynkin diagram is connected.

Theorem 2.8 (Cartan-Killing classification of irreducible root systems and Cartan matrices of finite type). The complete list of Dynkin diagrams of irreducible root systems is presented in Figure 2.1. 


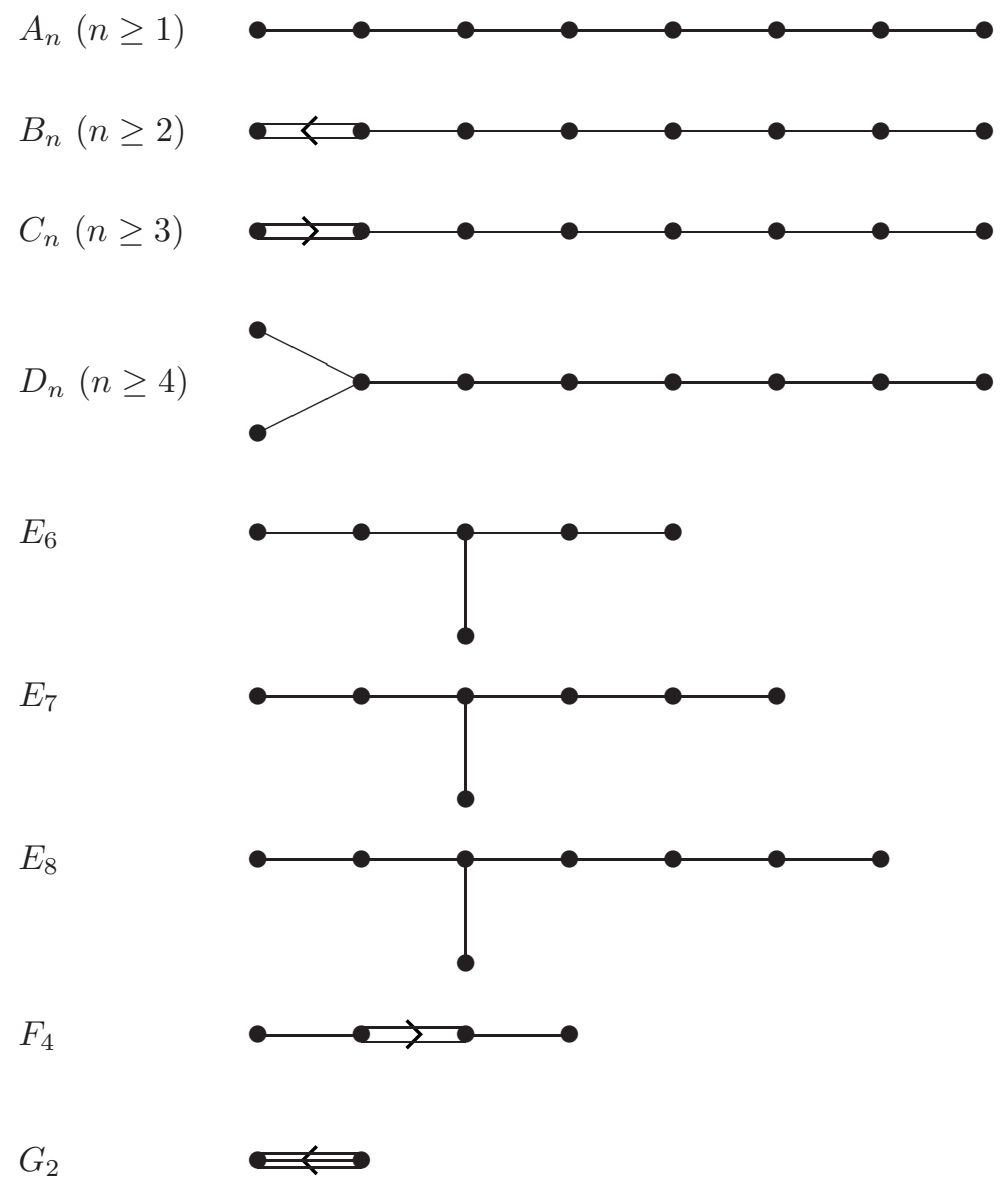

Figure 2.1. Dynkin diagrams of finite irreducible root systems.

Root systems are just one example among a large number of mathematical objects of "finite type" which are classified by (some class of) Dynkin diagrams. The appearance of the ubiquitous Dynkin diagrams in a variety of seemingly unrelated classification problems has fascinated several generations of mathematicians, and helped establish nontrivial connections between different areas of mathematics. See Section 2.3 and references therein.

\subsection{Coxeter groups}

Let $\Phi$ be a (finite crystallographic) root system and $\alpha \neq \beta$ a pair of roots in $\Phi$. The angle between the corresponding reflecting hyperplanes is a rational multiple of $\pi$ with denominator $2,3,4$ or 6 . Thus the rotation $\sigma_{\alpha} \sigma_{\beta}$ has order 2,3 , 4 , or 6 as an element of the associated reflection group $W$. The insight that the order of a product of reflections is directly related to the angle between the corresponding hyperplanes leads to the definition of a Coxeter group. 
Definition 2.9. A Coxeter system $(W, S)$ is a pair consisting of a group $W$ together with a finite subset $S \subset W$ satisfying the following conditions:

(i) each $s \in S$ is an involution: $s^{2}=1$;

(ii) some pairs $\{s, t\} \subset S$ satisfy relations of the form $(s t)^{m_{s t}}=1$ with $m_{s t} \geq 2$;

(iii) the relations in (i)-(ii) form a presentation of the group $W$.

In other words, $S$ generates $W$, and any identity in $W$ is a formal consequence of (i)-(ii) and the axioms of a group.

A group $W$ is called a Coxeter group if it has a presentation of the above form.

The following theorem demonstrates that the notion of a Coxeter group indeed captures the geometric essence of reflection groups.

Theorem 2.10. Any finite Coxeter group is isomorphic to a reflection group.

Conversely, a reflection group associated with a (finite crystallographic) root system $\Phi$ is a Coxeter group, in the following sense. Let $\Pi$ be the set of simple roots

in $\Phi$. For each simple root $\alpha_{i} \in \Pi$, the associated simple reflection is $s_{i} \stackrel{\text { def }}{=} \sigma_{\alpha_{i}}$.

Theorem 2.11. Let $W$ be the group generated by the reflections $\left\{\sigma_{\beta}: \beta \in \Phi\right\}$. Let

$$
S=\left\{s_{i}\right\}_{i \in I}=\left\{\sigma_{\alpha}: \alpha \in \Pi\right\}
$$

be the set of simple reflections. Then $(W, S)$ is a Coxeter system.

Furthermore, $W$ is a crystallographic Coxeter group, where the adjective "crystallographic" refers to restricting the integers $m_{s t}$ to the set $\{2,3,4,6\}$.

\subsection{Other "finite type" classifications}

The classification of root systems is similar or identical to several other classifications of objects of "finite type," briefly reviewed below.

\section{Non-crystallographic root systems}

Lifting the crystallographic restriction does not allow very many additional root systems. The only non-crystallographic irreducible finite root systems are those of types $H_{3}, H_{4}$ and $I_{2}(m)$ for $m=5$ or $m \geq 7$. See [34.

\section{Coxeter groups and reflection groups}

By Theorems 2.10 and 2.11 the classification of finite Coxeter groups is parallel to the classification of reflection groups and is essentially the same as the classification of root systems. The difference is that the root systems $B_{n}$ and $C_{n}$ correspond to the same Coxeter group $B_{n}$. A Coxeter group is encoded by its Coxeter diagram, a graph whose vertex set is $S$, with an edge $s-t$ whenever $m_{s t}>2$. If $m_{s t}>3$, the edge is labeled by $m_{s t}$. Figure 2.2 shows the Coxeter diagrams of the finite irreducible Coxeter systems, including the non-crystallographic Coxeter groups $H_{3}$, $H_{4}$ and $I_{2}(m)$. The group $G_{2}$ appears as $I_{2}(6)$. See [34 for more details. 


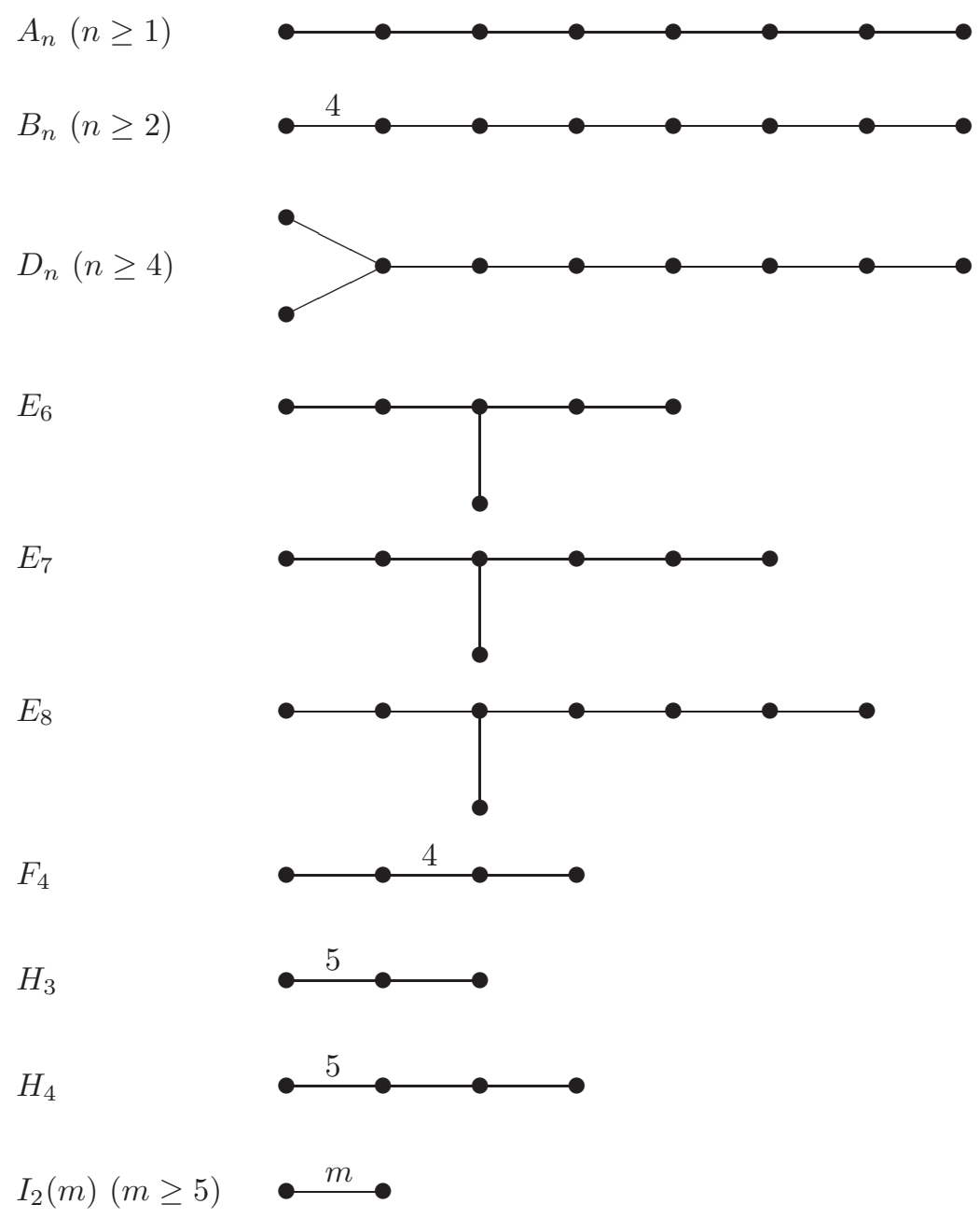

Figure 2.2. Coxeter diagrams of finite irreducible Coxeter systems

\section{Regular polytopes}

By Theorem 1.5 the symmetry group of a regular polytope is a reflection group. In fact, it is a Coxeter group whose Coxeter diagram is linear: the underlying graph is a path with no branching points. This narrows down the possibilities, leading to the conclusion that there are no other regular polytopes besides the ones described in Section 1.2. In particular, there are no "exceptional" regular polytopes beyond dimension 4: only simplices, cubes, and crosspolytopes. See [14.

\section{Lie algebras}

The original motivation for the Cartan-Killing classification of root systems came from Lie theory. Complex finite-dimensional simple Lie algebras correspond naturally, and one-to-one, to finite irreducible crystallographic root systems. There exist innumerable expositions of this classical subject; see, e.g., [25. 


\section{Quivers of finite type}

A quiver is a directed graph; its representation assigns a complex vector space to each vertex, and a linear map to each directed edge. A quiver is of finite type if it has only a finite number of indecomposable representations (up to isomorphism); a representation is indecomposable if it cannot be obtained as a nontrivial direct sum. By Gabriel's Theorem, a quiver is of finite type if and only if its underlying graph is a Dynkin diagram of type $A, D$ or $E$. See [45] and references therein.

\section{Et cetera}

And the list goes on: simple singularities, finite subgroups of $S U(2)$, symmetric matrices with nonnegative integer entries and eigenvalues between -2 and 2 , etc. For more, see [28, 33, 59. In Section 4.2, we will present yet another classification that is parallel to Cartan-Killing: the classification of the cluster algebras of finite type.

\subsection{Reduced words and permutohedra}

Each element $w \in W$ can be written as a product of elements of $S$ :

$$
w=s_{i_{1}} \cdots s_{i_{\ell}} .
$$

A shortest factorization of this form (or the corresponding sequence of subscripts $\left.\left(i_{1}, \ldots, i_{\ell}\right)\right)$ is called a reduced word for $w$; the number of factors $\ell$ is called the length of $w$.

Any finite Coxeter group has a unique element $w_{\circ}$ of maximal length. In the symmetric group $\mathcal{S}_{n+1}=A_{n}$, this is the permutation $w_{\circ}$ that reverses the order of the elements of the set $\{1, \ldots, n+1\}$.

Example 2.12. Let $W=\mathcal{S}_{4}$ be the Coxeter group of type $A_{3}$. The standard choice of simple reflections yields $S=\left\{s_{1}, s_{2}, s_{3}\right\}$, where $s_{1}, s_{2}$ and $s_{3}$ are the transpositions which interchange 1 with 2,2 with 3 , and 3 with 4 , respectively. (Cf. Example 1.7])

The word $s_{1} s_{2} s_{1} s_{3} s_{2} s_{3}$ is a non-reduced word for the permutation that interchanges 1 with 3 and 2 with 4 . This permutation has two reduced words $s_{2} s_{1} s_{3} s_{2}$ and $s_{2} s_{3} s_{1} s_{2}$.

An example of a reduced word for $w_{\circ}$ is $s_{1} s_{2} s_{1} s_{3} s_{2} s_{1}$. There are 16 such reduced words altogether. (Cf. Example 2.14 and Theorem 2.15.)

Recall from Section 1.2 that we label the regions $R_{w}$ of the Coxeter arrangement by the elements of the reflection group $W$, so that $R_{w}$ is the image of $R_{1}$ under the action of $w$. More generally, $R_{u v}=u\left(R_{v}\right)$.

Lemma 2.13. In the Coxeter arrangement associated with a reflection group $W$, regions $R_{u}$ and $R_{v}$ are adjacent (that is, share a codimension 1 face) if and only if $u^{-1} v$ is a simple reflection.

Thus, moving to an adjacent region is encoded by multiplying on the right by a simple reflection; cf. Figure 1.1. (Warning: this simple reflection is generally not the same as the reflection through the hyperplane separating the two adjacent regions.) Consequently, reduced words for an element $w \in W$ correspond to equivalence classes of paths from $R_{1}$ to $R_{w}$ in the ambient space of the Coxeter arrangement. More precisely, we consider the paths that cross hyperplanes of the arrangement 
one at a time, and cross each hyperplane at most once; two paths are equivalent if they cross the same hyperplanes in the same order.

In order to make the correspondence between paths and reduced words more explicit, one can restrict the paths to the edges of the $W$-permutohedron, a convex polytope that we will now define. Fix a point $x$ in the interior of $R_{1}$. The $W$ permutohedron is the convex hull of the orbit of $x$ under the action of $W$. The name "permutohedron" comes from the fact that the vertices of an $A_{n}$-permutohedron are obtained by permuting the coordinates of a generic point in $\mathbb{R}^{n+1}$.

Example 2.14. The $A_{2}, B_{2}$ and $G_{2}$ permutohedra are respectively a hexagon, an octagon and a dodecagon; under the right choices of $x$, these polygons are regular. Figures 2.3 and 2.4 show the permutohedra of types $A_{3}$ and $B_{3}$. Each of these realizations derives from a choice of $x \in R_{1}$ which makes the permutohedron an Archimedean solid, so that in particular its facets are all regular polygons. The non-crystallographic $H_{3}$-permutohedron is also an Archimedean solid].

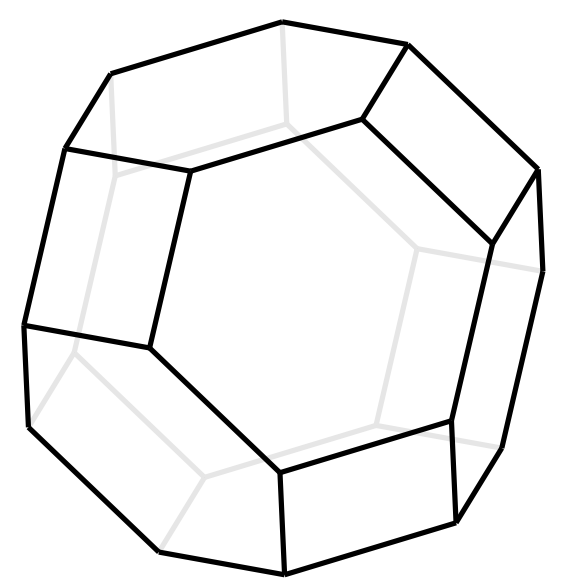

Figure 2.3. The permutohedron of type $A_{3}$

In both pictures, the bottom vertex can be associated with the identity element $1 \in W$, so that the top vertex is $w_{\circ}$. A reduced word for $w$ corresponds to a path along edges from 1 to $w$ which moves up in a monotone fashion. There are 16 such paths from 1 to $w_{\circ}$ in the $A_{3}$-permutohedron; cf. Example 2.12

The following beautiful formula is due to R. Stanley [49].

Theorem 2.15. The number of reduced words for $w_{\circ}$ in the reflection group $A_{n}$ is

$$
\frac{\left(\begin{array}{c}
n+1 \\
2
\end{array}\right) !}{1^{n} 3^{n-1} 5^{n-2} \cdots(2 n-1)^{1}} .
$$

\footnotetext{
${ }^{1}$ An Archimedean solid is a non-regular polytope whose all facets are regular polygons, and whose symmetry group acts transitively on vertices. In dimension 3, there are 13 Archimedean solids. The permutohedra of types $A_{3}, B_{3}$, and $H_{3}$ are also known as the truncated octahedron, great rhombicuboctahedron, and great rhombicosidodecahedron, respectively. See, e.g., [55.
} 


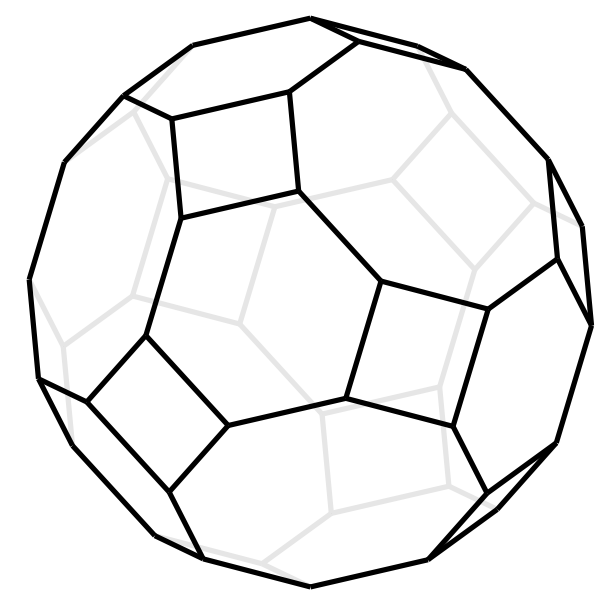

Figure 2.4. The permutohedron of type $B_{3}$

\subsection{Coxeter element and Coxeter number}

The underlying graph of the Coxeter diagram for a finite Coxeter group has no cycles. Hence it is bipartite, i.e., we can write a disjoint union $I=I_{+} \cup I_{-}$such that each of the sets $I_{+}$and $I_{-}$is totally disconnected in the Coxeter diagram. An example is shown in Figure 2.5, where the elements of $I_{+}$and $I_{-}$are marked by + and - , respectively.

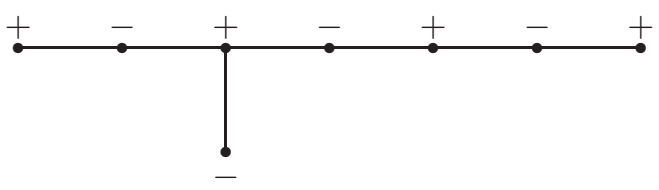

Figure 2.5. Bi-partition of the nodes of the Coxeter diagram of type $E_{8}$

The simple reflections associated with $I_{+}$(resp., $I_{-}$) commute pairwise. Consequently, the following is well-defined:

$$
c=\left(\prod_{i \in I_{+}} s_{i}\right)\left(\prod_{i \in I_{-}} s_{i}\right) .
$$

The element $c \in W$ is called the Coxeter element 2 .

Example 2.16. In type $A_{n}$, let $I_{-}$(resp., $I_{+}$) consist of the odd (resp., even) numbers in $I=[n]$. Then for example in $A_{5}=\mathcal{S}_{6}$, we have $c=s_{2} s_{4} s_{1} s_{3} s_{5}$.

Thinking of $W$ as a reflection group, the Coxeter element $c$ is an interesting orthogonal transformation. One important feature of $c$ is that it fixes a certain two-dimensional plane $L$ (as a set, not pointwise). The action of $c$ on $L$ can be analyzed to determine the order of $c$ as an element of $W$. This order is called the Coxeter number of $W$, and is denoted by $h$.

2 More broadly, one often calls the product of the elements in $S$ (in any order) a Coxeter element, but for our present purposes the definition above will do. 
Example 2.17. Figure 2.6 shows the Coxeter arrangement of type $A_{3}$ and the plane $L$ fixed by the Coxeter element $c=s_{2} s_{1} s_{3}$ (dotted). The great circles represent the intersections of the six reflecting hyperplanes with a unit hemisphere. The sphere is opaque, so only half of each circle is visible, and appears either as a half of an ellipse or as a straight line segment. (The "equator" does not represent a hyperplane in the arrangement.) The restriction of $c$ onto $L$ has order 4 , so the Coxeter number for $A_{3}$ is $h=4$.

Example 2.18. Figure 2.7 is a similar picture for $B_{3}$, illustrating that the Coxeter number for $B_{3}$ is $h=6$. In this picture, the equator does represent a hyperplane in the arrangement.

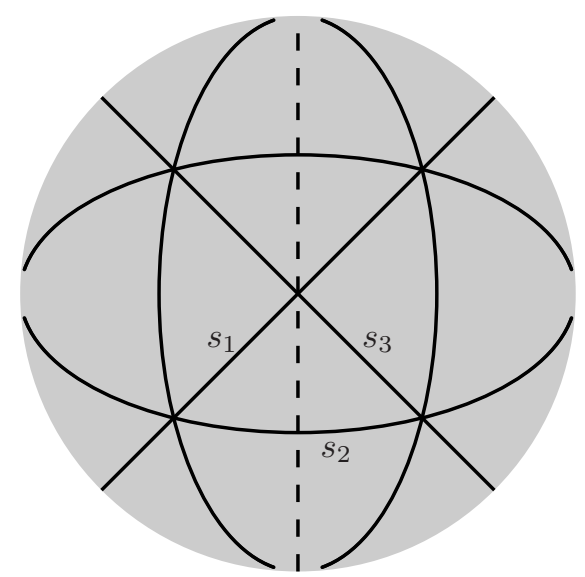

Figure 2.6. The Coxeter arrangement $A_{3}$ and the plane fixed by the Coxeter element

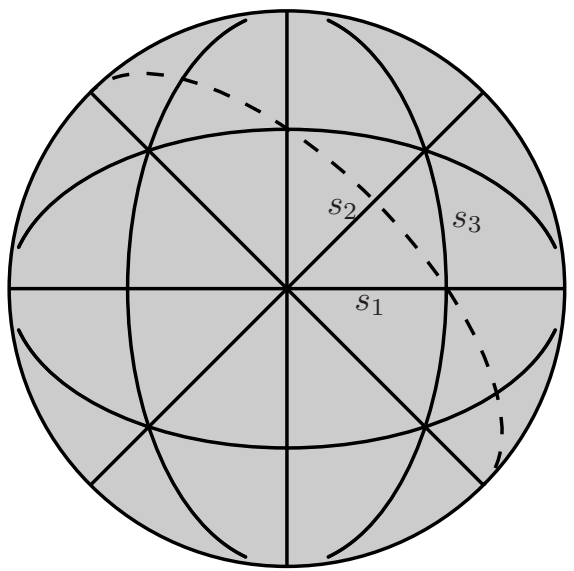

Figure 2.7. The Coxeter arrangement $B_{3}$ and the plane fixed by the Coxeter element 
The action of $c$ on $L$ also leads to a determination of its eigenvalues, which all have the form $e^{2 m i \pi / h}$, where $m$ is a positive integer less than $h$. The $n$ values of $m$ which arise in this way are called the exponents of $W$. We denote the exponents by $e_{1}, \ldots, e_{n}$. They pop up everywhere in the combinatorics of root systems and Coxeter groups. For instance, the order (i.e., cardinality) of $W$ is expressed in terms of the exponents by

$$
|W|=\prod_{i=1}^{n}\left(e_{i}+1\right) .
$$

See Section 5.1 for more examples.

For a finite irreducible Coxeter group $W$ Figure 2.8 tabulates some classical numerical invariants associated to $W$ and the corresponding (not necessarily crystallographic) root system $\Phi$.

\begin{tabular}{|c|c|c|c|c|}
\hline type of $\Phi$ & $\left|\Phi_{+}\right|$ & $h$ & $e_{1}, \ldots, e_{n}$ & $|W|$ \\
\hline \hline$A_{n}$ & $n(n+1) / 2$ & $n+1$ & $1,2, \ldots, n$ & $(n+1) !$ \\
\hline$B_{n}, C_{n}$ & $n^{2}$ & $2 n$ & $1,3,5, \ldots, 2 n-1$ & $2^{n} n !$ \\
\hline$D_{n}$ & $n(n-1)$ & $2(n-1)$ & $1,3,5, \ldots, 2 n-3, n-1$ & $2^{n-1} n !$ \\
\hline$E_{6}$ & 36 & 12 & $1,4,5,7,8,11$ & $2^{7} 3^{4} 5$ \\
\hline$E_{7}$ & 63 & 18 & $1,5,7,9,11,13,17$ & $2^{10} 3^{4} 5 \cdot 7$ \\
\hline$E_{8}$ & 120 & 30 & $1,7,11,13,17,19,23,29$ & $2^{14} 3^{5} 5^{2} 7$ \\
\hline$F_{4}$ & 24 & 12 & $1,5,7,11$ & $2^{7} 3^{2}$ \\
\hline$G_{2}$ & 6 & 6 & 1,5 & $2^{2} 3$ \\
\hline$H_{3}$ & 15 & 10 & $1,5,9$ & $2^{3} 3 \cdot 5$ \\
\hline$H_{4}$ & 60 & 30 & $1,11,19,29$ & $2^{6} 3^{2} 5^{2}$ \\
\hline$I_{2}(m)$ & $m$ & $m$ & $1, m-1$ & $2 m$ \\
\hline
\end{tabular}

Figure 2.8. Number of positive roots, Coxeter number, exponents, and the order of $W$. 


\section{LECTURE 3 Associahedra and Mutations}

\subsection{Associahedron}

We start by discussing two classical problems of combinatorial enumeration.

(i) Count the number of bracketings (parenthesizations) of a non-associative product of $n+2$ factors. Note that we need $n$ pairs of brackets in order to make the product unambiguous.

(ii) Count the number of triangulations of a convex $(n+3)$-gon by diagonals. Note that each triangulation involves exactly $n$ diagonals.

Example 3.1. In the special cases $n=1,2,3$, there are, respectively:

- 2 bracketings $(a b) c$ and $a(b c)$ of a product of 3 factors;

- 5 bracketings $((a b) c) d,(a(b c)) d, a((b c) d),(a b)(c d)$, and $a(b(c d))$ of a product of 4 factors;

- 14 bracketings of a product of 5 factors (check!).

As to triangulations, there are:

- 2 triangulations of a convex quadrilateral $(n=1)$;

- 5 triangulations of a pentagon ( $n=2$, Figure 3.3);

- 14 triangulations of a hexagon $(n=3$, Figure 3.4 $)$.

Theorem 3.2. Both bracketings and triangulations described above are enumerated by the Catalan numbers $\frac{1}{n+2}\left(\begin{array}{c}2 n+2 \\ n+1\end{array}\right)$.

There are a great many families of combinatorial objects enumerated by the Catalan numbers; more than a hundred of those are listed in [50. This list includes: ballot sequences; Young diagrams and tableaux satisfying certain restrictions; noncrossing partitions; trees of various kinds; Dyck paths; permutations avoiding patterns of length 3; and much more. In Lecture 5] we will discuss several additional members of the "Catalan family," together with their analogues for arbitrary root systems. (We will see that the ordinary Catalan numerology should be considered as "type $A . "$ )

A bijection between bracketings and triangulations is described in Figure 3.1.

For a fixed $n$, the bracketings naturally form the set of vertices of a graph whose edges correspond to applications of the associativity axiom. Figure 3.2 shows this graph for $n=2$. 


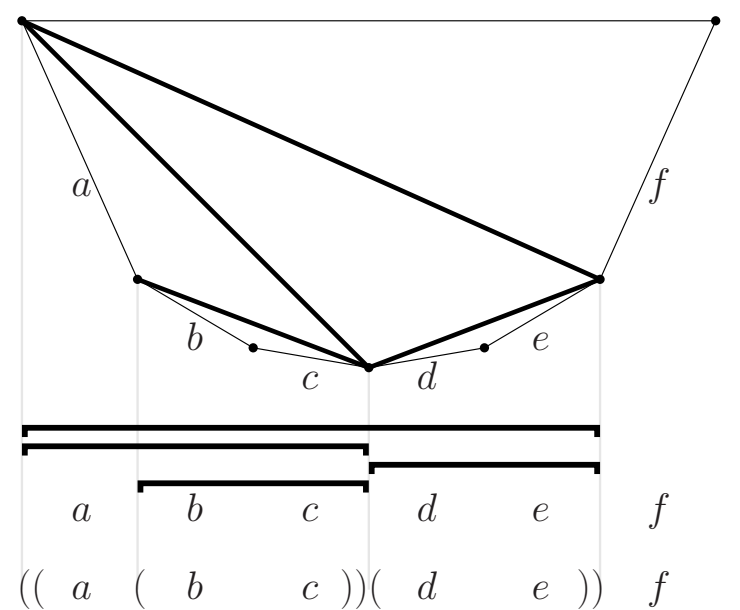

Figure 3.1. The bijection between triangulations and bracketings.

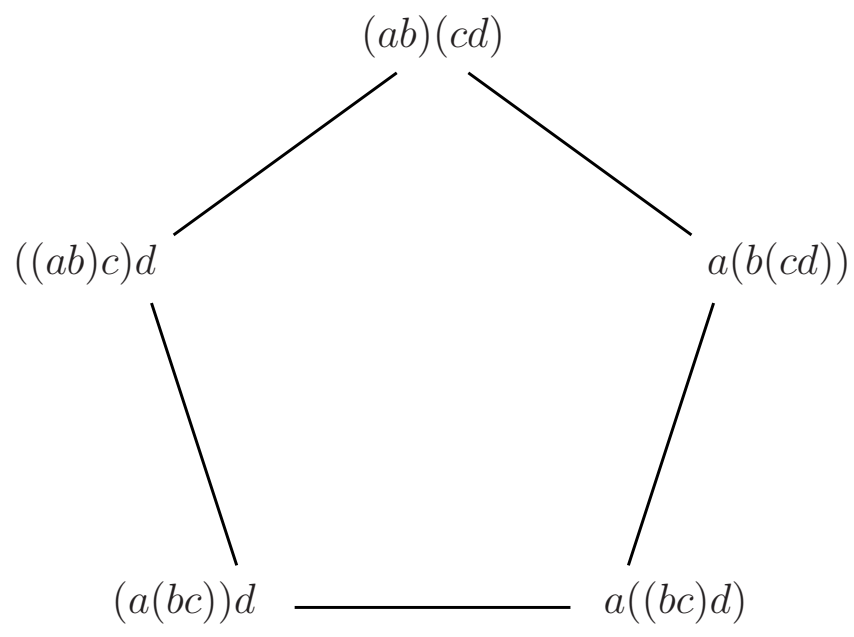

Figure 3.2. Applying associativity to the bracketings of $a b c d$.

The bijection illustrated in Figure 3.1 translates an application of the associativity axiom into a diagonal flip on the corresponding triangulation. That is, one removes a diagonal to create a quadrilateral, then replaces the removed diagonal with the other diagonal of the quadrilateral.

We call the graph defined by diagonal flips the exchange graph. The exchange graphs for $n=2$ and $n=3$ are shown in Figures 3.3 and 3.4 .

The drawing of the exchange graph in Figure 3.4 fails to convey its crucial property: this exchange graph is the 1-skeleton of a convex polytope, the 3-dimensional associahedron. (Sometimes it is also called the Stasheff polytope, after J. Stasheff, who first defined it in $\mathbf{5 2}$.) Figure 3.5 shows a polytopal realization of this associahedron. 


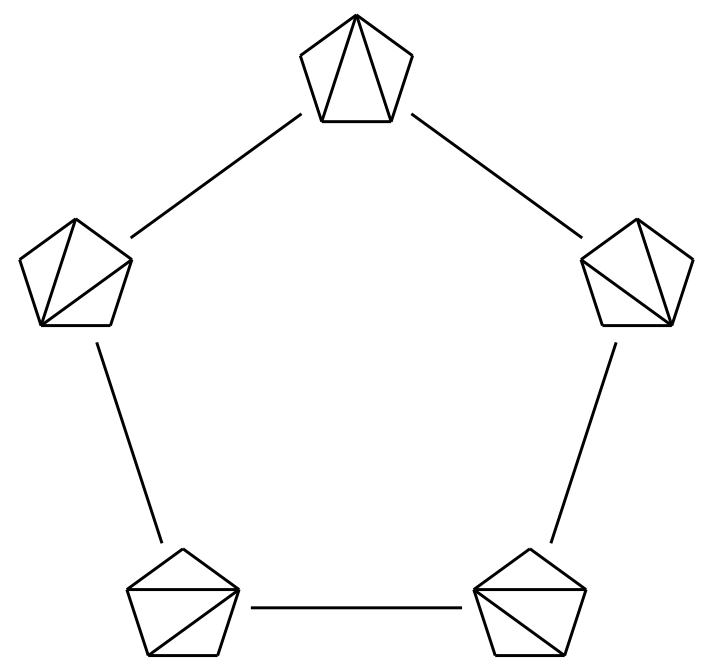

Figure 3.3. The exchange graph for triangulations of a pentagon.

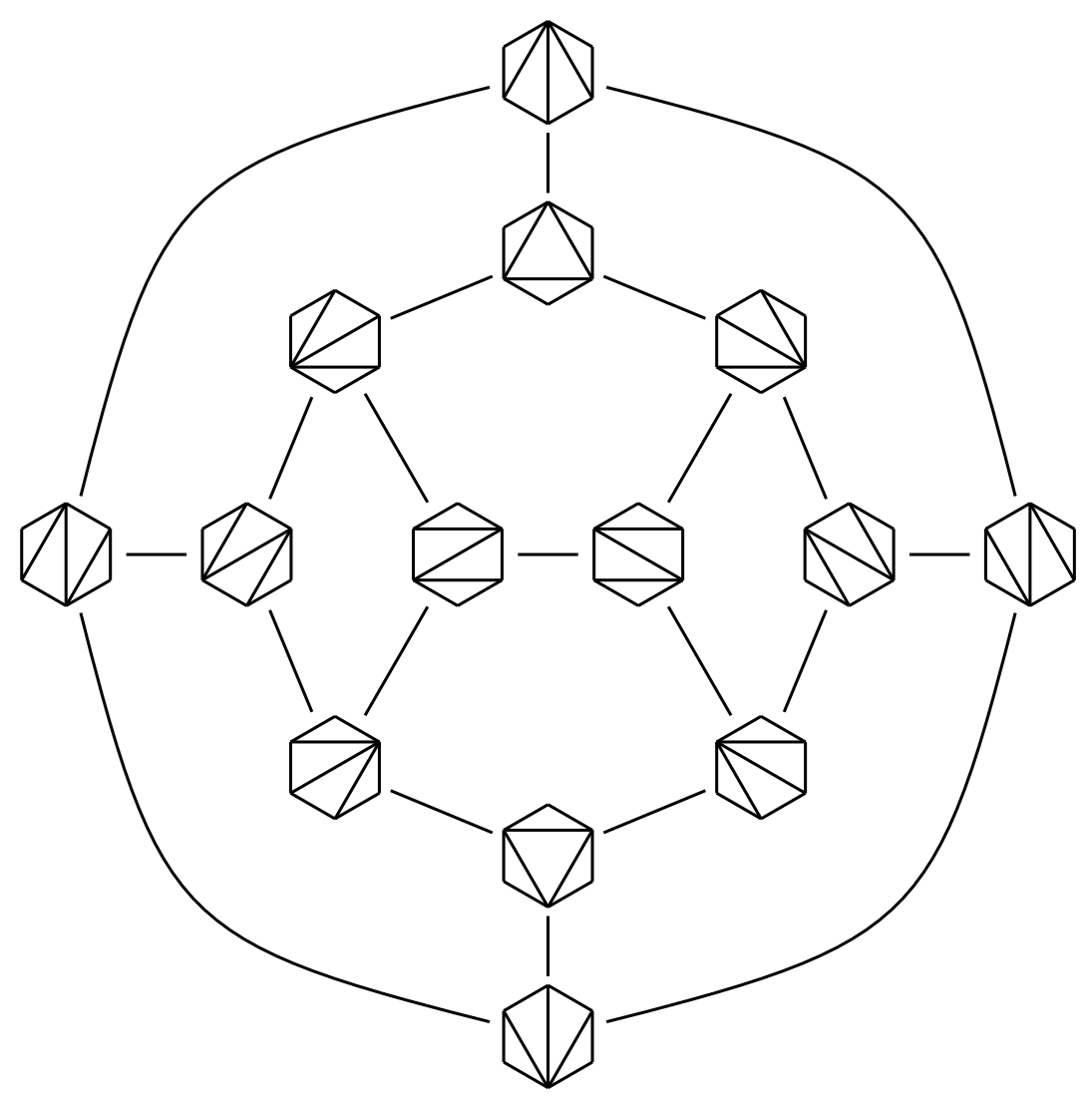

Figure 3.4. The exchange graph for triangulations of a hexagon. 


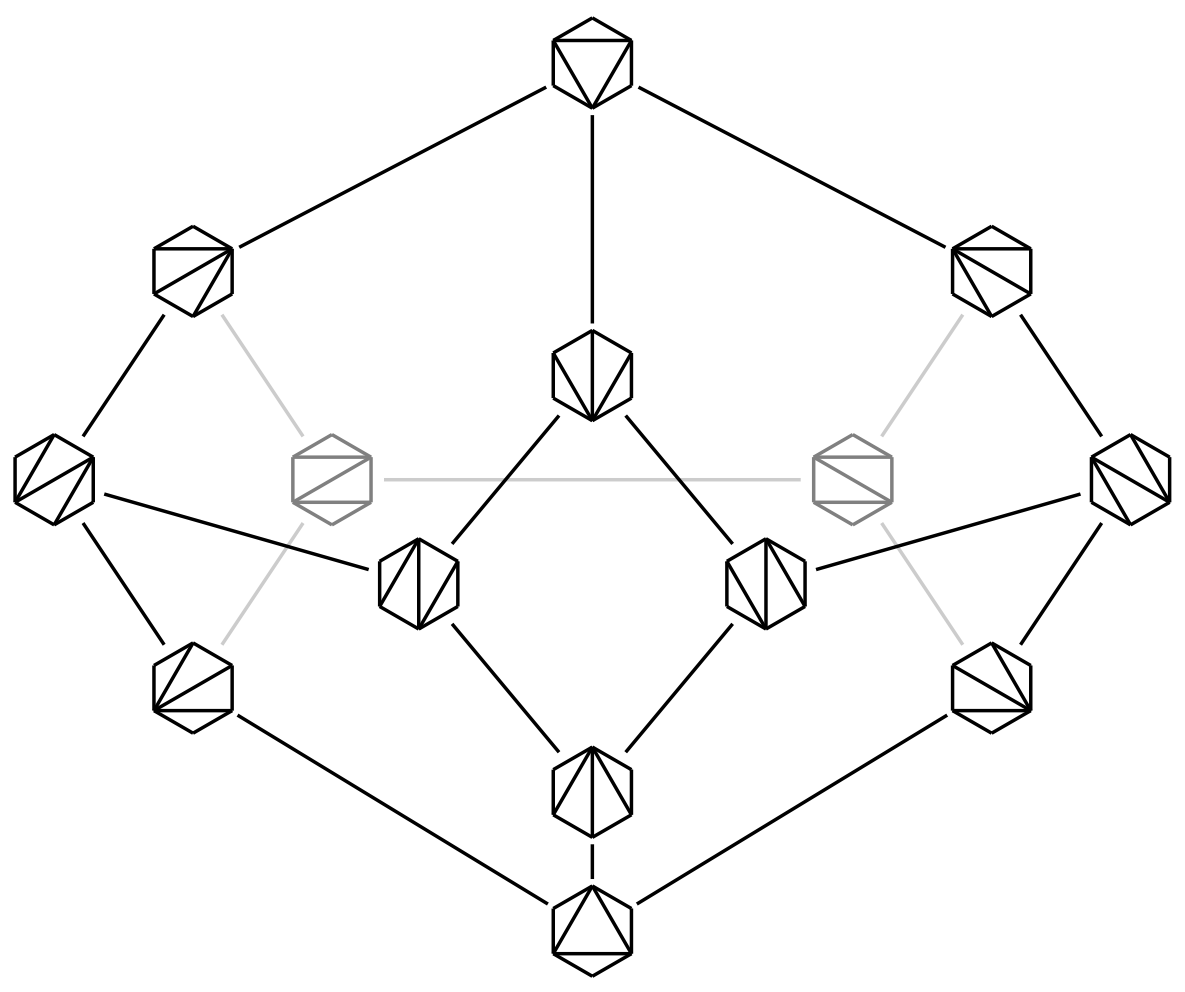

Figure 3.5. The 3-dimensional associahedron.

In order to formally define the $n$-dimensional associahedron, we start by describing the object which is dual to it, in the same sense in which the octahedron is dual to the cube, and the dodecahedron is dual to the icosahedron.

Definition 3.3 (The dual complex of an associahedron). Consider the following simplicial complex:

\begin{tabular}{|rl|}
\hline vertices: & diagonals of a convex $(n+3)$-gon \\
simplices: & $\begin{array}{l}\text { partial triangulations of the }(n+3) \text {-gon } \\
\text { (viewed as collections of non-crossing diagonals) }\end{array}$ \\
maximal simplices: & $\begin{array}{l}\text { triangulations of the }(n+3) \text {-gon } \\
\text { (collections of } n \text { non-crossing diagonals). }\end{array}$ \\
\hline
\end{tabular}

Figure 3.6 shows this simplicial complex for $n=3$, superimposed on a faint copy of the exchange graph. Note that the facial structures of the 3-dimensional associahedron and its dual complex are indeed "dual" to each other: two vertices of one polyhedron are adjacent if and only if the corresponding faces of the other polyhedron share an edge. 


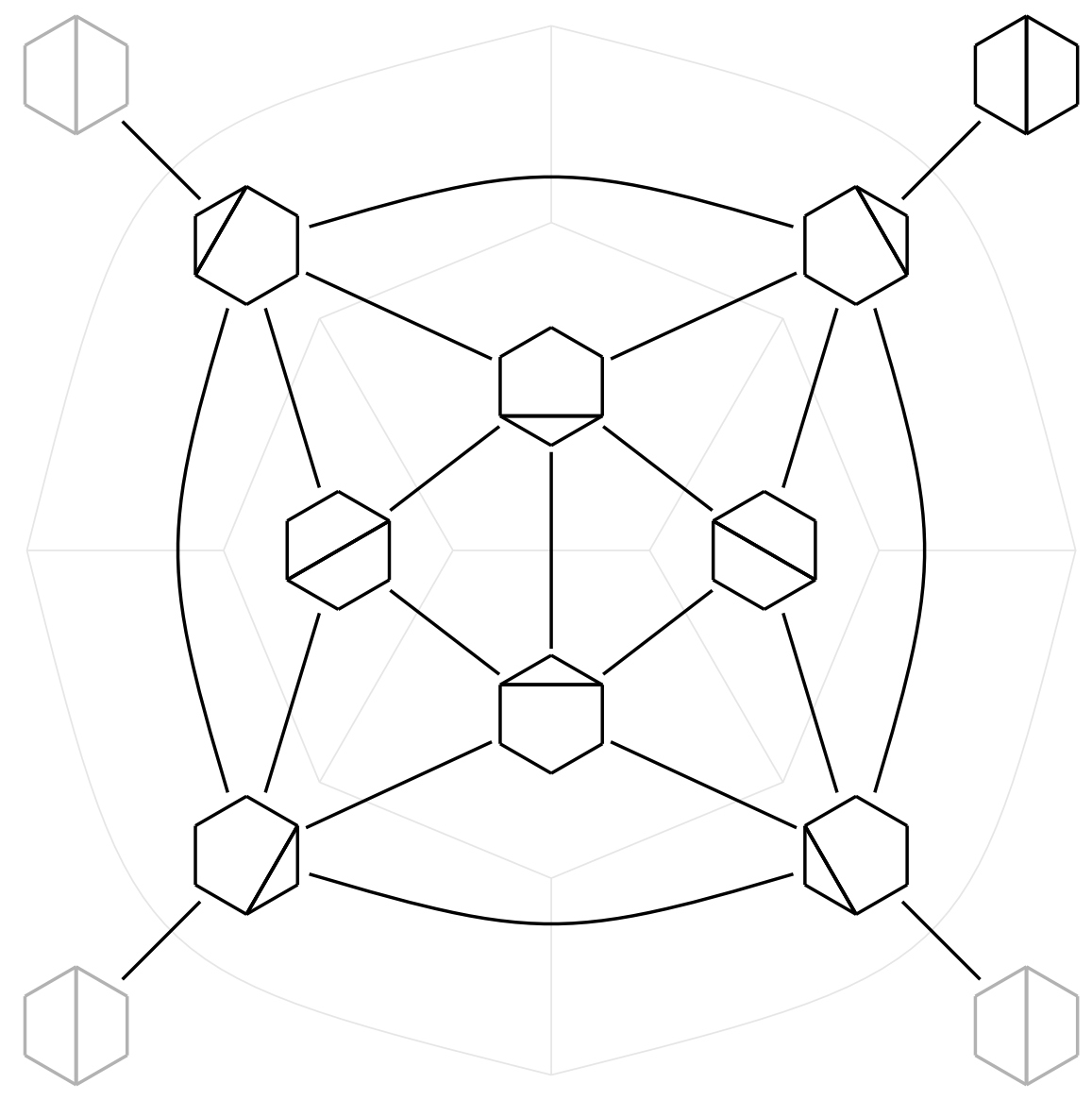

Figure 3.6. The simplicial complex dual to the 3-dimensional associahedron.

It is not clear a priori that these complexes are topological spheres. But, as already mentioned, more is true.

Theorem 3.4. The simplicial complex described in Definition 3.3 can be realized as the boundary of an n-dimensional convex polytope.

Theorem 3.4 (or its equivalent reformulations) were proved independently by J. Milnor, M. Haiman, and C. W. Lee (first published proof [36]). This theorem also follows as a special case of the very general theory of secondary polytopes developed by I. M. Gelfand, M. Kapranov and A. Zelevinsky [30].

Definition 3.5 (The associahedron). The $n$-dimensional associahedron is the convex polytope (defined up to combinatorial equivalence) that is dual (or polar, see [58, Sec. 2.3]) to the polytope of Theorem 3.4. 
The facial structure of an associahedron as a cell complex is dual to that of its polar:

\begin{tabular}{|rl|}
\hline vertices: & triangulations \\
faces: & partial triangulations \\
facets: & diagonals \\
edges: & diagonal flips \\
\hline
\end{tabular}

The labeling of the facets of an $n$-dimensional associahedron by the diagonals of an $(n+3)$-gon is illustrated in Figure 3.7 for the special case $n=3$ (compare to Figure 3.4).

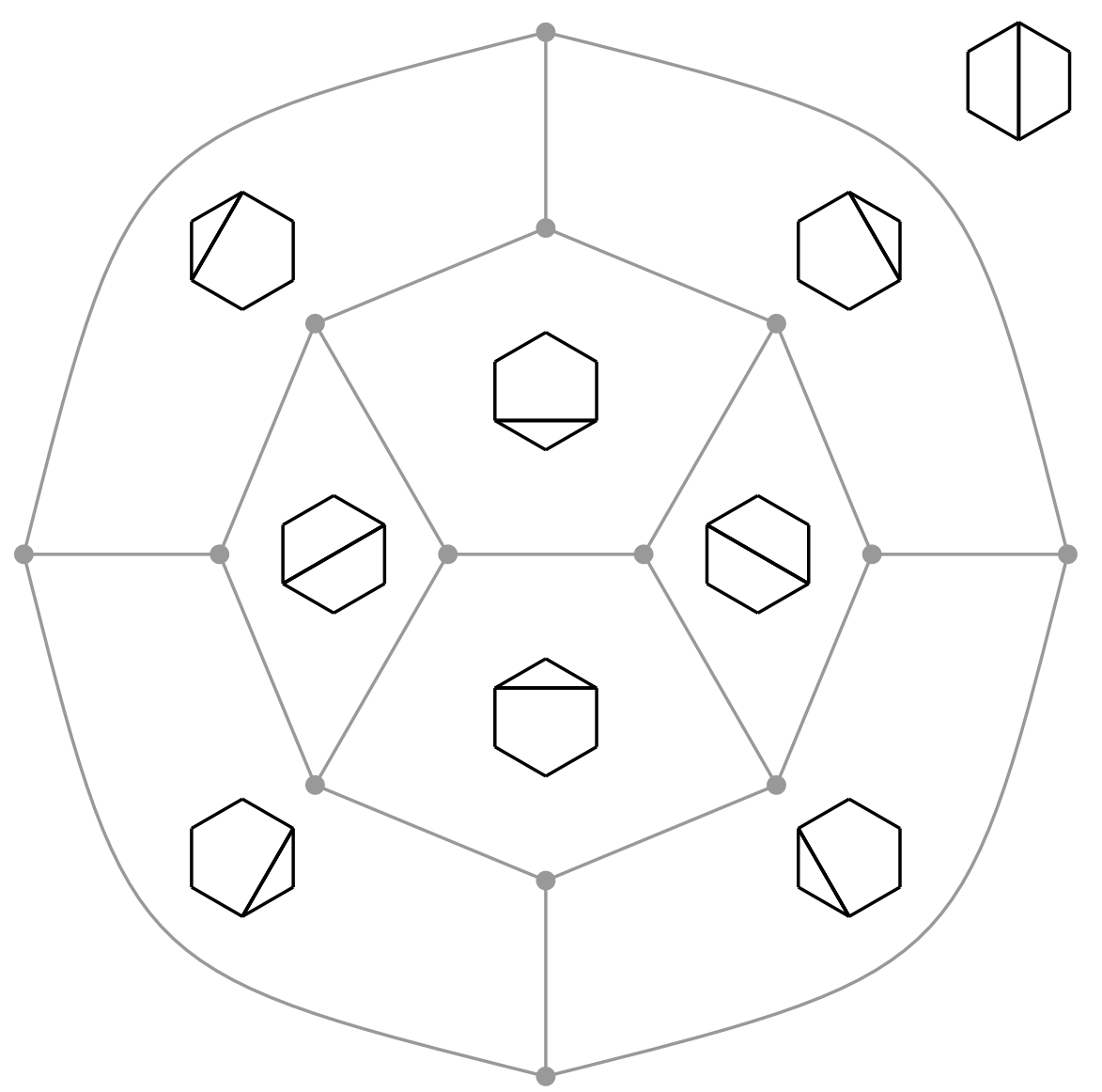

Figure 3.7. Labeling the facets of the associahedron by diagonals

We note that we could have defined the associahedron directly, as a cell complex whose cell structure is described by (6). (This would require resolving some technical issues that we would rather avoid here.) The fact that these cell complexes are polytopal-i.e., the fact that a combinatorially defined associahedron can be realized as a convex polytope — is essentially equivalent to Theorem 3.4 . 
Associahedra play an important role in homotopy theory and the study of operads [53, in the analysis of real moduli/configuration spaces [16], and other branches of mathematics. In these notes, we restrict our attention to the combinatorial aspects of the associahedra.

An $n$-dimensional polytope is called simple if every vertex is incident to exactly $n$ edges. This is the case for the associahedron, as every triangulation of an $(n+3)$ gon is adjacent to precisely $n$ others in the exchange graph.

Much is known about the facial structure and enumerative invariants of the associahedron. For example, each face is the direct product of smaller associahedra. The entries of the h-vector of the associahedron are the Narayana numbers (see Section 5.2). This allows one to calculate the number of faces of each dimension.

\subsection{Cyclohedron}

The $n$-dimensional cyclohedron (also known as the Bott-Taubes polytope [8]) is constructed similarly to the associahedron using centrally-symmetric triangulations of a regular $(2 n+2)$-gon. Each edge of the cyclohedron represents either a diagonal flip involving two diameters of the polygon, or a pair of two centrally-symmetric diagonal flips. Figures 3.8 and 3.9 show the 2- and 3-dimensional cyclohedra respectively. As these figures suggest, the cyclohedron is a convex polytope for any $n$. Explicit polytopal realizations of cyclohedra were constructed by M. Markl $\mathbf{3 8}$ and R. Simion [4]. Each face of a cyclohedron is a product of smaller cyclohedra and associahedra.

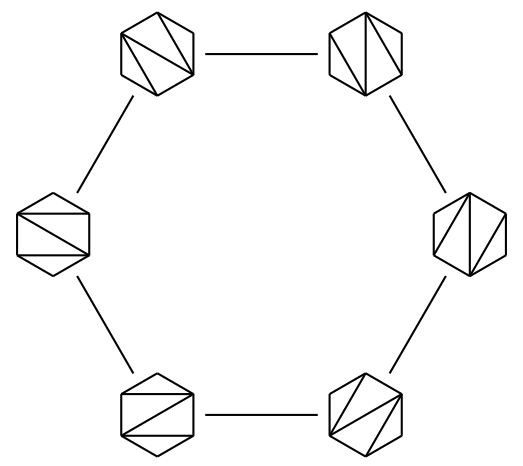

Figure 3.8. The 2-dimensional cyclohedron

Further details about the combinatorics of cyclohedra, and about their appearance in the study of configuration spaces can be found in $\mathbf{1 7}$.

The geometry of associahedra and cyclohedra is related to the geometry of permutohedra, as the following theorem (due to Tonks [54) shows.

Theorem 3.6. The 1-skeleton of the n-dimensional associahedron (resp., cyclohedron) can be obtained from the 1-skeleton of the permutohedron of type $A_{n}$ (resp., type $B_{n}$ ) by contraction of edges.

Theorem 3.6 is further discussed in Section 5.4 in connection with Theorem 5.11. For $n=3$, the theorem is illustrated in Figure 3.10. (Cf. Figures 2.3 and 2.4)

In light of Theorem 3.6, the cyclohedron can be viewed as a "type $B$ counterpart" of the associahedron (which is a "type $A$ " object). 


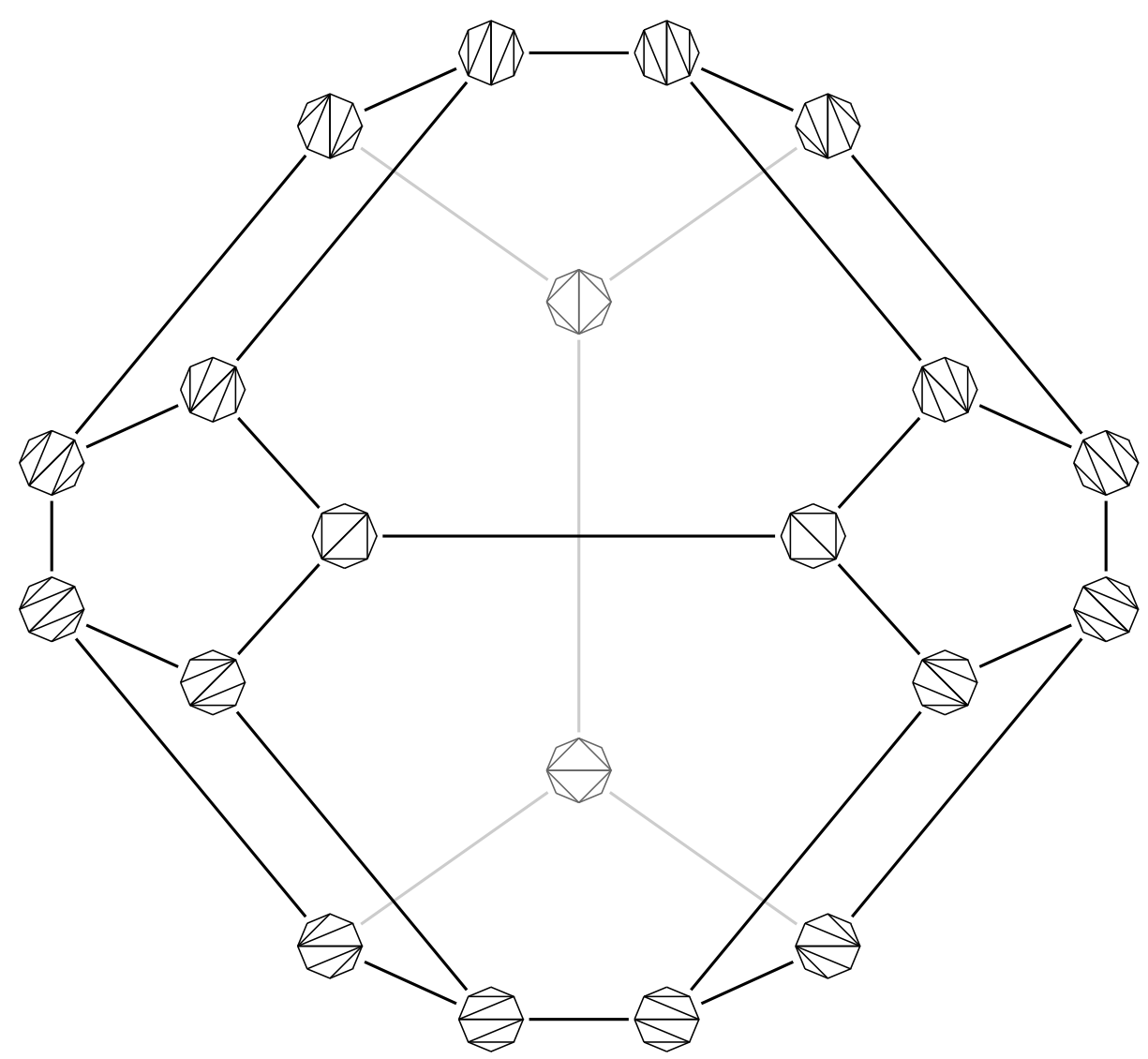

Figure 3.9. The 3-dimensional cyclohedron
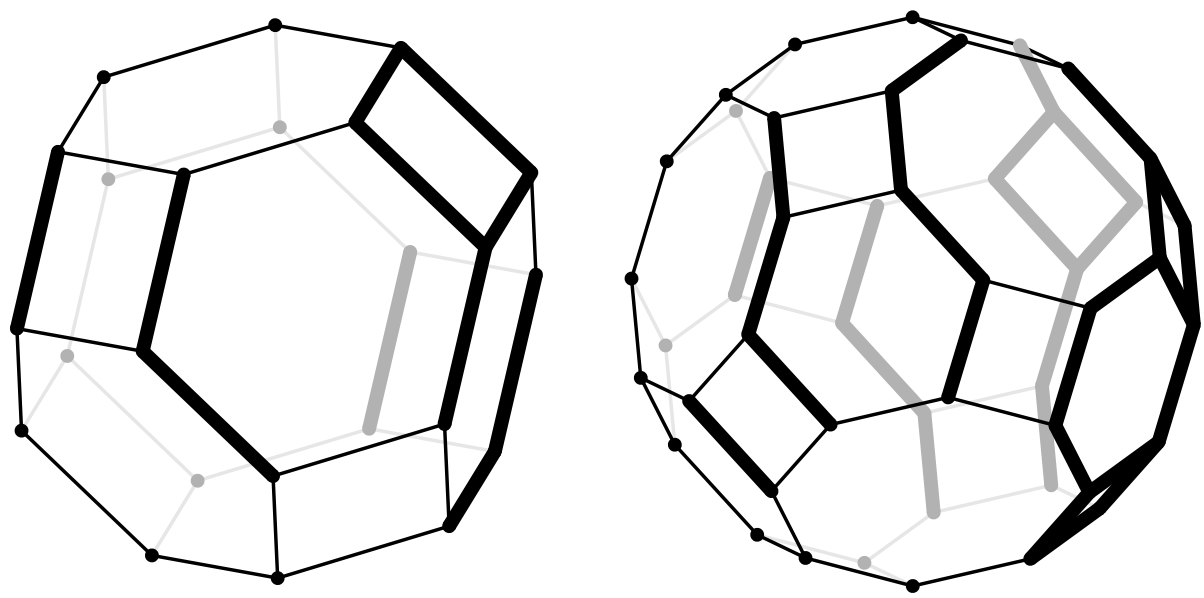

Figure 3.10. Contracting edges of permutohedra of types $A_{3}$ and $B_{3}$ yields an associahedron and a cyclohedron 


\subsection{Matrix mutations}

Having looked closely at the associahedron and the cyclohedron, one is naturally led to wonder: are these two just a pair of isolated constructions, or is there a general framework that includes them as special cases? Given that the associahedra and the cyclohedra are related to the root systems of types $A$ and $B$, respectively, is there a classification of polytopes arising within this framework that is similar to the Cartan-Killing classification of root systems?

As a first step towards answering these questions, we will develop the machinery of matrix mutations, which encode the combinatorics of various models similar to the associahedron and the cyclohedron. We begin our discussion of matrix mutations by continuing the example of the associahedron.

Fix a triangulation $T$ of the $(n+3)$-gon. Label the $n$ diagonals of $T$ arbitrarily by the numbers $1, \ldots, n$, and label the $n+3$ sides of $T$ by the numbers $n+1, \ldots, 2 n+3$. The combinatorics of $T$ can be encoded by the (signed) edgeadjacency matrix $\tilde{B}=\left(b_{i j}\right)$. This is the $(2 n+3) \times n$ matrix whose entries are given by

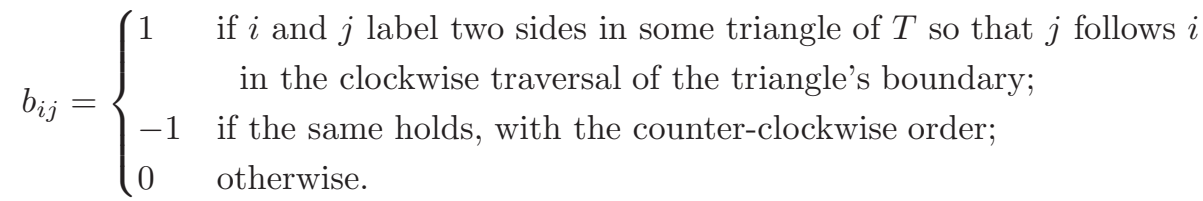

Note that the first index $i$ is a label for a side or a diagonal of the $(n+3)$-gon, while the second index $j$ must label a diagonal. The principal part of $\tilde{B}$ is an $n \times n$ submatrix $B=\left(b_{i j}\right)_{i, j \in[n]}$ that encodes the signed adjacencies between the diagonals of $T$. An example is shown in Figure 3.11 .

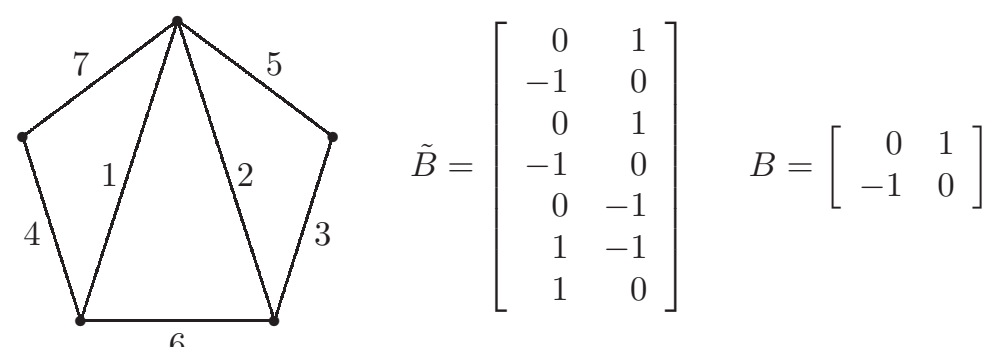

Figure 3.11. Matrices $B$ and $\tilde{B}$ for a triangulation

In the language of matrices $\tilde{B}$ and $B$, diagonal flips can be described as certain transformations called matrix mutations.

Definition 3.7. Let $B=\left(b_{i j}\right)$ and $B^{\prime}=\left(b_{i j}^{\prime}\right)$ be integer matrices. We say that $B^{\prime}$ is obtained from $B$ by a matrix mutation in direction $k$, and write $B^{\prime}=\mu_{k}(B)$, if

$$
b_{i j}^{\prime}= \begin{cases}-b_{i j} & \text { if } k \in\{i, j\} \\ b_{i j}+\left|b_{i k}\right| b_{k j} & \text { if } k \notin\{i, j\} \text { and } b_{i k} b_{k j}>0 \\ b_{i j} & \text { otherwise. }\end{cases}
$$

It is easy to check that a matrix mutation is an involution, i.e., $\mu_{k}\left(\mu_{k}(B)\right)=B$. 
Lemma 3.8. Assume that $\tilde{B}$ and $\tilde{B}^{\prime}$ (resp., $B$ and $B^{\prime}$ ) are the edge-adjacency matrices (resp., their principal parts) for two triangulations $T$ and $T^{\prime}$ obtained from each other by flipping the diagonal numbered $k$; the rest of the labels are the same in $T$ and $T^{\prime}$. Then $\tilde{B}^{\prime}=\mu_{k}(\tilde{B})$ (resp., $B^{\prime}=\mu_{k}(B)$ ).

Lemma 3.8 is illustrated in Figures 3.12 and 3.13 . Note that the numbering of diagonals used in defining the matrices $\tilde{B}$ and $B$ can change as we move along the exchange graph. For instance, the sequence of 5 flips shown in Figure 3.13 results in switching the labels of the two diagonals.

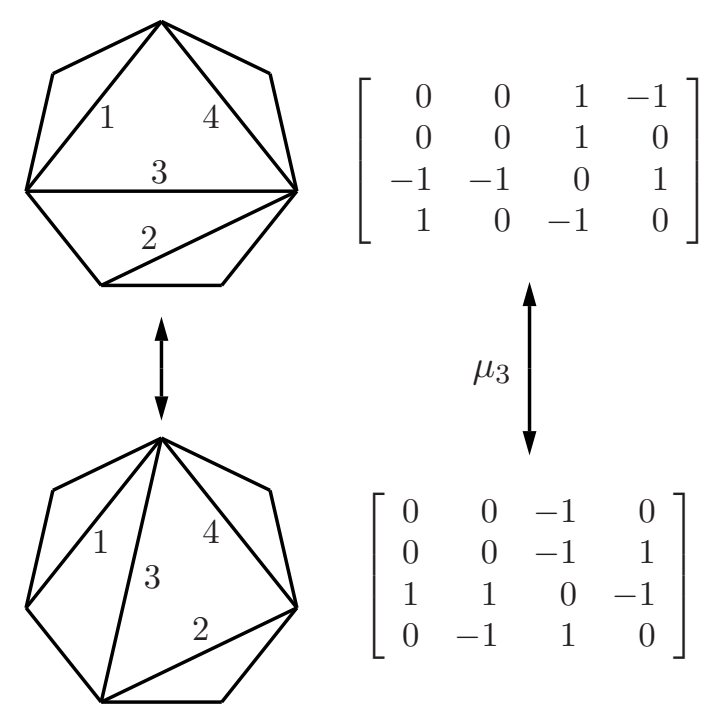

Figure 3.12. A diagonal flip and the corresponding matrix mutation

One can similarly define edge-adjacency matrices for centrally symmetric triangulations (those matrices will have entries $0, \pm 1$, and \pm 2 ), and verify that cyclohedral flips translate precisely into matrix mutations.

\subsection{Exchange relations}

We next introduce a set of algebraic (more precisely, birational) transformations that will go hand in hand with the matrix mutations. We start by explaining this construction in the case of an associahedron.

Let us fix an arbitrary initial triangulation $T_{0}$ of a convex $(n+3)$-gon, and introduce a variable for each diagonal of this triangulation, and also for each side of the $(n+3)$-gon. This gives $2 n+3$ variables altogether. We are now going to associate a rational function in these $2 n+3$ variables to every diagonal of the $(n+3)$ gon. This will be done in a recursive fashion. Whenever we perform a diagonal flip as the one shown in Figure 3.14, all but one rational functions associated to the current triangulation remain unchanged: the rational function $x$ associated with the diagonal being removed gets replaced by the rational function $x^{\prime}$ associated with the "new" diagonal, where $x^{\prime}$ is determined from the exchange relation

$$
x x^{\prime}=a c+b d .
$$



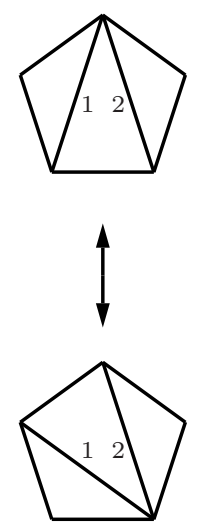

$\uparrow$

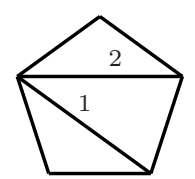

$\uparrow$
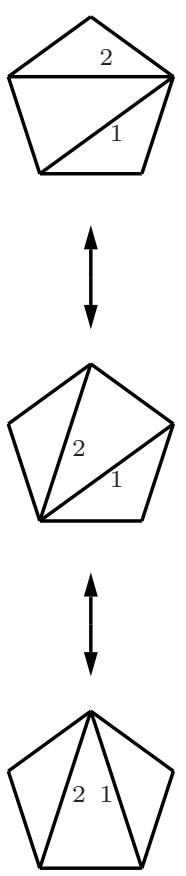

$\left[\begin{array}{rr}0 & 1 \\ -1 & 0\end{array}\right]$

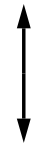

$\left[\begin{array}{rr}0 & -1 \\ 1 & 0\end{array}\right]$

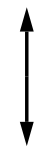

$\left[\begin{array}{rr}0 & 1 \\ -1 & 0\end{array}\right]$

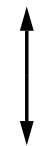

$\left[\begin{array}{rr}0 & -1 \\ 1 & 0\end{array}\right]$

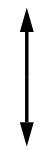

$\left[\begin{array}{rr}0 & 1 \\ -1 & 0\end{array}\right]$

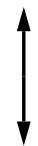

$\left[\begin{array}{rr}0 & -1 \\ 1 & 0\end{array}\right]$

Figure 3.13. Diagonal flips in a pentagon, and the corresponding matrix mutations 


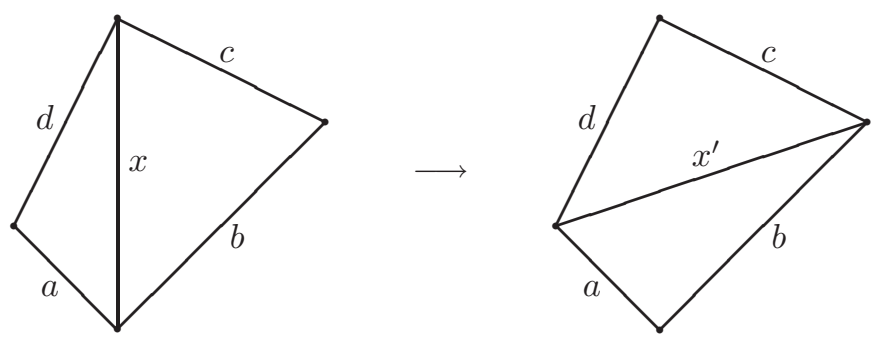

Figure 3.14. A diagonal flip

Lemma 3.9. The rational function $x_{\gamma}$ associated to each diagonal $\gamma$ does not depend on the particular choice of a sequence of flips that connects the initial triangulation with another one containing $\gamma$.

Lemma 3.9 can be rephrased as saying that there are no "monodromies" associated with sequences of flips that begin and end at the same triangulation.

To illustrate Lemma 3.9. consider the triangulations of a pentagon (i.e., $n=2$ ). We label the sides of the pentagon by the variables $q_{1}, q_{2}, q_{3}, q_{4}, q_{5}$, as shown in Figure 3.15

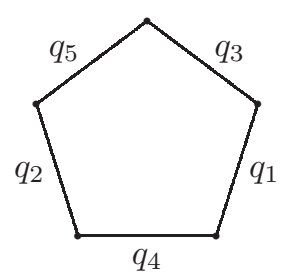

Figure 3.15. Labeling the sides of a pentagon

We then label the diagonals incident to the top vertex by the variables $y_{1}$ and $y_{2}$. Thus, our initial triangulation $T_{0}$ appears at the top of Figure 3.16. The rational functions $y_{3}, y_{4}, y_{5}$ associated with the remaining three diagonals are then computed from the exchange relations associated with the flips shown in Figure 3.16,

Starting at the top of Figure 3.16 and moving clockwise, we recursively express $y_{3}, y_{4}, \ldots$ in terms of $y_{1}, y_{2}$ and $q_{1}, \ldots, q_{5}$ :

$$
\begin{aligned}
& y_{3}=\frac{q_{2} y_{2}+q_{4} q_{5}}{y_{1}}, \\
& y_{4}=\frac{q_{3} y_{3}+q_{5} q_{1}}{y_{2}}=\frac{q_{3} q_{2} y_{2}+q_{3} q_{4} q_{5}+q_{5} q_{1} y_{1}}{y_{1} y_{2}}, \\
& y_{5}=\frac{q_{4} y_{4}+q_{1} q_{2}}{y_{3}}=\cdots=\frac{q_{3} q_{4}+q_{1} y_{1}}{y_{2}} \quad \text { (check!), }
\end{aligned}
$$

and, finally,

$$
\begin{aligned}
& y_{1}=\frac{q_{5} y_{5}+q_{2} q_{3}}{y_{4}}=\cdots=y_{1}, \\
& y_{2}=\frac{q_{1} y_{1}+q_{3} q_{4}}{y_{5}}=\cdots=y_{2},
\end{aligned}
$$




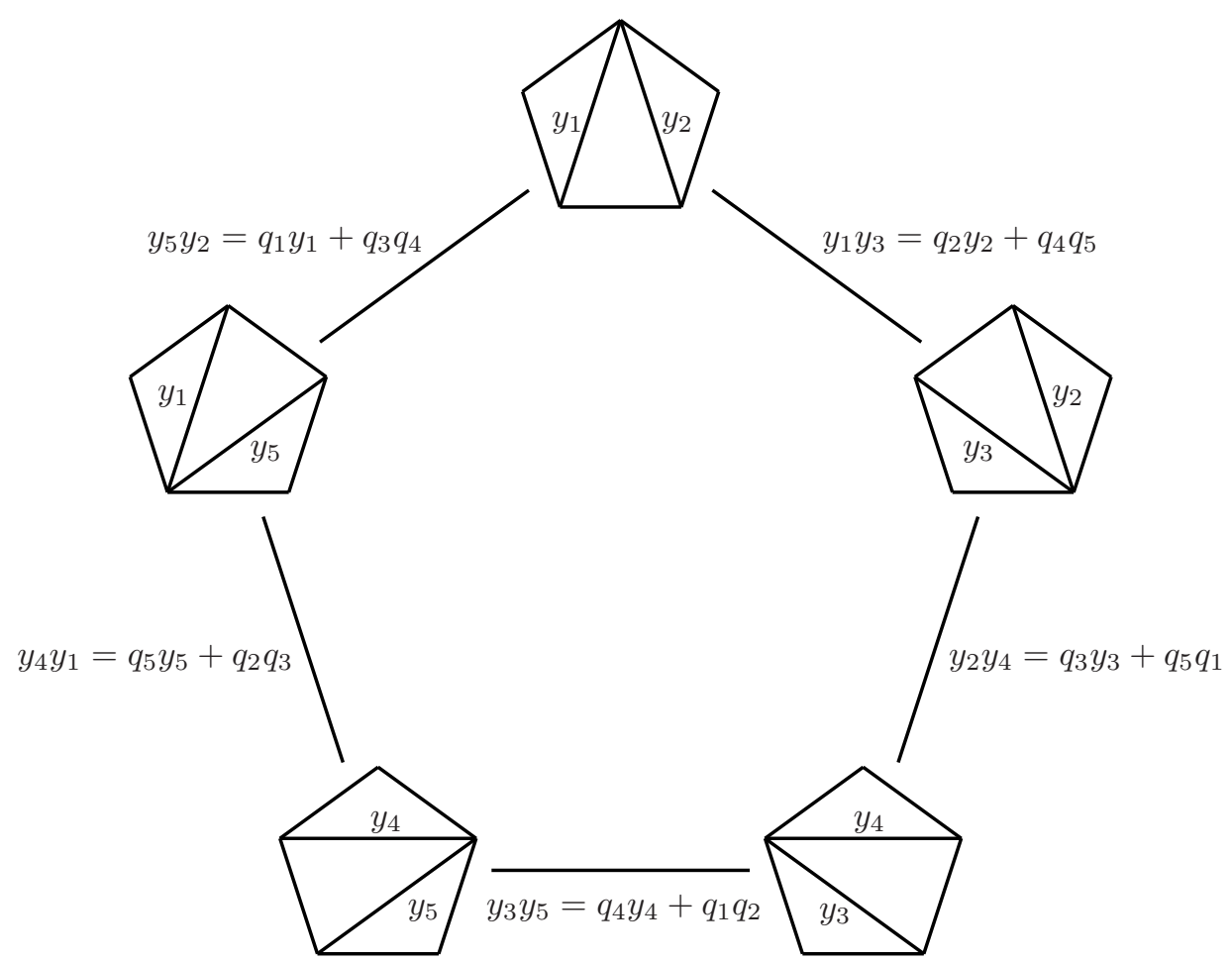

Figure 3.16. Exchange relations for the flips in a pentagon

recovering the original values and completing the cycle.

We note that under the specialization $q_{1}=\cdots=q_{5}=1$, the phenomenon we just observed is nothing else but the 5 -periodicity of the pentagon recurrence, which we have thus generalized.

Lemma 3.9 is a special case of a much more general result from the theory of cluster algebras. It can also be proved directly in at least two different ways briefly sketched below; these proofs point at connections of this subject to other areas of mathematics.

\section{Ptolemy's Theorem and hyperbolic geometry}

The classical Ptolemy's Theorem asserts that in an inscribed quadrilateral, the sum of the products of the two pairs of opposite sides equals the product of the two diagonals. This relation looks exactly like the exchange relation (8). It suggests that one can prove Lemma 3.9 simply by interpreting the rational function associated with each side or diagonal as the Euclidean length of the corresponding segment. There is however a problem with this type of argument: the space of inscribed $(n+3)$-gons (up to congruence) is $(n+3)$-dimensional, whereas we need $2 n+3$ independent variables in our setup.

The problem can be resolved by passing from Euclidean to hyperbolic geometry, where an analogue of Ptolemy's Theorem holds, and where one can "cook up" the required additional degrees of freedom. For much more on this topic, see [24, 29. 
Plücker coordinates on the Grassmannian $\operatorname{Gr}(2, n+3)$

Take a $2 \times(n+3)$ matrix $z=\left(z_{i j}\right)$. For any $k, l \in[n+3], k<l$, let us denote by

$$
P_{k l}=\operatorname{det}\left(\begin{array}{ll}
z_{1 k} & z_{1 l} \\
z_{2 k} & z_{2 l}
\end{array}\right)
$$

the $2 \times 2$ minor of $z$ that occupies columns $k$ and $l$. These minors are the homogeneous Plücker coordinates of the row span of $z$ as an element of the Grassmannian $\operatorname{Gr}(2, n+3)$ of all 2-dimensional subspaces of an $(n+3)$-space. See, e.g., [25].

It is easy to check (the special case of) the Grassmann-Plücker relations:

$$
P_{i k} P_{j l}=P_{i j} P_{k l}+P_{i l} P_{j k} .
$$

Once again, one recognizes the exchange relation (8). It is straightforward to construct, for a particular special choice of initial triangulation $T_{\circ}$, a matrix $z$ for which the values of the minors $P_{k l}$ corresponding to the sides and diagonals of $T_{\circ}$ are equal to the variables associated with these segments. It then follows by induction that the rational function associated to every diagonal is equal to the corresponding minor $P_{k l}$, implying Lemma 3.9 , 


\section{LECTURE 4 Cluster Algebras}

Our next task is to create a general axiomatic theory of mutations ("flips") and exchanges, using the above examples as prototypes. This will lead us to the basic notions and results of the theory of cluster algebras. Cluster algebras were introduced in 20] as a combinatorial/algebraic framework for the study of dual canonical bases and related total positivity phenomena. They since found applications in higher Teichmüller theory and representation theory of quivers, among other fields. All these motivations and applications will remain behind the scenes in these lectures.

Most of this lecture is based on [19, 20, 21. Sections 4.4 and 4.5 are based on 13 and 4,23 , respectively.

\subsection{Seeds and clusters}

Consider a diagonal flip that transforms a triangulation $T$ of a convex $(n+3)$-gon into another triangulation $T^{\prime}$, as shown in Figure 3.14. The corresponding exchange relation (8) can be written entirely in terms of the edge-adjacency matrix $\tilde{B}$. To be more precise, let us assume, as before, that the diagonals of $T$ have been labeled in some way by the numbers $1, \ldots, n$, whereas the sides of the $(n+3)$-gon have been assigned the labels going from $n+1$ through $m=2 n+3$. The labeling for $T^{\prime}$ is the same except for the one diagonal (say, labeled $k$ ) that is getting exchanged between $T$ and $T^{\prime}$.

This labeling of sides and diagonals of $T$ allows us to (temporarily) denote the associated rational functions by $x_{1}, \ldots, x_{m}$. For $T^{\prime}$, we get the same rational functions except that $x_{k}$ is replaced by $x_{k}^{\prime}$. Then the exchange relation under consideration takes the form

$$
x_{k} x_{k}^{\prime}=\prod_{\substack{b_{i k}>0 \\ 1 \leq i \leq m}} x_{i}^{b_{i k}}+\prod_{\substack{b_{i k}<0 \\ 1 \leq i \leq m}} x_{i}^{-b_{i k}} .
$$

In other words, the right-hand side of (9) is the sum of two monomials whose exponents are the absolute values of the entries in the $k$ th column of $\tilde{B}$, while the sign of an entry determines which monomial the corresponding term contributes to.

Example 4.1. Let $T$ be the triangulation of a pentagon in Figure 3.11 with its edges labeled $1, \ldots, 7$ as shown. The exchange relations corresponding to flipping 
the diagonals 1 and 2 are, respectively:

$$
\begin{aligned}
& x_{1} x_{1}^{\prime}=x_{6} x_{7}+x_{2} x_{4}, \\
& x_{2} x_{2}^{\prime}=x_{1} x_{3}+x_{5} x_{6},
\end{aligned}
$$

in agreement with (9).

To summarize, both the combinatorics of flips and the algebra of exchange relations can be encoded entirely in terms of the matrices $\tilde{B}$ using, first, the machinery of matrix mutations and, second, the "birational dynamics" governed by (9). We shall now use this observation to lay out the axioms of a cluster algebra. The formulation of these axioms will require some technical preparation, which hopefully will make sense to the reader in light of the examples discussed above.

A cluster algebra $\mathcal{A}$ is a commutative ring contained in an ambient field $\mathcal{F}$ isomorphic to the field of rational functions in $m$ variables over $\mathbb{Q}$. (Think of the rational functions in the variables associated with the sides and diagonals of a fixed initial triangulation.)

$\mathcal{A}$ is generated inside $\mathcal{F}$ by a (possibly infinite) set of generators. These generators are obtained from an initial seed via an iterative process of seed mutations which follows a set of canonical rules.

A seed in $\mathcal{F}$ is a pair $(\tilde{\mathbf{x}}, \tilde{B})$, where

- $\tilde{\mathbf{x}}=\left\{x_{1}, \ldots, x_{m}\right\}$ is a set 11 of $m$ algebraically independent generators of $\mathcal{F}$, which is split into a disjoint union of an $n$-element cluster $\mathbf{x}=\left\{x_{1}, \ldots, x_{n}\right\}$ and an $(m-n)$-element set of frozen variables $\mathbf{c}=\left\{x_{n+1}, \ldots, x_{m}\right\}$;

- $\tilde{B}=\left(b_{i j}\right)$ is an $m \times n$ integer matrix of rank $n$ whose principal part $B=$ $\left(b_{i j}\right)_{i, j \in[n]}$ is skew-symmetrizable, i.e., there exists a diagonal matrix $D$ with positive diagonal entries such that $D B D^{-1}$ is skew-symmetric.

(Equivalently, there exist positive integers $d_{1}, \ldots, d_{n}$ such that $d_{i} b_{i j}=-d_{j} b_{j i}$ for all $i$ and $j$.) The matrix $B$ is called the exchange matrix of a seed.

A seed mutation $\mu_{k}$ in direction $k \in\{1, \ldots, n\}$ transforms a seed $(\tilde{\mathbf{x}}, \tilde{B})$ into another seed $\left(\tilde{\mathbf{x}}^{\prime}, \tilde{B}^{\prime}\right)$ defined as follows:

- $\tilde{\mathbf{x}}^{\prime}=\tilde{\mathbf{x}}-\left\{x_{k}\right\} \cup\left\{x_{k}^{\prime}\right\}$, where $x_{k}^{\prime}$ is found from the exchange relation (9);

- $\tilde{B}^{\prime}=\mu_{k}(\tilde{B})$, i.e., $\tilde{B}$ undergoes a matrix mutation (hence so does $B$ ).

The following lemma justifies the definition of a seed mutation by showing that $\left(\tilde{\mathbf{x}}^{\prime}, \tilde{B}^{\prime}\right)$ is indeed a seed.

Lemma 4.2. Matrix mutations preserve the rank of a matrix. If $B$ is skewsymmetrizable, then so is $\mu_{k}(B)$, with the same skew-symmetrizing matrix $D$.

Note that seed mutations do not change the frozen variables $\mathbf{c}=\left\{x_{n+1}, \ldots, x_{m}\right\}$.

Example 4.3. Let $\mathbf{x}$ and $\mathbf{c}$ be the sets of variables associated with the diagonals and sides, respectively, of some triangulation of a convex $(n+3)$-gon. (Thus $m=2 n+3$.) Let $\tilde{B}$ be the sign-adjacency matrix of the triangulation. The mutations of seeds $(\tilde{\mathbf{x}}, \tilde{B})$ of this kind correspond to combining the exchange relations (8) with the matrix mutations associated with diagonal flips.

${ }^{1}$ A diligent reader might object that we call $\tilde{\mathbf{x}}$ a set rather than a sequence. This is because we regard any two seeds obtained from each other by simultaneous relabeling of the elements $x_{i}$ and the matrix entries $b_{i j}$ as identical. That is, for any permutation $w \in \mathcal{S}_{m}$ such that $w(i)=i$ for $i>n$, we make no distinction between the seeds $(\tilde{\mathbf{x}}, \tilde{B})$ and $(w(\tilde{\mathbf{x}}), w(\tilde{B}))$, where $w(\tilde{\mathbf{x}})=$ $\left(x_{w(1)}, \ldots, x_{w(m)}\right)$ and $w(\tilde{B})=\left(b_{w(i), w(j)}\right)$. 
Seed mutations generate the mutation equivalence relation on seeds: $(\tilde{\mathbf{x}}, \tilde{B}) \sim$ $\left(\tilde{\mathbf{x}}^{\prime}, \tilde{B}^{\prime}\right)$. Let $\mathcal{S}$ be an equivalence class for this relation. Thus, $\mathcal{S}$ is obtained by repeated mutations of an arbitrary initial seed in all possible directions. This creates an exchange graph. See Figure 4.1.

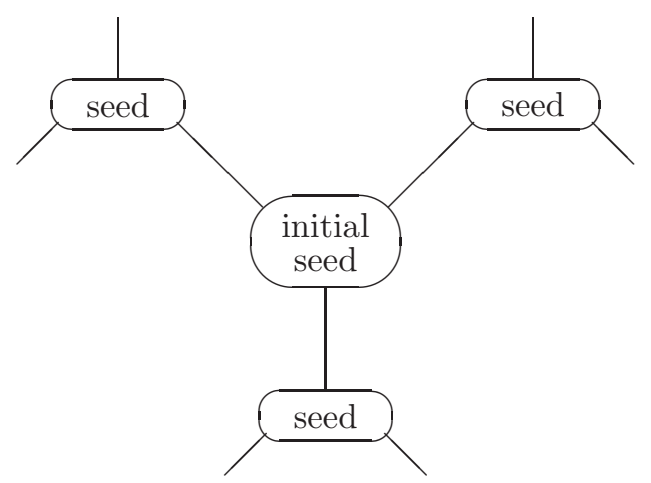

Figure 4.1. Seed mutations and the exchange graph

Let $\mathcal{X}=\mathcal{X}(\mathcal{S})$ be the union of all clusters for all the seeds in $\mathcal{S}$. The elements of $\mathcal{X}$ are called cluster variables. See Figure 4.2.

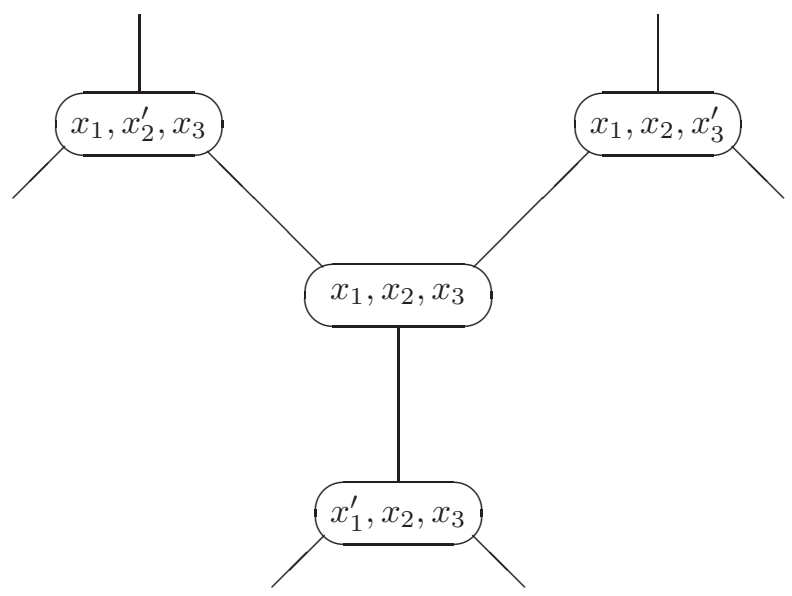

Figure 4.2. Cluster variables

The cluster algebrd $2 \mathcal{A}=\mathcal{A}(\mathcal{S})$ associated with $\mathcal{S}$ is generated inside $\mathcal{F}$ by the cluster variables in $\mathcal{X}$ together with the frozen variables $x_{n+1}, \ldots, x_{m}$ and their inverses. (A variation of this definition includes cluster and frozen variables, but none of their inverses, in the generating set.) The integer $n$ is called the rank of $\mathcal{A}$.

Theorem 4.4 (The Laurent phenomenon [20]). Any cluster variable is expressed in terms of the variables $x_{1}, \ldots, x_{m}$ of any given seed as a Laurent polynomial with integer coefficients.

2 Strictly speaking, this is a definition of a skew-symmetrizable cluster algebra of geometric type. 
Conjecture 4.5 (Nonnegativity conjecture [20]). Every coefficient in these Laurent polynomials is nonnegative.

Conjecture 4.5 has been proved in a number of special cases, including our main motivating example of an associahedron, to which we return in Example 4.6 .

Example 4.6. In the case of Example 4.3, the exchange graph on seeds is precisely the exchange graph on triangulations illustrated in Figures 3.3 and 3.4. The cluster algebra in this example is generated inside the ring of rational functions in $2 n+3$ independent variables by the rational functions associated with all diagonals and sides of the $(n+3)$-gon. (Cf. Lemma 3.9.) Here we use a variation of the definition of a cluster algebra where the inverses of frozen variables are not included in the set of generators.

This cluster algebra is canonically isomorphic to the homogeneous coordinate ring of the Grassmannian $\operatorname{Gr}(2, n+3)$ with respect to its Plücker embedding. The cluster variables, together with the frozen variables, form the set of all Plücker coordinates on this Grassmannian. Theorem 4.4 and Conjecture 4.5 (proven in this special case) assert that any Plücker coordinate is written in terms of the Plücker coordinates associated with a given triangulation as a Laurent polynomial with nonnegative integer coefficients.

\subsection{Finite type classification}

All results in this section were obtained in [21.

A cluster algebra is said to be of finite type if it has finitely many distinct seeds. Amazingly, the classification of the cluster algebras of finite type turns out to be completely parallel to the Cartan-Killing classification of (finite crystallographic) root systems. Thus there is a cluster algebra of finite type for each Dynkin diagram, or each Cartan matrix of finite type. We shall now explain how.

For a Cartan matrix $A=\left(a_{i j}\right)$ of finite type, we define a skew-symmetrizable matrix $B(A)=\left(b_{i j}\right)$ by

$$
b_{i j}=\left\{\begin{array}{cl}
0 & \text { if } i=j \\
a_{i j} & \text { if } i \neq j \text { and } i \in I_{+} ; \\
-a_{i j} & \text { if } i \neq j \text { and } i \in I_{-},
\end{array}\right.
$$

where $I_{+}$and $I_{-}$are defined as in Section 2.5. To illustrate, in type $B_{4}$, we have (cf. Example 2.3):

$$
A=\left[\begin{array}{rrrr}
2 & -2 & 0 & 0 \\
-1 & 2 & -1 & 0 \\
0 & -1 & 2 & -1 \\
0 & 0 & -1 & 2
\end{array}\right], \quad B(A)=\left[\begin{array}{rrrr}
0 & -2 & 0 & 0 \\
1 & 0 & 1 & 0 \\
0 & -1 & 0 & -1 \\
0 & 0 & 1 & 0
\end{array}\right]
$$

under the convention $I_{+}=\{1,3\}, I_{-}=\{2,4\}$.

Theorem 4.7 (Finite type classification). A cluster algebra $\mathcal{A}$ is of finite type if and only if the exchange matrix at some seed of $\mathcal{A}$ is of the form $B(A)$, where $A$ is a Cartan matrix of finite type.

The type of $A$ (in the Cartan-Killing nomenclature) is uniquely determined by the cluster algebra $\mathcal{A}$, and is called the "cluster type" of $\mathcal{A}$. 
We note that in deciding whether a cluster algebra is of finite type, the bottom part of the matrix $\tilde{B}$ plays no role whatsoever: everything is determined by its principal part $B$.

In the special cases where a cluster algebra has rank $n=2$, is of finite type (that is, one of the types $A_{2}, B_{2}$, and $G_{2}$ ), and has no frozen variables (that is, $m=2$ ), Theorem 4.7 brings us back to the recurrences of Section 1.1. Indeed, these recurrences are precisely given by the exchange relations in those cluster algebras. The periodicity of the corresponding sequences is simply a reformulation of the "finite type" property for cluster algebras.

Theorem 4.8 (Combinatorial criterion for finite type). A cluster algebra $\mathcal{A}$ is of finite type if and only if the exchange matrix $B=\left(b_{i j}\right)$ for any seed of $\mathcal{A}$ satisfies the inequalities $\left|b_{i j} b_{j i}\right| \leq 3$ for all $i, j \in\{1, \ldots, n\}$.

To rephrase, a mutation equivalence class of skew-symmetrizable $n \times n$ matrices defines a class of cluster algebras of finite type if and only if, for each matrix $B=\left(b_{i j}\right)$ in this equivalence class, the inequality $\left|b_{i j} b_{j i}\right| \leq 3$ holds for all $i$ and $j$.

Combining Theorems 4.8 and 2.4 yields the following completely elementary statement about integer matrices, no direct proof of which is known $3^{3}$.

Corollary 4.9. Let $\mathfrak{B}$ be a mutation equivalence class of skew-symmetrizable integer matrices, with the skew-symmetrizing matrix D. (Cf. Lemma 4.2.) The following are equivalent:

- any matrix $B=\left(b_{i j}\right) \in \mathfrak{B}$ satisfies the inequalities $\left|b_{i j} b_{j i}\right| \leq 3$, for all $i$ and $j$;

- there exists a matrix $B=\left(b_{i j}\right) \in \mathfrak{B}$ with the following property. Define $A=\left(a_{i j}\right)$ by

$$
a_{i j}=\left\{\begin{array}{cc}
-\left|b_{i j}\right| & \text { if } i \neq j \\
2 & \text { if } i=j .
\end{array}\right.
$$

Then $D A D^{-1}$ is positive definite.

Let $\Phi$ be an irreducible finite root system with Cartan matrix $A$, and let $\mathcal{A}$ be a cluster algebra of the corresponding cluster type. Theorem 4.7 tells us that the set $\mathcal{X}$ of cluster variables is finite. A more detailed description of this set is provided by Theorem 4.10 below.

Let $\alpha_{1}, \ldots, \alpha_{n}$ be the simple roots of $\Phi$, and let $\left\{x_{1}, \ldots, x_{n}\right\}$ be the cluster at a seed in $\mathcal{A}$ with the exchange matrix $B(A)$.

Let $\Phi_{>-1}$ denote the set of roots in $\Phi$ which are either negative simple or positive. Theorem 4.10 shows that the cluster variables in $\mathcal{A}$ are naturally labeled by the roots in $\Phi_{\geq-1}$.

Theorem 4.10. For any root $\alpha=c_{1} \alpha_{1}+\cdots+c_{n} \alpha_{n} \in \Phi_{\geq-1}$, there is a unique cluster variable $x[\alpha]$ given by

$$
x[\alpha]=\frac{P_{\alpha}\left(x_{1}, \ldots, x_{m}\right)}{x_{1}^{c_{1}} \cdots x_{n}^{c_{n}}},
$$

where $P_{\alpha}$ is a polynomial in $x_{1}, \ldots, x_{m}$ with nonzero constant term. The map $\alpha \mapsto x[\alpha]$ is a bijection between $\Phi_{\geq-1}$ and $\mathcal{X}$.

\footnotetext{
${ }^{3}$ Note added in revision. According to A. Zelevinsky, such a proof has been recently found in his
} joint work with M. Barot and C. Geiss. 
Note that the right-hand side of (10) is a Laurent polynomial, in agreement with Theorem 4.4

\subsection{Cluster complexes and generalized associahedra}

This section is based on [19, 21, except for the last statement in Theorem 4.11, which was proved in $\mathbf{1 3}$.

It can be shown that in a given cluster algebra of finite type, each seed is uniquely determined by its cluster. Consequently, the combinatorics of exchanges is encoded by the cluster complex, a simplicial complex (indeed, a pseudomanifold) on the set of all cluster variables whose maximal simplices (facets) are the clusters. See Figure 4.3. By Theorem 4.10, the cluster variables - hence the vertices of the cluster complex - can be naturally labeled by the set $\Phi_{\geq-1}$ of "almost positive roots" in the associated root system $\Phi$.

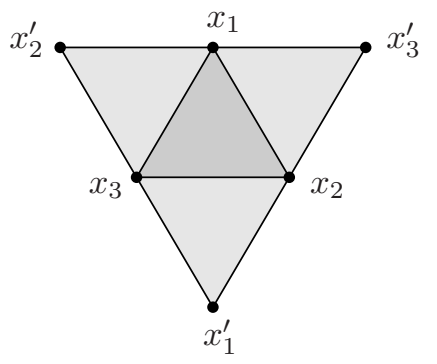

Figure 4.3. The cluster complex

This dual graph of the cluster complex is precisely the exchange graph of the cluster algebra.

Theorem 4.11 below shows that the cluster complex is always spherical, and moreover polytopal.

Recall that $Q_{\mathbb{R}}$ denotes the $\mathbb{R}$-span of $\Phi$. The $\mathbb{Z}$-span of $\Phi$ is the root lattice, denoted by $Q$.

Theorem 4.11. The $n$ roots that label the cluster variables in a given cluster form $a \mathbb{Z}$-basis of the root lattice $Q$. The cones spanned by such $n$-tuples of roots form a complete simplicial fan in the ambient real vector space $Q_{\mathbb{R}}$ (the "cluster fan"). This fan is the normal fan of a simple $n$-dimensional convex polytope in the dual space $Q_{\mathbb{R}}^{*}$.

This polytope is called the generalized associahedron of the corresponding type.

Thus, the cluster complex of a cluster algebra of finite type is canonically isomorphic to the dual simplicial complex of a generalized associahedron of the corresponding type. Conversely, the dual graph of the cluster complex is the 1-skeleton of the generalized associahedron.

\footnotetext{
${ }^{4}$ Let $P \subset V \cong \mathbb{R}^{n}$ be an $n$-dimensional simple convex polytope. The support function $F: V^{*} \rightarrow \mathbb{R}$ of $P$ is given by

$$
F(\gamma)=\max _{\mathbf{z} \in P}\langle\mathbf{z}, \gamma\rangle
$$

The normal fan $\mathcal{N}(P)$ is a complete simplicial fan in the dual space $V^{*}$ whose full-dimensional cones are the domains of linearity for $F$. More precisely, each vertex $\mathbf{z}$ of $P$ gives rise to the cone $\left\{\gamma \in V^{*}: F(\gamma)=\langle\mathbf{z}, \gamma\rangle\right\}$ in $\mathcal{N}(P)$.
} 
In type $A_{n}$, this construction recovers the $n$-dimensional associahedron (cf. Figure 4.4). The explanation involves an identification of the roots in $\Phi_{\geq-1}$ with diagonals of a convex $(n+3)$-gon that will be discussed later in Example 4.16. In type $B_{n}$, one obtains the $n$-dimensional cyclohedron. Thus the $n$-dimensional associahedron (resp., cyclohedron) is dual to the cluster complex of an arbitrary cluster algebra of type $A_{n}$ (resp., $B_{n}$ ).

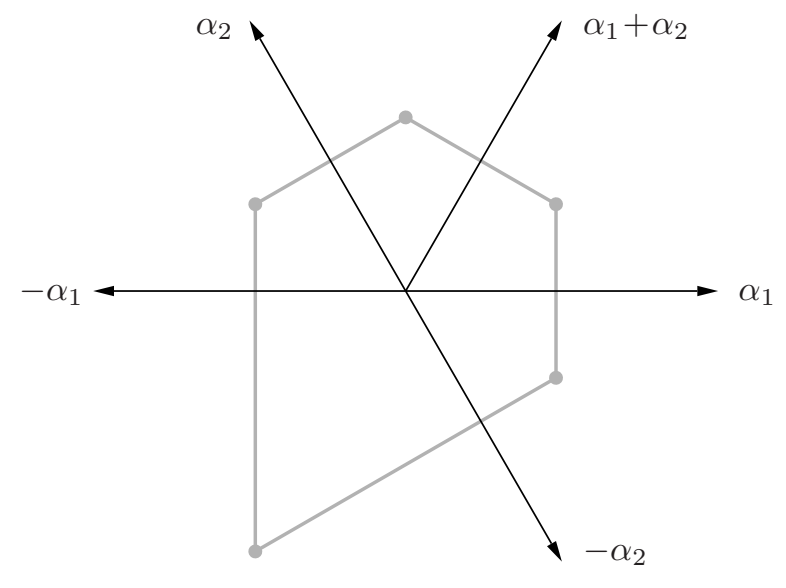

Figure 4.4. Associahedron of type $A_{2}$ and its dual fan

Theorem 4.11 leaves the following two questions unanswered:

- Which $n$-subsets of "almost positive" roots ("root clusters") label the clusters of the cluster algebra of finite type? (An answer to this question would make explicit the combinatorics of a generalized associahedron.)

- What are the inequalities defining a generalized associahedron inside $Q_{\mathbb{R}}^{*}$ ?

(We already know they are of the form $\langle\mathbf{z}, \alpha\rangle \leq$ const, for $\alpha \in \Phi_{\geq-1}$.)

We are now going to answer these questions, one after another. The answer to the first question is facilitated by the following property of a cluster complex.

Theorem 4.12. The cluster complex is a clique complex for its 1-skeleton. In other words, a subset $S \subset \Phi_{\geq-1}$ is a simplex in the cluster complex if and only if every 2-element subset of $S$ is a 1-simplex in this complex.

In type $A_{n}$, Theorem 4.12 reflects the basic property of the dual complex of an associahedron: a collection of diagonals forms a simplex if and only if any two of them do not cross.

In order to describe the cluster complex, we therefore need only to clarify which pairs of roots label the edges of the cluster complex. Thus, we need to define the root-theoretic analogue of the notion of "non-intersecting diagonals" that lies at the heart of the combinatorial construction of an associahedron.

We will assume from now on that the root system $\Phi$ underlying a cluster algebra $\mathcal{A}$ is irreducible. (The general case can be obtained by taking direct products.) We retain the notation of Lecture 2. Thus, $n$ is the rank of $\Phi$ (and $\mathcal{A}$ ); $I$ is the $n$-element indexing set, which is partitioned into disconnected pieces $I_{+}$and $I_{-}$; 
$W$ is the corresponding reflection group, generated as a Coxeter group by the generators $s_{i}$, for $i \in I ; w_{\circ}$ is the element of maximal length in $W ; A=\left(a_{i j}\right)$ is the Cartan matrix; $h$ is the Coxeter number.

Definition 4.13. Define involutions $\tau_{ \pm}: \Phi_{\geq-1} \rightarrow \Phi_{\geq-1}$ by

$$
\tau_{\varepsilon}(\alpha)= \begin{cases}\alpha & \text { if } \alpha=-\alpha_{i}, \text { for } i \in I_{-\varepsilon} \\ \prod_{i \in I_{\varepsilon}} s_{i}(\alpha) & \text { otherwise. }\end{cases}
$$

For example, in type $A_{2}$, we get:

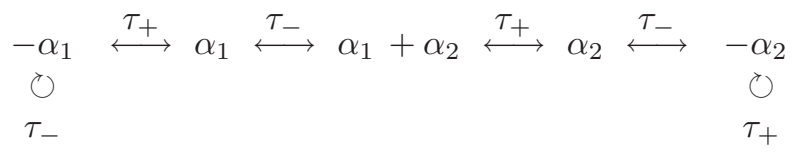

The product $\tau_{-} \tau_{+}$can be viewed as a deformation of the Coxeter element. Hence, what is the counterpart of the Coxeter number?

Theorem 4.14. The order of $\tau_{-} \tau_{+}$is $(h+2) / 2$ if $w_{\circ}=-1$, and is $h+2$ otherwise. Every $\left\langle\tau_{-}, \tau_{+}\right\rangle$-orbit in $\Phi_{\geq-1}$ has a nonempty intersection with $-\Pi$. These intersections are precisely the $\left\langle-w_{\circ}\right\rangle$-orbits in $(-\Pi)$.

Theorem 4.15. There is a unique binary relation (called "compatibility") on $\Phi_{\geq-1}$ that has the following two properties:

- $\left\langle\tau_{-}, \tau_{+}\right\rangle$-invariance: $\alpha$ and $\beta$ are compatible if and only if $\tau_{\varepsilon} \alpha$ and $\tau_{\varepsilon} \beta$ are, for $\varepsilon \in\{+,-\}$;

- a negative simple root $-\alpha_{i}$ is compatible with a root $\beta$ if and only if the simple root expansion of $\beta$ does not involve $\alpha_{i}$.

This compatibility relation is symmetric. The clique complex for the compatibility relation is canonically isomorphic to the cluster complex.

In other words (cf. Theorem 4.12), a subset of roots in $\Phi_{\geq-1}$ forms a simplex in the cluster complex if and only if every pair of roots in this subset is compatible.

Example 4.16. In type $A_{n}$, the compatibility relation can be described in concrete combinatorial terms using a particular identification of the roots in $\Phi_{\geq-1}$ with the diagonals of a regular $(n+3)$-gon. Under this identification, the roots in $-\Pi$ correspond to the diagonals on the "snake" shown in Figure 4.5. Each positive root $\alpha_{i}+\alpha_{i+1}+\cdots+\alpha_{j}$ corresponds to the unique diagonal that crosses precisely the diagonals $-\alpha_{i},-\alpha_{i+1}, \ldots,-\alpha_{j}$ from the snake (see Figure 4.6). It is easy to check that the transformations $\tau_{+}$and $\tau_{-}$act on the set of diagonals as if they were the reflections generating the dihedral group of symmetries of the $(n+3)$-gon. It then follows that two roots are compatible if and only if the corresponding diagonals do not cross each other (at an interior point).

\subsection{Polytopal realizations of generalized associahedra}

We now demonstrate how to explicitly describe each generalized associahedron by a set of linear inequalities. 


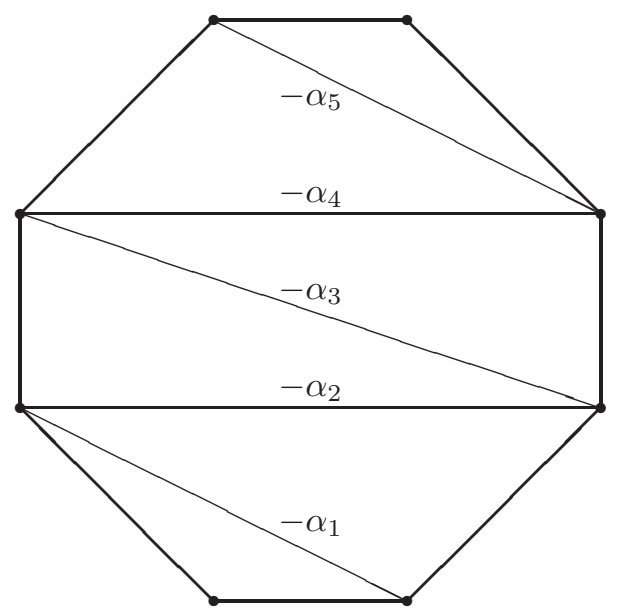

Figure 4.5. The "snake" in type $A_{5}$

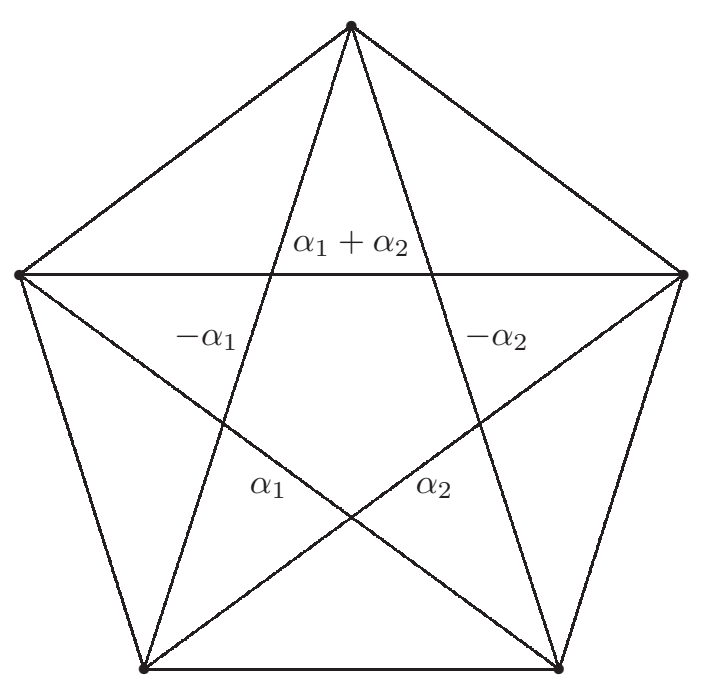

Figure 4.6. Labeling of the diagonals in type $A_{2}$

Theorem 4.17. Suppose that a $\left(-w_{\circ}\right)$-invariant function $F:-\Pi \rightarrow \mathbb{R}$ satisfies the inequalities

$$
\sum_{i \in I} a_{i j} F\left(-\alpha_{i}\right)>0 \quad \text { for all } j \in I .
$$

Let us extend $F$ (uniquely) to a $\left\langle\tau_{-}, \tau_{+}\right\rangle$-invariant function on $\Phi_{\geq-1}$. The generalized associahedron is then given in the dual space $Q_{\mathbb{R}}^{*}$ by the linear inequalities

$$
\langle\mathbf{z}, \alpha\rangle \leq F(\alpha) \text {, for all } \alpha \in \Phi_{\geq-1} .
$$

An example of a function $F$ satisfying the conditions in Theorem 4.17 is obtained by setting $F\left(-\alpha_{i}\right)$ equal to the coefficient of the simple coroot $\alpha_{i}^{\vee}$ in the 
half-sum of all positive coroots. (Coroots are the roots of the "dual" root system; see 9 , 34.)

Example 4.18. In type $A_{3}$, Theorem 4.17 is illustrated in Figure 4.7 which shows a 3 -dimensional associahedron given by the inequalities

$$
\begin{aligned}
\max \left(-z_{1},-z_{3}, z_{1}, z_{3}, z_{1}+z_{2}, z_{2}+z_{3}\right) & \leq 3 / 2, \\
\max \left(-z_{2}, z_{2}, z_{1}+z_{2}+z_{3}\right) & \leq 2 .
\end{aligned}
$$

Example 4.19. In type $C_{3}$, Theorem 4.17 is illustrated in Figure 4.8 that shows a 3 -dimensional cyclohedron given by the inequalities

$$
\begin{aligned}
\max \left(-z_{1}, z_{1}, z_{1}+z_{2}, z_{2}+z_{3}\right) & \leq 5 / 2, \\
\max \left(-z_{2}, z_{2}, z_{1}+z_{2}+z_{3}, z_{1}+2 z_{2}+z_{3}\right) & \leq 4, \\
\max \left(-z_{3}, z_{3}, 2 z_{2}+z_{3}, 2 z_{1}+2 z_{2}+z_{3}\right) & \leq 9 / 2 .
\end{aligned}
$$

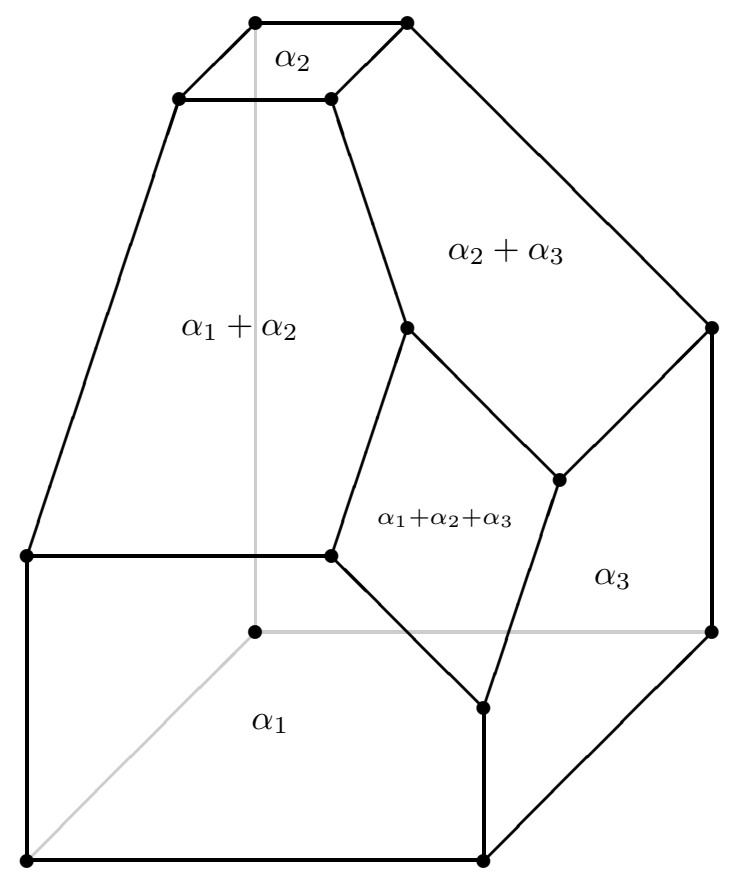

Figure 4.7. Polytopal realization of the type $A_{3}$ associahedron 


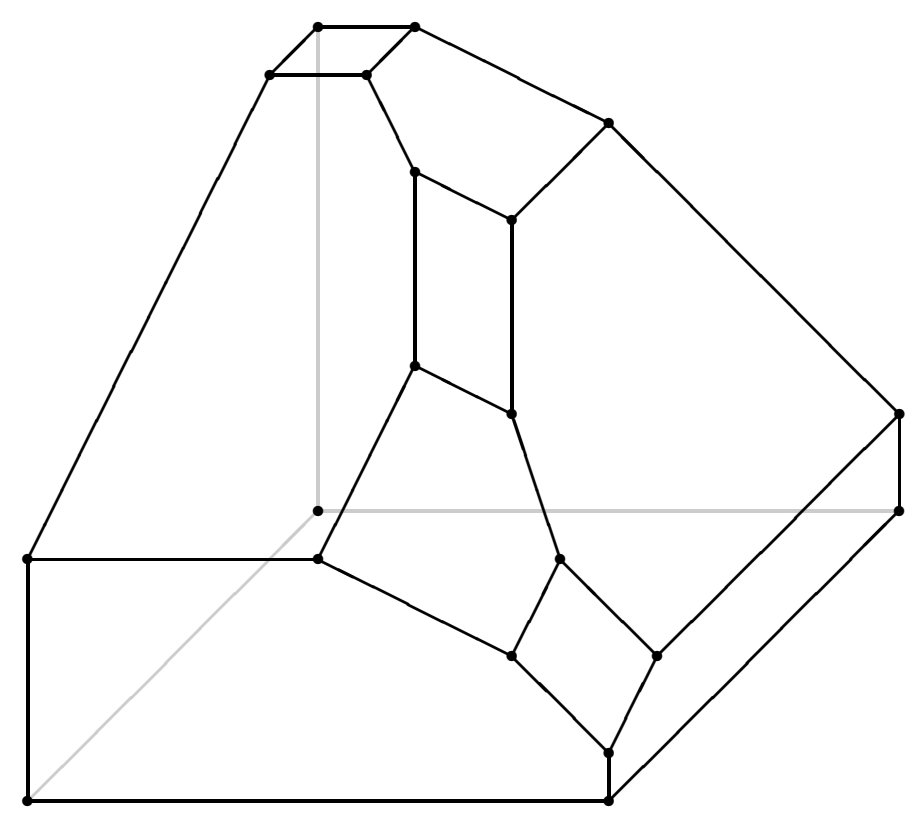

Figure 4.8. Polytopal realization of the type $C_{3}$ associahedron (cyclohedron)

\subsection{Double wiring diagrams and double Bruhat cells}

The goal of this section is to give a glimpse into how cluster algebras come up in "real life." We will present just one example: the coordinate ring of the open double Bruhat cell in $G L_{n}(\mathbb{C})$.

We will need the notion of a double wiring diagram (of type $\left(w_{\circ}, w_{\circ}\right)$ ), which is illustrated in Figure 4.9. Such a diagram consists of two families of $n$ piecewisestraight lines, each family colored with one of two colors. The crucial requirement is that each pair of lines of like color intersect exactly once. The lines in a double wiring diagram are numbered separately within each color, as shown in Figure 4.9.

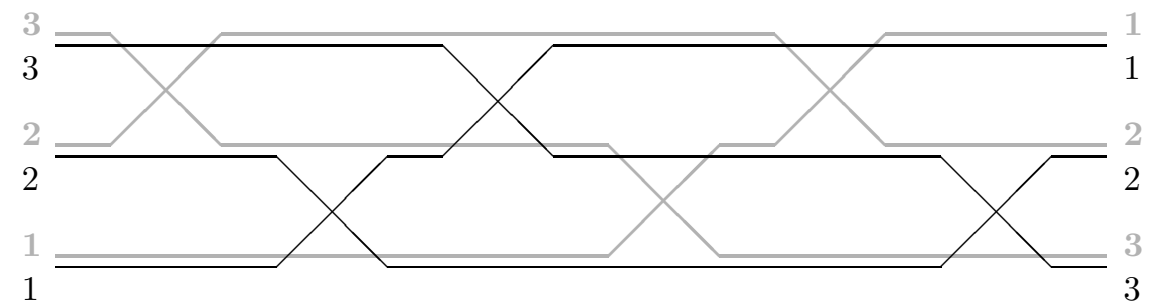

Figure 4.9. Double wiring diagram

We note in passing that double wiring diagrams correspond naturally to shuffles of two reduced words for the element $w_{\circ}$ in the symmetric group $\mathcal{S}_{n}$.

From now on, we will not distinguish between double wiring diagrams that are isotopic, i.e., have the same "topology." For example, the diagrams in Figures 4.9 and 4.10 are isotopic to each other. The diagram in Figure 4.10 is obtained from 
Figure 4.9 by sliding the two leftmost crossings past each other, and also doing the same for the two rightmost crossings.

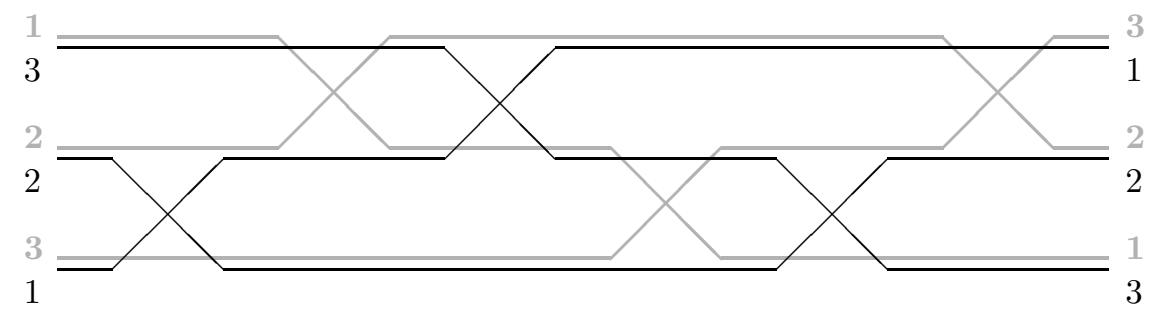

Figure 4.10. An isotopic double wiring diagram

The following lemma is a direct corollary of a theorem of G. Ringel (1956). It can also be obtained from the type $A$ version of a classical result by J. Tits (1969) concerning the word problem in Coxeter groups.

Lemma 4.20. Any two (isotopy classes of) double wiring diagrams can be transformed into each other by a sequence of local "moves" of three different kinds, shown in Figure 4.11. (Each of these local moves only changes a small portion of a double wiring diagram, leaving the rest of it intact.)

The reader is asked to ignore, for now, the labels $A, B, \ldots, Z$ in Figure 4.11

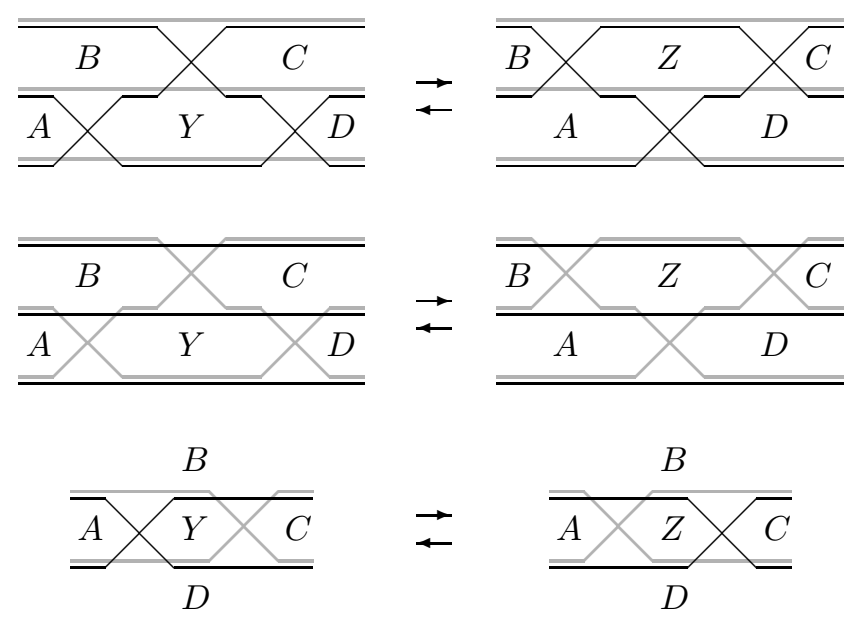

Figure 4.11. Local "moves"

To illustrate Lemma 4.20, the double wiring diagram in Figure4.9 allows 4 different local moves, all of which are of the kind shown at the bottom of Figure 4.11. Two of these moves can be performed by first passing to the isotopic Figure 4.10 To make each of the other two moves, slide the two innermost crossings in Figure 4.9 past each other; this will create two patterns of the form shown at the bottom of Figure 4.11

A chamber of a double wiring diagram is a connected component of the complement to the union of the lines, with the exception of the "crumbs" made of narrow horizontal isthmuses and small triangular regions; the large component at 
the very bottom is not included either. With these conventions, there are exactly $n^{2}$ chambers altogether (e.g., 9 chambers in Figure 4.9). We then assign to every chamber a pair of subsets of the set $[1, n]=\{1, \ldots, n\}$ : each subset indicates which lines of the corresponding color pass below that chamber; see Figure 4.12

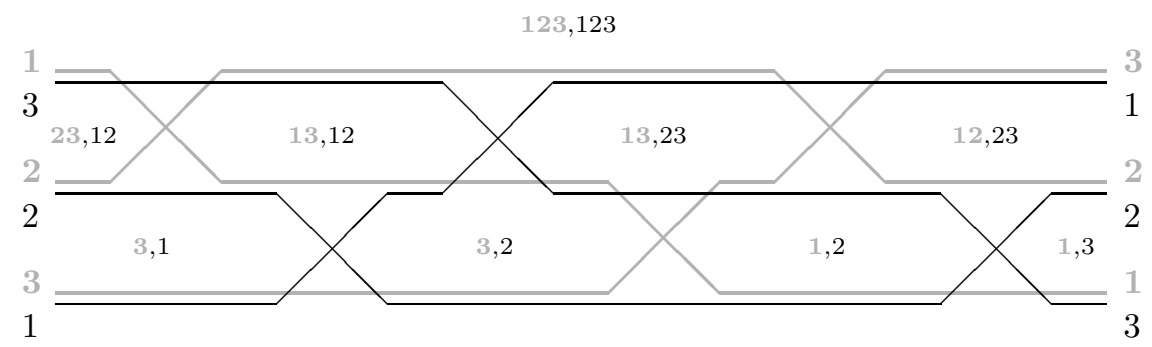

Figure 4.12. Chamber minors

Suppose we are given an $n \times n$ matrix $x=\left(x_{i j}\right)$. For any subsets $I, J \subset$ $\{1, \ldots, n\}$ of equal cardinality, we denote by $\Delta_{I, J}(x)$ the corresponding minor of $x$, that is, the determinant of the submatrix of $x$ occupying the rows and columns specified by the sets $I$ and $J$. Then each chamber of a double wiring diagram is naturally associated with a chamber minor $\Delta_{I, J}$ (viewed as a function on the general linear group $G L_{n}(\mathbb{C})$ ), where $I$ and $J$ are the sets written into that chamber.

We note that two double wiring diagrams have the same associated collections of chamber minors if and only if they are isotopic.

Let $\mathcal{F}$ denote the field of rational functions on $G L_{n}(\mathbb{C})$, i.e., the field of rational functions with complex coefficients in the matrix entries $x_{i j}$ (viewed as indeterminates).

Lemma 4.21. The $n^{2}$ chamber minors of an arbitrary double wiring diagram form a set of algebraically independent generators of the field $\mathcal{F}$.

Notice that each local move in Figure 4.11 exchanges a single chamber minor $Y$ (associated with a bounded, or interior, chamber) with another chamber minor $Z$, and keeps all other chamber minors in place. We can therefore define, by analogy with triangulations, a graph of exchanges whose vertices correspond to (isotopy classes of) double wiring diagrams, and whose edges correspond to the moves in Figure 4.11

Example 4.22. For $n=3$, there are 34 non-isotopic double wiring diagrams. The corresponding 34-vertex graph of exchanges can be found in [23. Figure 10]. It has 18 vertices of degree 4 , and 16 vertices of degree 3 . They correspond, respectively, to the double wiring diagrams that allow 4 local moves (as the diagram in Figure 4.12) and those allowing only 3 local moves (as the diagram in Figure 4.13).

Lemma 4.23. Whenever two double wiring diagrams differ by a single local move of one of the three types shown in Figure 4.11, the chamber minors appearing there satisfy the identity $A C+B D=Y Z$.

Lemmas 4.21 and 4.23 suggest the existence of a cluster algebra structure associated with $n \times n$ matrices. We next present one of several versions of this structure, leaving out most of the technical details. The ambient field for our cluster algebra is the field $\mathcal{F}$ of rational functions on $G L_{n}(\mathbb{C})$ introduced above. Each double 


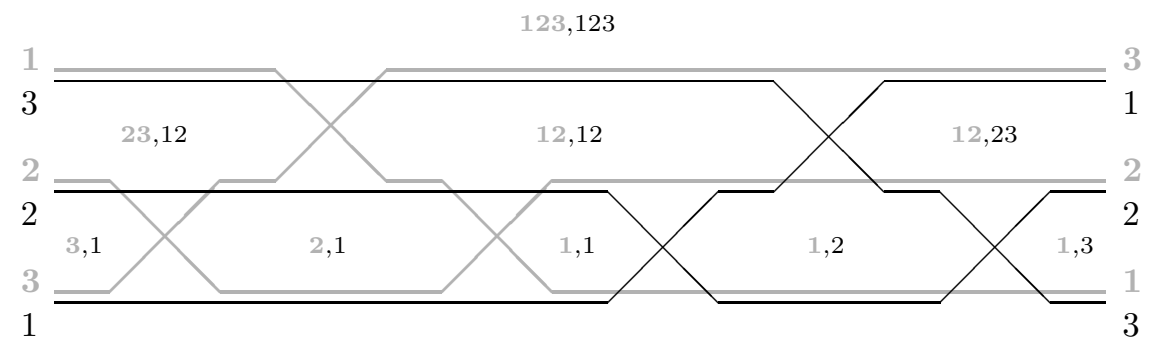

Figure 4.13. A double wiring diagram allowing 3 local moves

wiring diagram provides us with a seed whose cluster variables are the $(n-1)^{2}$ chamber minors associated with the bounded chambers; the frozen variables are the $2 n-1$ chamber minors associated with the unbounded chambers at the edges of the diagram. It remains to define the matrices $\tilde{B}$.

Take any double wiring diagram in which every bounded chamber can be "flipped" (such a diagram can be constructed for any $n$ ). Comparing the corresponding exchange relations $A C+B D=Y Z$ with (9), determine the matrix entries of $\tilde{B}$. It can be shown that exchanges associated with the local moves on double wiring diagrams are compatible with the cluster algebra axioms. Furthermore, applying these axioms uncovers hitherto hidden clusters which do not correspond to any wiring diagrams. Each variable in these clusters is a regular function on $G L_{n}(\mathbb{C})$ (a polynomial in the matrix entries). The resulting cluster algebra coincides with the coordinate ring of the open double Bruhat cell $G^{w_{\circ}, w_{\circ}}$ in $G L_{n}(\mathbb{C})$. We refer to 4 for further details.

Example 4.24. The open double Bruhat cell $G^{w_{\circ}, w_{\circ}} \subset G L_{3}(\mathbb{C})$ consists of all complex $3 \times 3$ matrices $x=\left(x_{i j}\right)$ whose minors

$$
x_{13}, \quad\left\|\begin{array}{ll}
x_{12} & x_{13} \\
x_{22} & x_{23}
\end{array}\right\|, x_{31}, \quad\left\|\begin{array}{ll}
x_{21} & x_{22} \\
x_{31} & x_{32}
\end{array}\right\|, \quad \operatorname{det}(x)
$$

are nonzero. (These 5 minors correspond to the unbounded chambers of any double wiring diagram for $G L_{3}(\mathbb{C})$.) The coordinate ring $\mathbb{C}\left[G^{w_{\circ}, w_{\circ}}\right]$ turns out to be a cluster algebra of type $D_{4}$ over the ground ring generated by the minors in (11) and their inverses. Thus, the ring of rational functions on $G L_{3}$ exhibits some quite unexpected symmetries of type $D_{4}$.

This cluster algebra has 16 cluster variables, corresponding to the 16 roots in $\Phi_{\geq-1}$. These variables are:

- 14 (among the 19 total) minors of $x$, namely, all except those listed in (11);

- two "hidden" variables: $x_{12} x_{21} x_{33}-x_{12} x_{23} x_{31}-x_{13} x_{21} x_{32}+x_{13} x_{22} x_{31}$ and $x_{11} x_{23} x_{32}-x_{12} x_{23} x_{31}-x_{13} x_{21} x_{32}+x_{13} x_{22} x_{31}$.

These 16 variables form 50 clusters of size 4 , one for each of the 50 vertices of the type $D_{4}$ associahedron.

For any $n \geq 4$, the construction described above produces a cluster algebra of infinite type. 


\section{LECTURE 5 Enumerative Problems}

\subsection{Catalan combinatorics of arbitrary type}

Let $\Phi$ be a finite irreducible crystallographic root system of rank $n$, and $W$ the corresponding reflection group. We retain the root-theoretic notation used in Lectures 2 and 4 In particular, $e_{1}, \ldots, e_{n}$ are the exponents of $\Phi$, and $h$ is the Coxeter number.

The number of vertices of an $n$-dimensional associahedron (or, equivalently, the number of clusters in a cluster algebra of type $\left.A_{n}\right)$ is the Catalan number $\frac{1}{n+2}\left(\begin{array}{c}2 n+2 \\ n+1\end{array}\right)$. It is natural to ask similar enumerative questions for other Cartan-Killing types.

Theorem 5.1 (19]). The number of clusters in a cluster algebra of finite type associated with a root system $\Phi$ (or, equivalently, the number of vertices of the corresponding generalized associahedron) is equal to

$$
N(\Phi) \stackrel{\text { def }}{=} \prod_{i=1}^{n} \frac{e_{i}+h+1}{e_{i}+1}
$$

Figure 5.1 shows the values of $N(\Phi)$ for all $\Phi$. Recall that the exponents of root systems are tabulated in Figure 2.8

\begin{tabular}{|c|c|c|c|c|c|c|c|}
\hline$A_{n}$ & $B_{n}, C_{n}$ & $D_{n}$ & $E_{6}$ & $E_{7}$ & $E_{8}$ & $F_{4}$ & $G_{2}$ \\
\hline$\frac{1}{n+2}\left(\begin{array}{c}2 n+2 \\
n+1\end{array}\right)$ & $\left(\begin{array}{c}2 n \\
n\end{array}\right)$ & $\frac{3 n-2}{n}\left(\begin{array}{c}2 n-2 \\
n-1\end{array}\right)$ & 833 & 4160 & 25080 & 105 & 8 \\
\hline
\end{tabular}

Figure 5.1. The numbers $N(\Phi)$

As the numbers $N(\Phi)$ given by (12) can be thought of as generalizations of the Catalan numbers to an arbitrary Cartan-Killing type, it comes as no surprise that they count a host of various combinatorial objects related to the root system $\Phi$. Below in this section, we briefly describe several families of objects counted by $N(\Phi)$. We refer the reader to the introductory sections of $[\mathbf{1}, \mathbf{3}, \mathbf{2}, \mathbf{1 2}, \mathbf{3 9}$, for the history of research in this area, for further details and references, and for numerous generalizations and connections.

The numbers $N(\Phi)$ seem to have first appeared in D. Djoković's work 18 on enumeration of conjugacy classes of elements of finite order in Lie groups. 
Antichains in the root poset (non-nesting partitions)

The root poset of $\Phi$ is the partial order on the set of positive roots $\Phi_{+}$such that $\beta \leq \gamma$ if and only if $\gamma-\beta$ is a nonnegative (integer) linear combination of simple roots. See Figures 5.2 and 5.3 .

Theorem 5.2 (11, 43, 46]). The number of antichains (i.e., sets of pairwise non-comparable elements) in the root poset of $\Phi$ is equal to $N(\Phi)$.

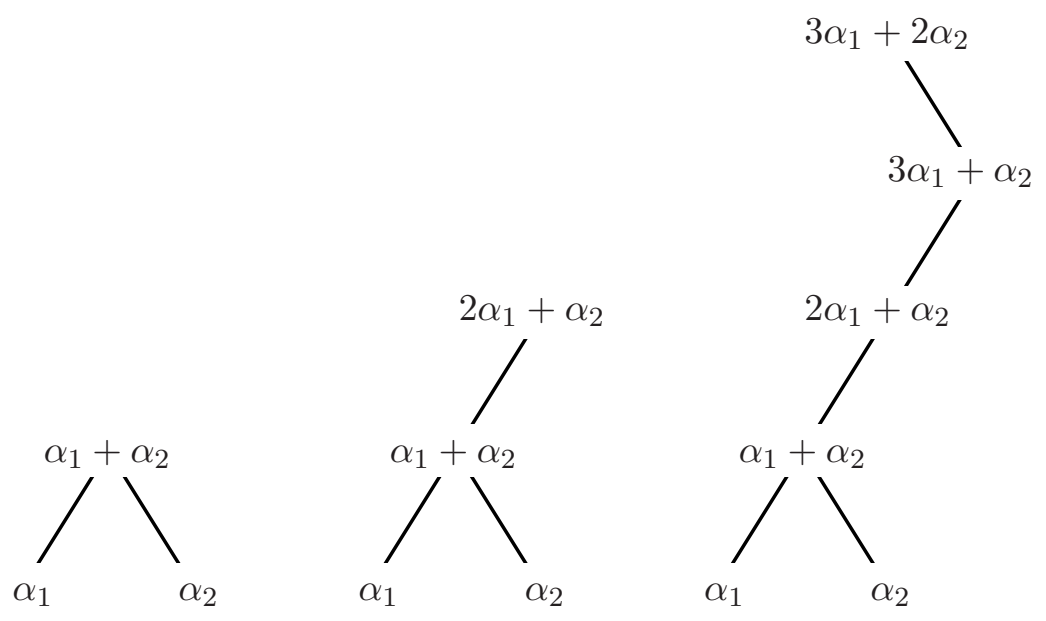

Figure 5.2. The root posets of types $A_{2}, B_{2}$ and $G_{2}$.

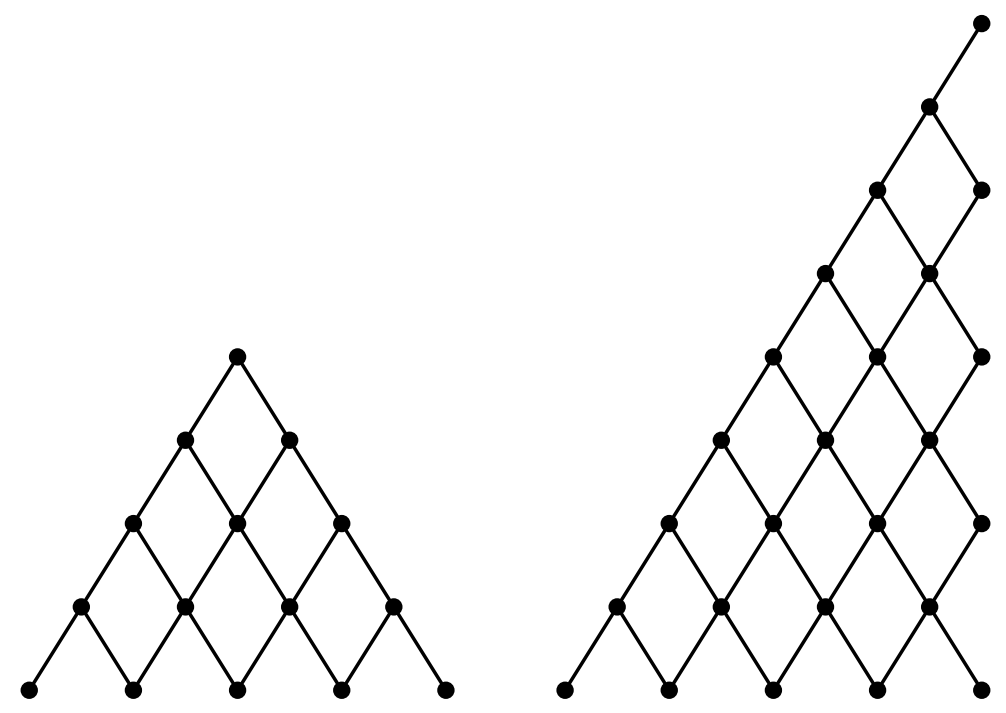

Figure 5.3. The root posets of types $A_{5}$ and $B_{5}$. 


\section{Positive regions of the Shi arrangement}

The Shi arrangement is the arrangement of affine hyperplanes defined by the equations

$$
\begin{aligned}
& \langle\beta, x\rangle=0 \quad \text { for all } \beta \in \Phi_{+} . \\
& \langle\beta, x\rangle=1
\end{aligned}
$$

(Thus, the number of hyperplanes in the Shi arrangement is equal to the number of roots in the root system $\Phi$.) The positive regions of this arrangement are the regions contained in the positive cone, which consists of the points $x$ such that $\langle\beta, x\rangle>0$ for any $\beta \in \Phi_{+}$.

Theorem 5.3 (46). The number of positive regions in the Shi arrangement is equal to $N(\Phi)$.

Figure 5.4 shows the Shi arrangements of types $A_{2}, B_{2}$ and $G_{2}$, oriented so as to agree with the root systems as drawn in Figure 1.6
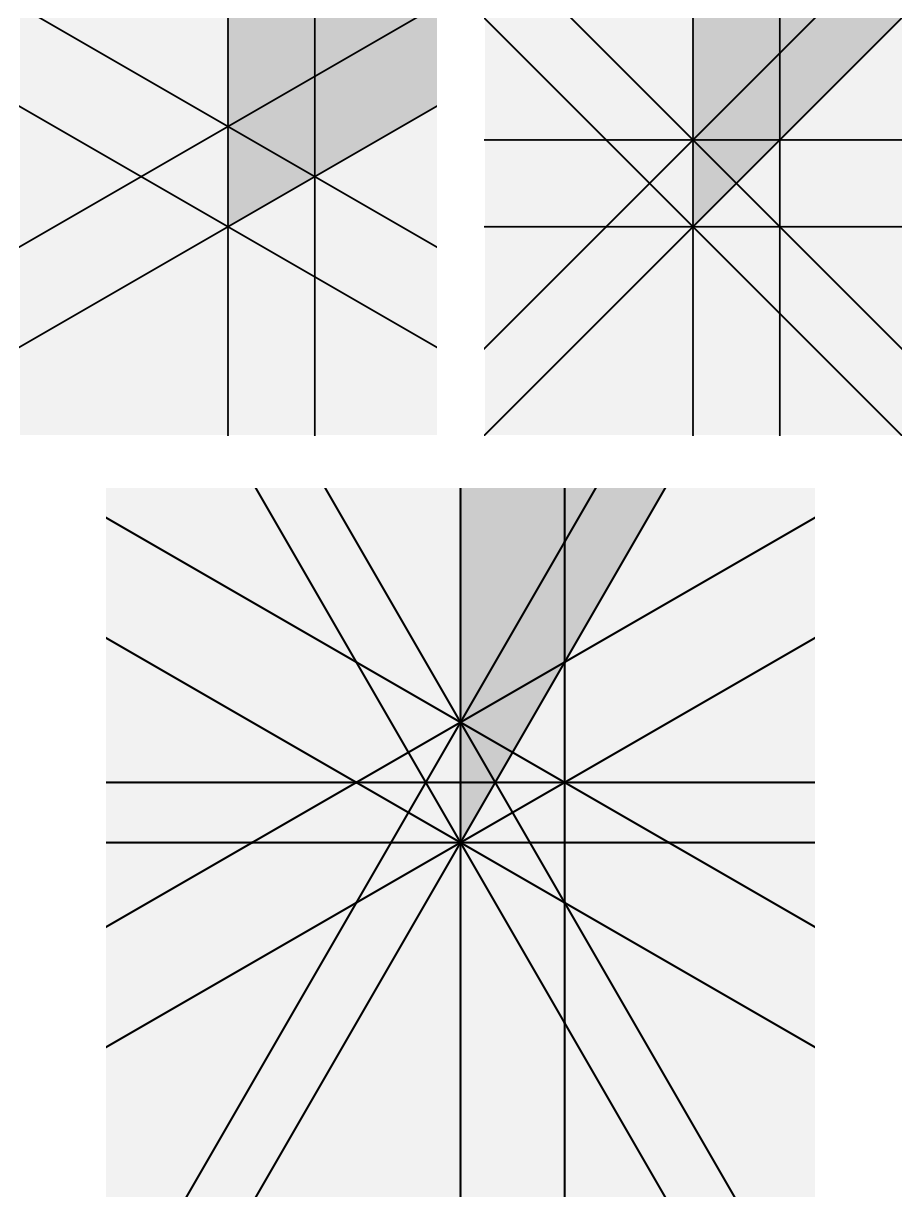

Figure 5.4. The Shi arrangements of types $A_{2}, B_{2}$ and $G_{2}$. The positive cone is shaded. 


\section{$W$-orbits in a discrete torus}

The reflection group $W$ acts on the root lattice $Q=\mathbb{Z} \Phi$, hence on the "discrete torus" $Q /(h+1) Q$ obtained as a quotient of $Q$ by its subgroup $(h+1) Q$.

Theorem $5.4([32)$. The number of $W$-orbits in $Q /(h+1) Q$ is equal to $N(\Phi)$.

Figures 5.5 and 5.6 illustrate these orbits in types $A_{2}$ and $B_{2}$, where $h=3$ and $h=4$, respectively. Each figure shows the reflection lines of the Coxeter arrangement; the shaded region is a fundamental domain for the translations in $(h+1) Q$.

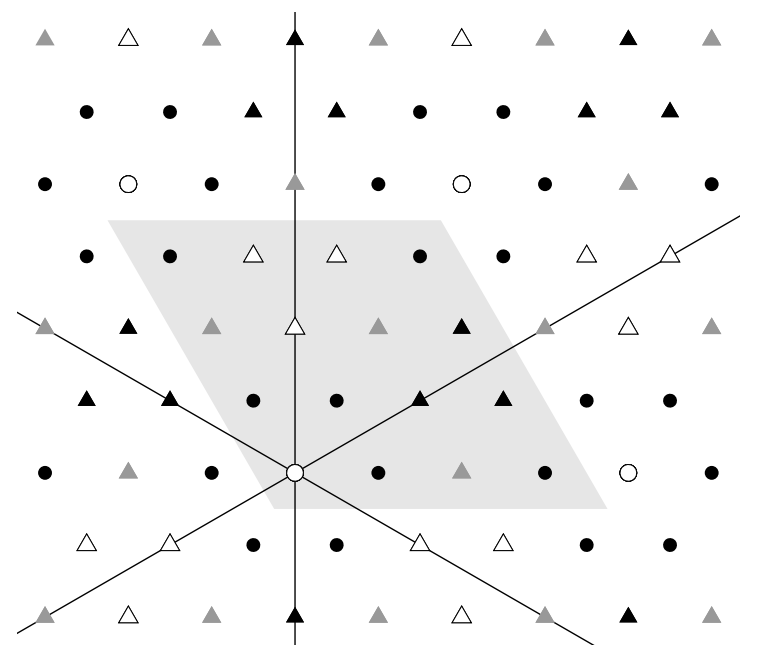

Figure 5.5. $A_{2}$-orbits in $Q / 4 Q$. Each orbit is labeled by a different symbol.

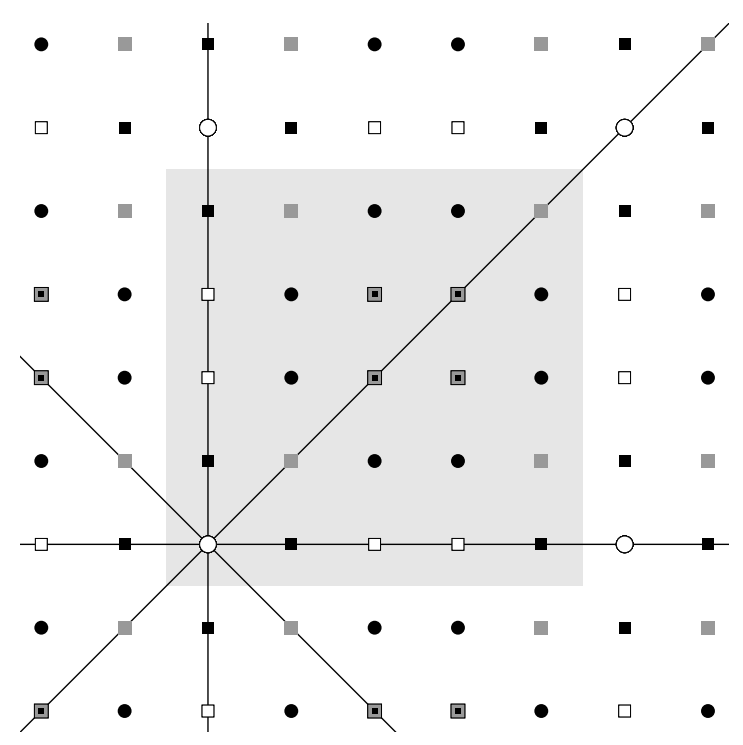

Figure 5.6. $B_{2}$-orbits in $Q / 5 Q$. 


\section{Non-crossing partitions}

The classical non-crossing partitions introduced by Kreweras are (unordered) partitions of the set $[n+1]=\{1, \ldots, n+1\}$ into non-empty subsets called blocks which satisfy the following "non-crossing" condition:

- there does not exist an ordered quadruple $(a<b<c<d)$ such that the two-element sets $\{a, c\}$ and $\{b, d\}$ are contained in different blocks.

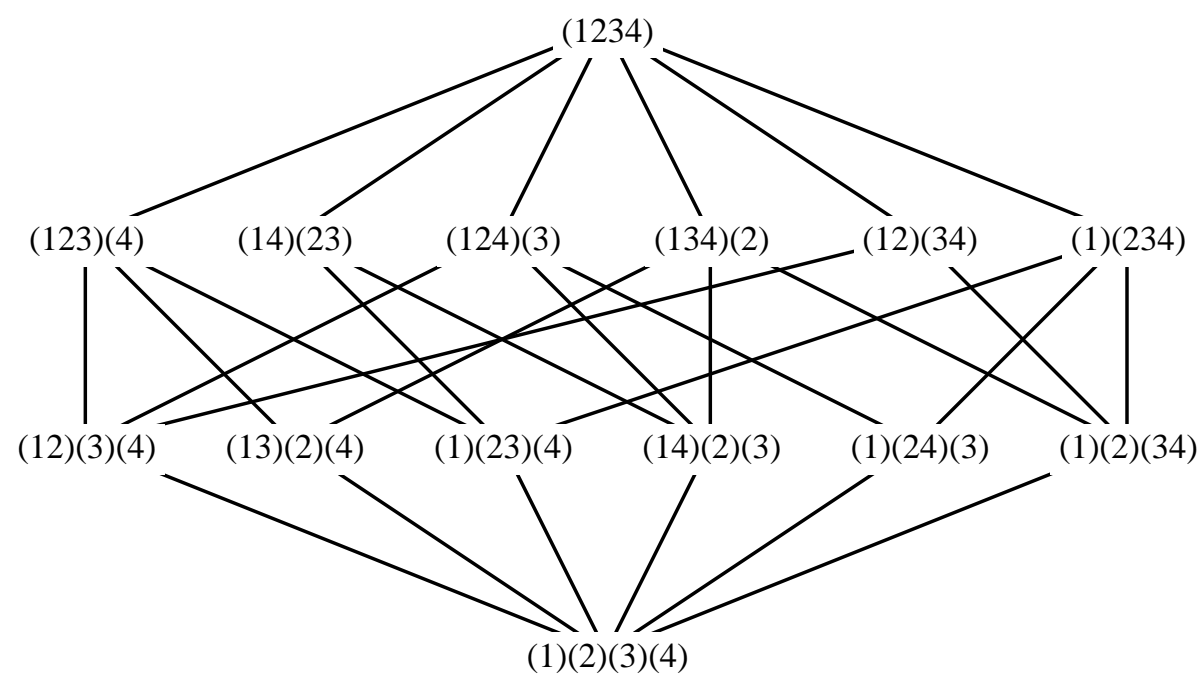

Figure 5.7. The non-crossing partition lattice of type $A_{3}$
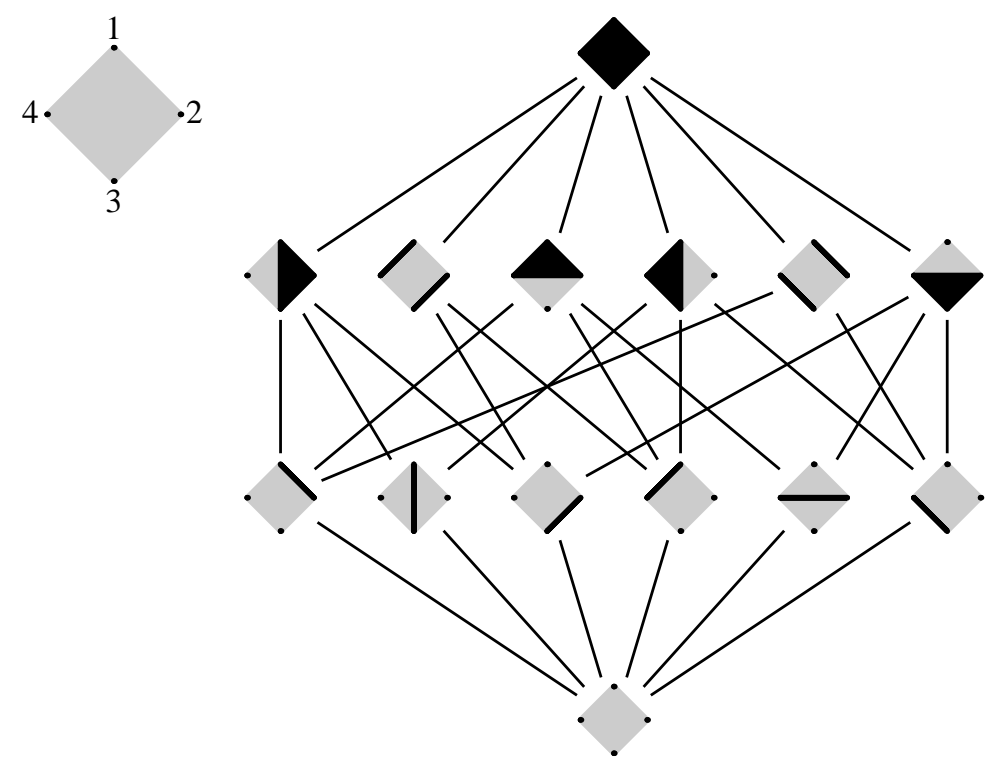

Figure 5.8. Planar representation of non-crossing partitions 
Figure 5.7 shows the 14 non-crossing partitions for $n=3$, partially ordered by refinement. Such partial order is in fact a lattice for any $n$; the number of non-crossing partitions is a Catalan number.

An alternative way of representing non-crossing partitions is shown in Figure 5.8. Place the elements of $[n+1]$ around a circle. Then the non-crossing partitions are those set partitions in which the convex hulls of blocks do not intersect.

We will now explain how this construction arises as a type- $A$ special case of a general construction valid for any (possibly infinite) Coxeter system $(W, S)$.

A reflection in a Coxeter group $W$ is an element conjugate to a generator $s \in S$. Any element $w \in W$ can be written as a product of reflections. Let $L(w)$ denote the length (i.e., number of factors) of a shortest such factorization. We then partially order $W$ by setting $u \preceq u v$ whenever $L(u v)=L(u)+L(v)$, i.e., whenever concatenating shortest factorizations for $u$ and $v$ gives a shortest factorization for $u v$. Equivalently, $w$ covers $u$ in this partial order if and only if $L(w)=L(u)+1$ and there is a reflection $t$ such that $w=u t$.

Let $c$ be a product (in an arbitrary order) of the generators in $S$. Thus, $c$ is a Coxeter element in $W$, in the broader sense of the notion alluded to in a footnote in Section 2.5. The non-crossing partition lattice for $W$ (see [7, 10) is the interval $[1, c]$ in the partial order $(W, \preceq)$ defined above. It is a classical result that all Coxeter elements are conjugate to each other. Since the set of all reflections is fixed under conjugation, it follows that different choices of $c$ yield isomorphic posets. (These posets are lattices, which is a non-trivial theorem.)

The following theorem was obtained in [7, 40. A version for the classical types $A B C D$ appeared earlier in 43 .

Theorem 5.5. Let $W$ be the reflection group associated with a finite root system $\Phi$. Then the non-crossing partition lattice for $W$ has $N(\Phi)$ elements.

In type $A_{n}$, the general construction presented above recovers the ordinary noncrossing partition lattice. To realize why, look again at Figure 5.7 and interpret each element of the poset as a permutation in $\mathcal{S}_{4}$ written in cycle notation.

The non-crossing partition lattice of type $B_{n}$ can also be given a direct combinatorial description. Let us take the ordinary lattice of non-crossing partitions of a $2 n$-element set in its representation illustrated in Figure 5.8. Then consider the sublattice consisting of those partitions whose planar representations are centrally symmetric. The result (for $n=3$ ) is shown in Figure 5.9.

\subsection{Generalized Narayana Numbers}

For any enumerative problem whose answer is a Catalan number, replacing a simple count by a generating function with respect to some combinatorial statistic results in a $q$-analogue of a Catalan number. There are at least three such $q$-analogues that routinely pop up in various contexts. One is obtained from the usual formula $\frac{1}{n+2}\left(\begin{array}{c}2 n+2 \\ n+1\end{array}\right)$ by replacing $n+2$ and $\left(\begin{array}{c}2 n+2 \\ n+1\end{array}\right)$ with their standard $q$-analogues. A different answer is obtained while counting order ideals in the root poset of type $A_{n}$ by the cardinality of an ideal. For more on these $q$-analogues, see [26, 27, [51.

We will focus on a third $q$-analogue that is related to the Narayana numbers, defined by the formula $\frac{1}{n+1}\left(\begin{array}{c}n+1 \\ k\end{array}\right)\left(\begin{array}{c}n+1 \\ k+1\end{array}\right)$. The Narayana numbers form a triangle shown on the right in Figure 5.10 . Thus, the numbers in each row of this triangle 


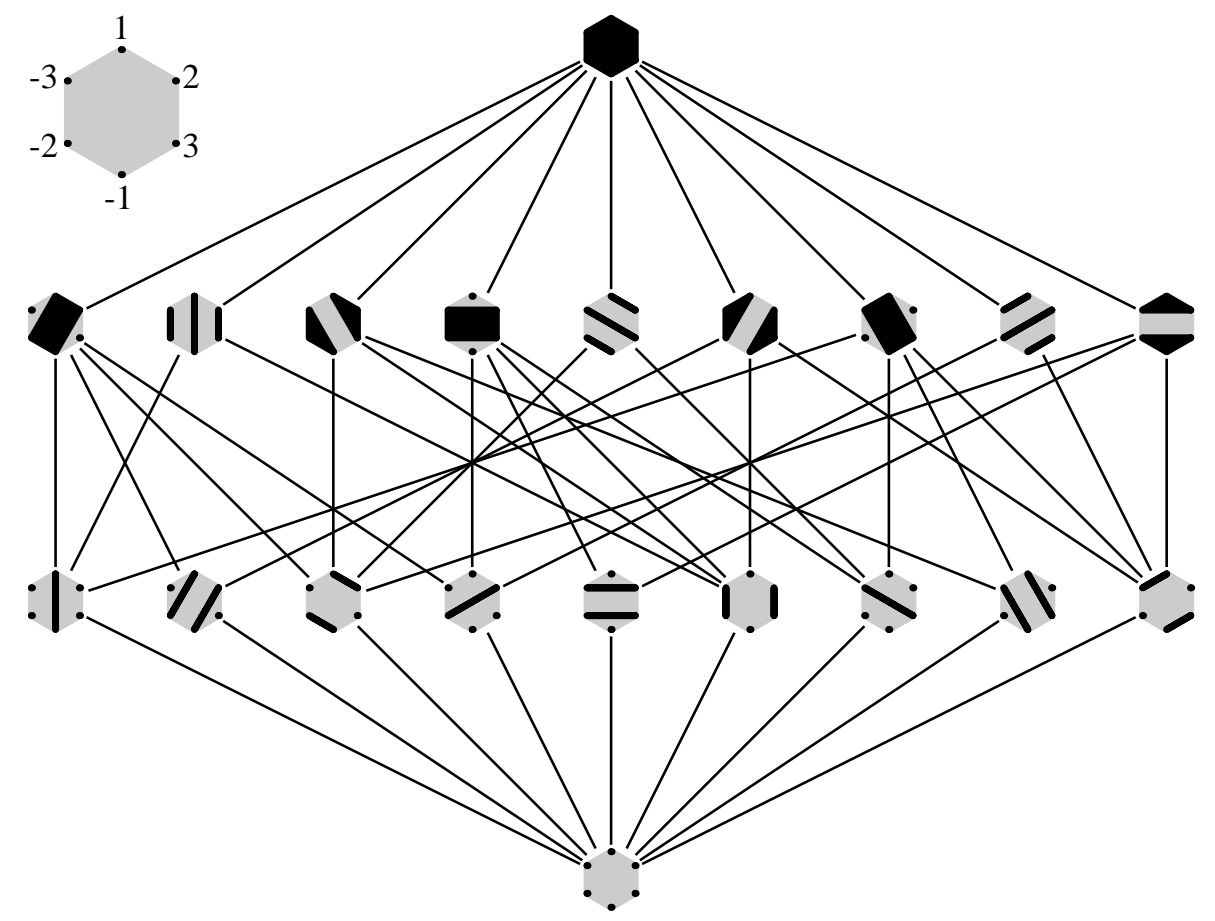

Figure 5.9. The non-crossing partitions of type $B_{3}$.

are obtained by looking at the corresponding row of Pascal's triangle on the left, computing products of consecutive pairs of entries, and dividing them by $n+1$.

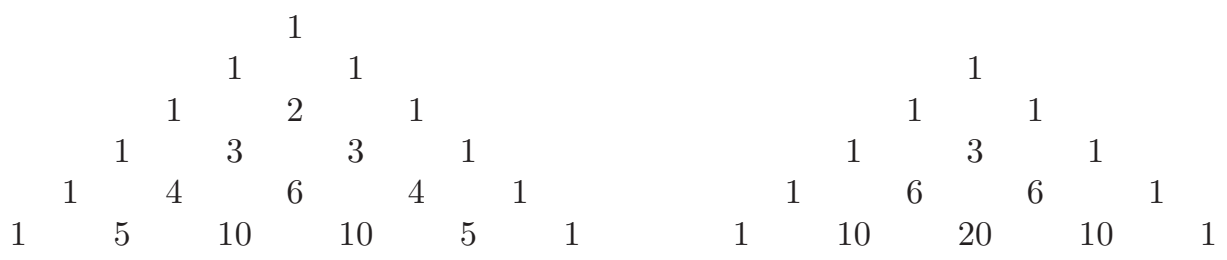

Figure 5.10. The Pascal triangle and the Narayana numbers

Remarkably, the row sums in the triangle of Narayana numbers are the Catalan numbers:

$$
\sum_{k=0}^{n} \frac{1}{n+1}\left(\begin{array}{c}
n+1 \\
k
\end{array}\right)\left(\begin{array}{l}
n+1 \\
k+1
\end{array}\right)=\frac{1}{n+2}\left(\begin{array}{c}
2 n+2 \\
n+1
\end{array}\right) .
$$

This suggests introducing a $q$-analogue of the Catalan numbers given by

$$
\sum_{k=0}^{n} \frac{1}{n+1}\left(\begin{array}{c}
n+1 \\
k
\end{array}\right)\left(\begin{array}{c}
n+1 \\
k+1
\end{array}\right) q^{k}
$$

We will now explain the connection between this $q$-analogue and the classical (type $A$ ) associahedron. This connection will lead us to an extension of the definition to other root systems. 
We will need the notions of the $f$-vector and $h$-vector of an $(n-1)$-dimensional simplicial complex. The $f$-vector is $\left(f_{-1}, f_{0}, \ldots, f_{n-1}\right)$ where $f_{i}$ denotes the number of $i$-dimensional faces. The unique "(-1)-dimensional" face is the empty face. The $h$-vector $\left(h_{0}, h_{1}, \ldots, h_{n}\right)$ is determined from the $f$-vector by the "reverse Pascal's triangle" recursion which we illustrate by an example.

Example 5.6. The $f$-vector of the simplicial complex dual to the associahedron of type $A_{3}$ is $(1,9,21,14)$. (See Figure 3.6.) To calculate the $h$-vector, we place the $f$-vector and a row of 1's in a triangular array as shown in Figure 5.11 on the left, with most of the entries as yet undetermined. The remaining entries are then filled in by applying the following rule: each entry is the difference between the entry preceding it in its row and the entry directly southwest of it. Thus, we get $9-1=8,21-8=13$, etc. Finally, we obtain the $h$-vector $(1,6,6,1)$ by reading the rightmost entries in every row. Notice that these are exactly the Narayana numbers appearing in the third row in Figure 5.10 .

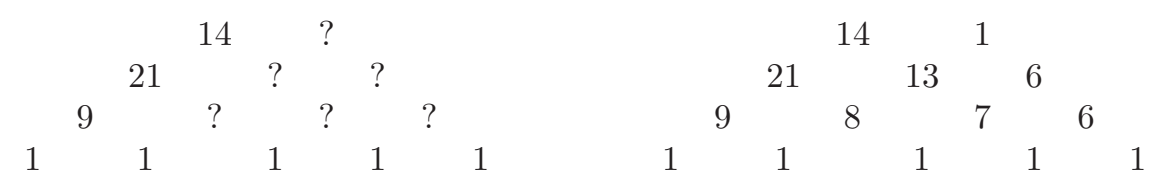

Figure 5.11. Computing the $h$-vector

Lemma 5.7. The components of the h-vector of the simplicial complex dual to an $n$-dimensional associahedron are the Narayana numbers $\frac{1}{n+1}\left(\begin{array}{c}n+1 \\ k\end{array}\right)\left(\begin{array}{c}n+1 \\ k+1\end{array}\right)$.

Motivated by Lemma 5.7, we define the (generalized) Narayana numbers $N_{k}(\Phi)$ $(k=0, \ldots, n)$ for an arbitrary root system $\Phi$ as the entries of the $h$-vector of the simplicial complex dual to the corresponding generalized associahedron.

Example 5.8. The $f$-vector of the simplicial complex dual to the 3-dimensional cyclohedron (the associahedron of type $\left.B_{3}\right)$ is $(1,12,30,20)$. The corresponding $h$-vector is $(1,9,9,1)$. In general, the Narayana numbers of type $B_{n}$ are the squares of entries of Pascal's triangle: $N_{k}\left(B_{n}\right)=\left(\begin{array}{l}n \\ k\end{array}\right)^{2}$.

It is easy to see that the entries of an $h$-vector always add up to $f_{n-1}$, the number of top-dimensional faces in the simplicial complex. Thus, $\sum_{k} N_{k}(\Phi)=N(\Phi)$. Consequently, the generating function for the Narayana numbers of type $\Phi$

$$
N(\Phi, q)=\sum_{k=0}^{n} N_{k}(\Phi) q^{k}
$$

provides a $q$-analogue of $N(\Phi)$ which generalizes (13). These generating functions for the finite crystallographic root systems are tabulated in Figure 5.12.

The Narayana numbers provide refined counts for the various interpretations of $N(\Phi)$ given in Section 5.1. These enumerative results are listed in Theorem 5.9 below; we elaborate on the items in the theorem in subsequent comments.

Theorem 5.9 is a combination of results in $[2,19,39,44,48$; see 2 for a historical overview, and for further generalizations. 


$$
\begin{aligned}
& N\left(A_{n}, q\right)=\sum_{k=0}^{n} \frac{1}{n+1}\left(\begin{array}{c}
n+1 \\
k
\end{array}\right)\left(\begin{array}{c}
n+1 \\
k+1
\end{array}\right) q^{k} \\
& N\left(B_{n}, q\right)=\sum_{k=0}^{n}\left(\begin{array}{l}
n \\
k
\end{array}\right)^{2} q^{k} \\
& N\left(D_{n}, q\right)=1+q^{n}+\sum_{k=1}^{n-1}\left[\left(\begin{array}{l}
n \\
k
\end{array}\right)^{2}-\frac{n}{n-1}\left(\begin{array}{c}
n-1 \\
k-1
\end{array}\right)\left(\begin{array}{c}
n-1 \\
k
\end{array}\right)\right] q^{k} \\
& N\left(E_{6}, q\right)=1+36 q+204 q^{2}+351 q^{3}+204 q^{4}+36 q^{5}+q^{6} \\
& N\left(E_{7}, q\right)=1+63 q+546 q^{2}+1470 q^{3}+1470 q^{4}+546 q^{5}+63 q^{6}+q^{7} \\
& N\left(E_{8}, q\right)=1+120 q+1540 q^{2}+6120 q^{3}+9518 q^{4} \\
& N\left(F_{4}, q\right)=1+24 q+55 q^{2}+24 q^{3}+q^{4} \\
& N\left(G_{2}, q\right)=1+6 q+q^{2}+1540 q^{6}+120 q^{7}+q^{8}
\end{aligned}
$$

Figure 5.12. Generating functions for generalized Narayana numbers

Theorem 5.9. The following numbers are equal to each other, and to $N_{k}(\Phi)$ :

(i) the kth component of the h-vector for the dual complex of a generalized associahedron of type $\Phi$;

(ii) the number of elements of rank $k$ in the non-crossing partition lattice for $W$;

(iii) the number of antichains of size $k$ in the root poset for $\Phi$;

(iv) the number of $W$-orbits in $Q /(h+1) Q$ consisting of elements whose stabilizer has rank $k$;

(v) the components of the h-vector for the dual cell complex of the positive part of the Shi arrangement.

Remark 5.10 (Comments on Theorem [5.9) .

(i) This was our definition of $N_{k}(\Phi)$.

(ii) The lattice of non-crossing partitions of type $\Phi$ is graded, and $N_{k}(\Phi)$ is the number of elements of rank $k$.

(iii) The $h$-vector of any simplicial polytope satisfies the Dehn-Sommerville equations $h_{i}=h_{d-i}$. Thus interpretation (i) implies that $N_{k}(\Phi)=N_{n-k}(\Phi)$. This symmetry of the Narayana numbers is also apparent in the interpretation (ii) because the non-crossing partition lattices are self-dual. However, this symmetry is not at all obvious in the interpretations (iii)-(v). In particular, no direct combinatorial explanation is known for why the number of antichains of size $k$ in the root poset is the same as the number of antichains of size $n-k$.

(iv) The stabilizer of an element in $Q /(h+1) Q$ is a reflection subgroup of $W$. The stabilizers of elements in the same $W$-orbit are conjugate, and therefore have the same rank. $N_{k}(\Phi)$ is the number of orbits in which the stabilizers have rank $k$. For example, in type $A_{2}$ there is 1 orbit whose stabilizer has rank 2 (the unfilled circle in Figure 5.5), 3 orbits whose stabilizers have rank 1 (each symbolized by a triangle) and 1 orbit whose stabilizers have rank 0 (the filled circles), in agreement with $N\left(A_{2}, q\right)=1+3 q+q^{2}$. 
(v) The positive regions of the Shi arrangement can be used to define a "dual" cell complex. The vertices of this complex correspond to the positive regions of the Shi arrangement. The faces of the complex correspond to those faces of the closures of these regions that are not contained in the boundary of the positive cone. Accordingly, the maximal faces correspond to the vertices of the arrangement which lie in the interior of the positive cone. See Figure 5.13 Amazingly, this cell complex has the same $f$-vector (hence the same $h$-vector) as the corresponding associahedron. In the example of Figure 5.13, we get 5 vertices, 5 faces, and 1 two-dimensional face, matching the numbers for the pentagon (the type $A_{2}$ associahedron).

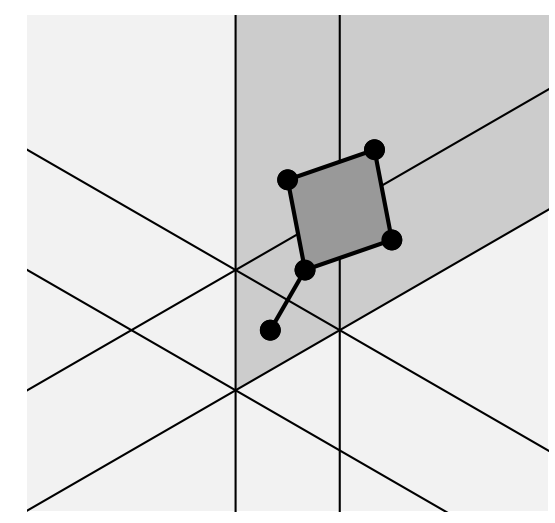

Figure 5.13. The dual complex for the positive part of the Shi arrangement of type $A_{2}$.

\subsection{Non-crystallographic types}

The construction of generalized associahedra via Definition 4.13 and Theorems 4.14 and 4.15 can be carried out verbatim for the non-crystallographic root systems $I_{2}(m), H_{3}$ and $H_{4}$. (However, the last sentence of Theorem 4.15 must be ignored, since no "cluster complex" exists for non-crystallographic root systems.) The associahedron of type $I_{2}(m)$ is an $(m+2)$-gon. The 1-skeleton of the associahedron for $\mathrm{H}_{3}$ is shown in Figure 5.14. (The vertex at infinity completes the three unbounded regions to heptagons.)

The analogue of Theorem 5.1 holds true in types $H_{3}, H_{4}$, and $I_{2}(m)$ : the number of vertices of a generalized associahedron is equal to $N(\Phi)$. The latter number is still given by (12), with the exponents taken from Figure 2.8. Figure 5.15 shows these values of $N(\Phi)$ explicitly.

The corresponding $h$-vectors ("Narayana numbers") are given by

$$
\begin{aligned}
N\left(I_{2}(m), q\right) & =1+m q+q^{2}, \\
N\left(H_{3}, q\right) & =1+15 q+15 q^{2}+q^{3}, \\
N\left(H_{4}, q\right) & =1+60 q+158 q^{2}+60 q^{3}+q^{4} .
\end{aligned}
$$

The construction of the non-crossing partition lattice does not require a crystallographic Coxeter group. Theorem 5.5] and Theorem [5.9(ii) remain valid for the finite non-crystallographic root systems. At present, the other manifestations of $N(\Phi)$ and $N_{k}(\Phi)$ presented in Sections 5.1 and 5.2 (including Parts (iii)-(v) of Theorem [5.9) do not appear to extend to the non-crystallographic cases. 


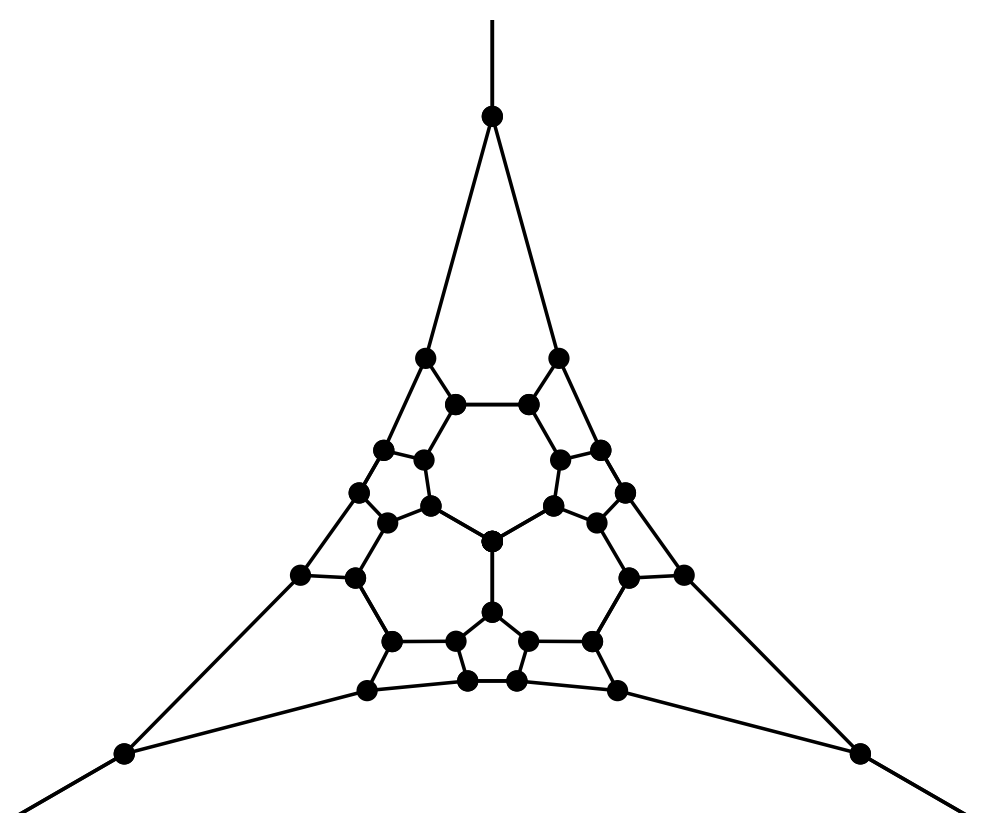

Figure 5.14. The associahedron of type $\mathrm{H}_{3}$

\begin{tabular}{|l|l|l|}
\hline$H_{3}$ & $H_{4}$ & $I_{2}(m)$ \\
\hline 32 & 280 & $m+2$ \\
\hline
\end{tabular}

Figure 5.15. The numbers $N(\Phi)$ in non-crystallographic cases

\subsection{Lattice congruences and the weak order}

This section is based on [41. Its main goal is to establish a relationship between two fans associated with a root system $\Phi$ and the corresponding reflection group $W$ :

- the Coxeter fan created by (the regions of) the Coxeter arrangement, and

- the cluster fan described in Theorem 4.11

These fans are the normal fans of a permutahedron and an associahedron of the corresponding type, respectively.

Let $\omega_{i}$ denote the fundamental weight $\left[\mathbf{9}\right.$ ] corresponding to $\alpha_{i}$. For $i \in I$, we set

$$
\varepsilon(i)= \begin{cases}+1 & \text { if } i \in I_{+} \\ -1 & \text { if } i \in I_{-}\end{cases}
$$

Theorem 5.11. The linear automorphism $Q_{\mathbb{R}} \rightarrow Q_{\mathbb{R}}$ defined by $\alpha_{i} \mapsto \varepsilon(i) \omega_{i}$ moves the cluster fan to a fan refined by the Coxeter fan.

The gluing of maximal cones of the Coxeter fan corresponds to contraction of edges in the 1-skeleton of a permutahedron. By Theorem 5.11, this can be done in such a way that the result of the contraction is the 1-skeleton of a generalized associahedron. We have thus extended Theorem 3.6 to all types. 
The statement of Theorem 5.11 does not specify which regions of the Coxeter arrangement should be combined together to produce the maximal cones of the transformed cluster fan. We next present a lattice-theoretic construction that, conjecturally, answers this question.

The weak order on $W$ is the partial order in which $u \leq v$ if and only if some reduced word for $u$ occurs as an initial segment of a reduced word for $v$. In particular, $v$ covers $u$ in the weak order if and only if $u^{-1} v$ is a simple reflection, and the length of $v$ is greater than the length of $u$ (necessarily by 1 ). Lemma 2.13 (see also the paragraph that follows it) implies that the Hasse diagram of the weak order can be identified with the 1-skeleton of a $W$-permutohedron.

Theorem $5.12(\underline{6})$. The weak order on a finite Coxeter group is a lattice.

Example 5.13. The weak order of type $A_{n}$ can be described in the language of permutations of $[n+1]$, written in one-line notation. Permutation $v=\left(v_{1}, \ldots, v_{n+1}\right)$ covers $u=\left(u_{1}, \ldots, u_{n+1}\right)$ if $v$ is obtained from $u$ by exchanging two entries $u_{i}$ and $u_{i+1}$ with $u_{i}<u_{i+1}$. Figure 5.16 shows the weak order on $A_{3}$. (Cf. Figure 2.3.)

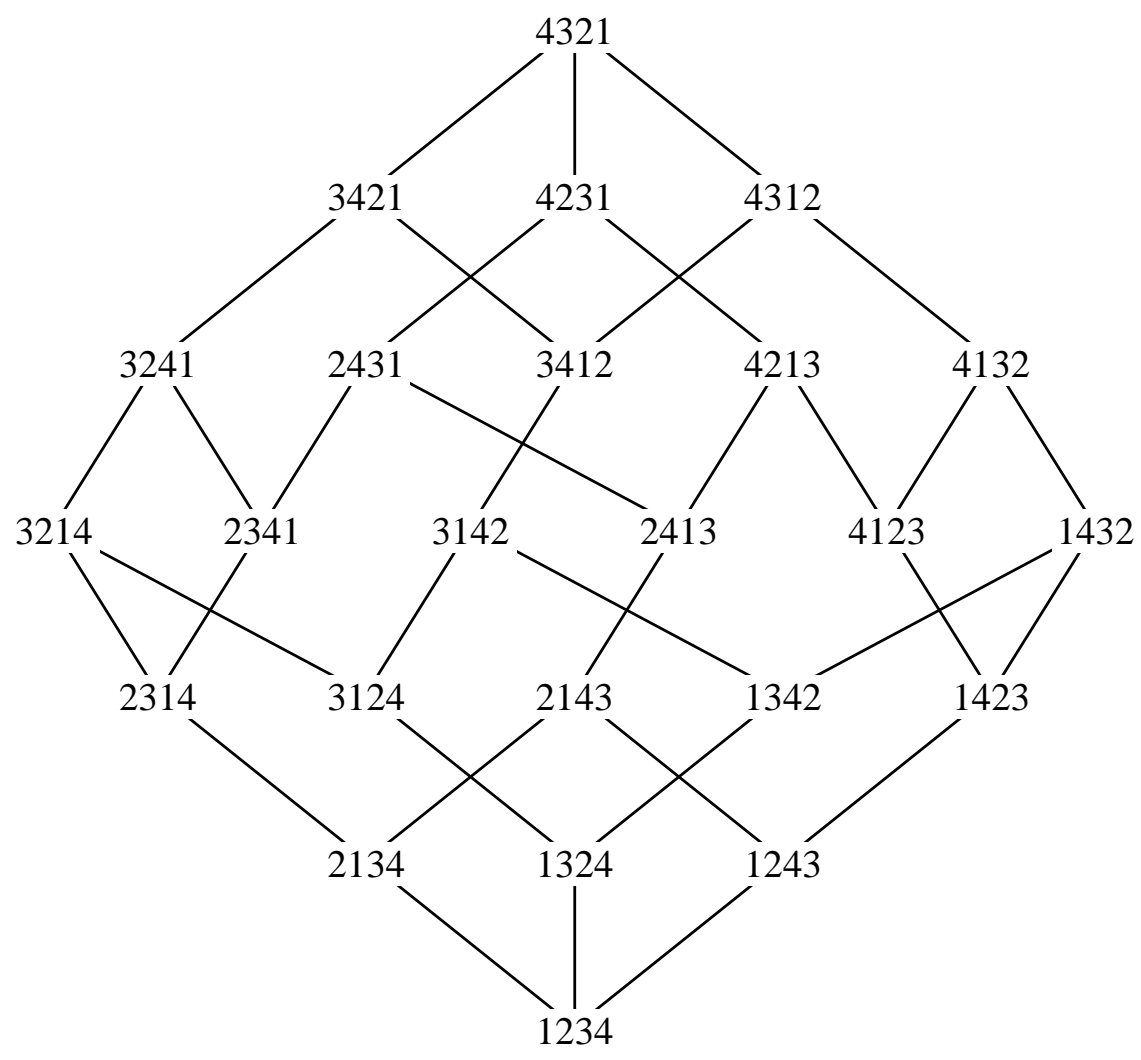

Figure 5.16. The weak order of type $A_{3}$

A congruence on a lattice is an equivalence relation which respects the meet and join operations. A (bipartite) Cambrian congruence on the weak order of $W$ is defined as the (unique) coarsest congruence "三" such that, for each edge $(s, t)$ in 
the Coxeter diagram, with $t \in I_{-}$, we have

$$
t \equiv \text { tsts } \cdots \quad\left(m_{s t}-1 \text { factors }\right) .
$$

Example 5.14. Figure 5.17 shows the bipartite Cambrian congruence for $W$ of type $A_{3}$, i.e., the coarsest congruence on the weak order of the symmetric group $\mathcal{S}_{4}$ such that $1324 \equiv 3124$ and $1324 \equiv 1342$. The congruence classes are shaded.

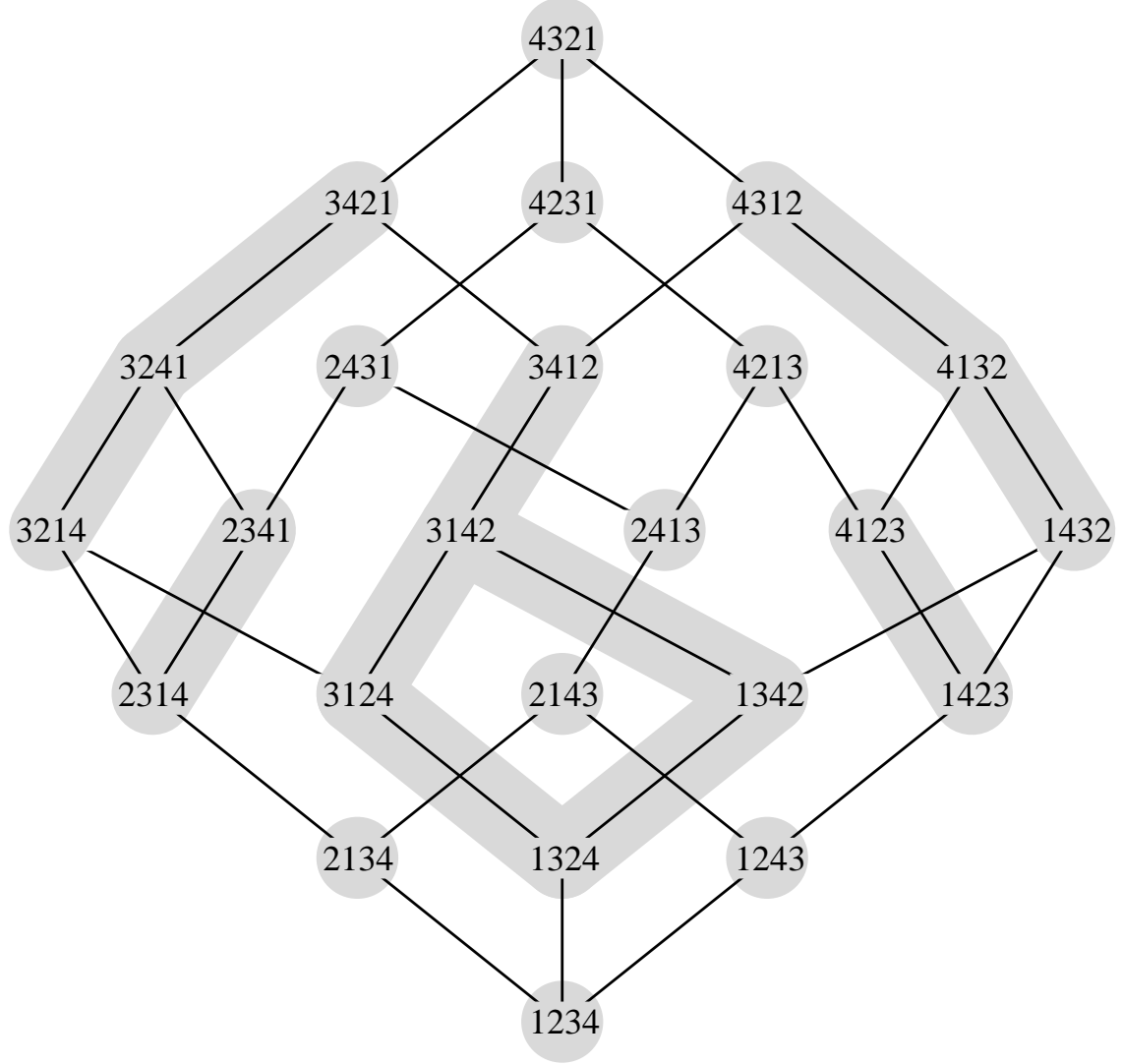

Figure 5.17. A bipartite Cambrian congruence of type $A_{3}$

Conjecture 5.15. Two regions $R_{u}$ and $R_{v}$ of the Coxeter arrangement are contained in the same maximal cone of the transformed cluster fan (see Theorem 5.11) if and only if $u \equiv v$ under the bipartite Cambrian congruence.

Conjecture 5.15 has been proved in types $A_{n}$ and $B_{n}$. The proof makes explicit the combinatorics of the Cambrian congruence and connects it to constructions given by Billera and Sturmfels [5] (type $A$ ) and Reiner $\mathbf{4 2}$ (type $B$ ). The conjecture implies in particular that the Hasse diagram of the quotient of the weak order by the Cambrian congruence (called the Cambrian lattice) is isomorphic to the 1-skeleton of the generalized associahedron.

More concretely, the Cambrian lattice is obtained as the induced subposet of the weak order formed by taking the (unique) smallest element in each (Cambrian) congruence class; see Figure 5.18. We omit the description of the bijection used to 
translate the top picture in Figure 5.18 (the Cambrian lattice labeled by permutations) into the bottom one (the associahedron labeled by triangulations).
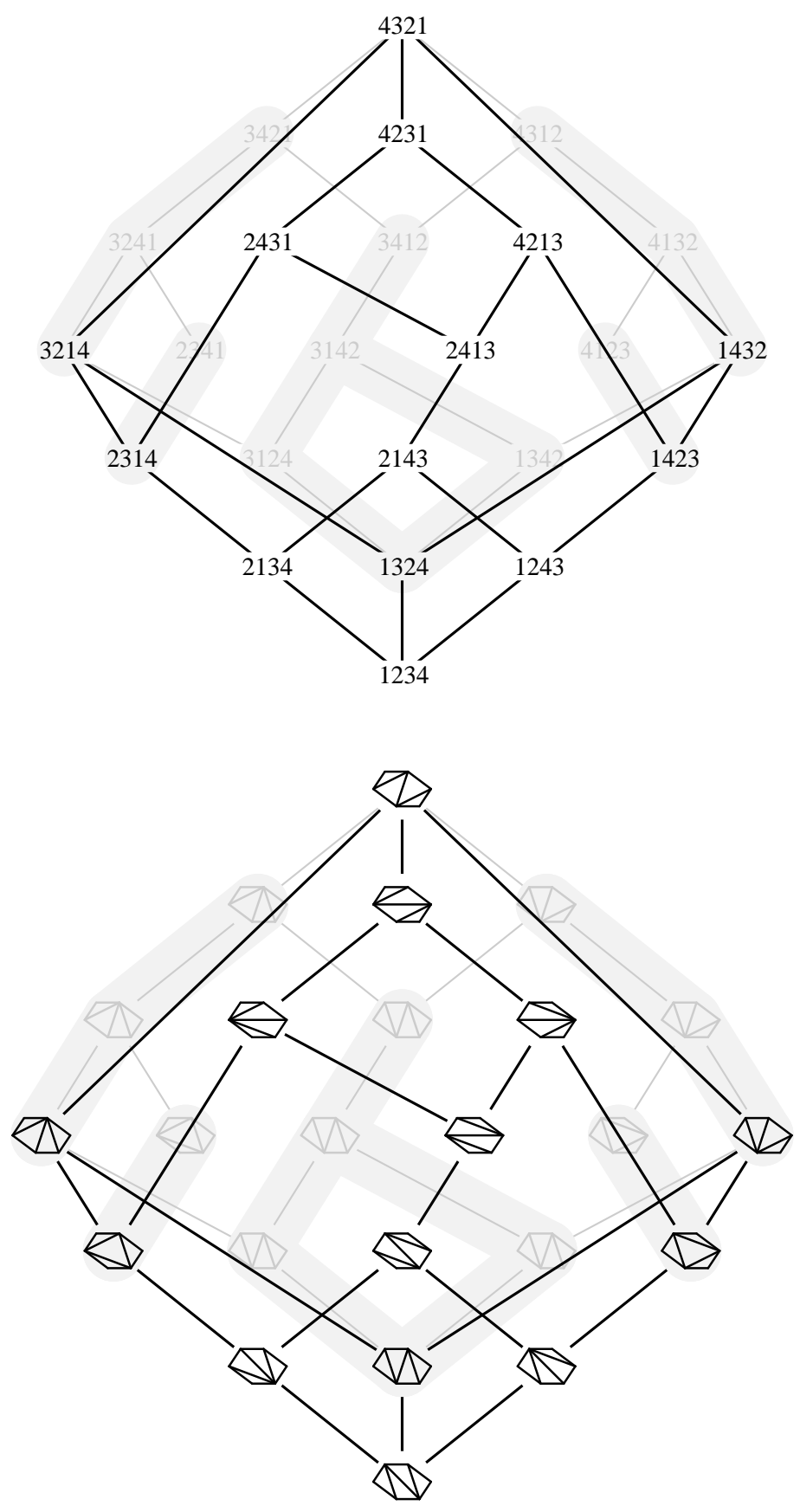

Figure 5.18. A bipartite Cambrian lattice of type $A_{3}$ 


\section{BIBLIOGRAPHY}

1. C. A. Athanasiadis, Generalized Catalan numbers, Weyl groups and arrangements of hyperplanes, Bull. London Math. Soc. 36 (2004), 294-302.

2. C. A. Athanasiadis, On a refinement of the generalized Catalan numbers for Weyl groups, Tran. Amer. Math. Soc. 357 (2005), 179-196.

3. C. A. Athanasiadis and V. Reiner, Noncrossing partitions for the group $D_{n}$, SIAM J. Discrete Math. 18 (2004), 397-417.

4. A. Berenstein, S. Fomin and A. Zelevinsky, Cluster algebras III: Upper bounds and double Bruhat cells, Duke Math. J. 126 (2005), 1-52.

5. L. Billera and B. Sturmfels, Iterated fiber polytopes, Mathematika 41 (1994), 348-363.

6. A. Björner, Orderings of Coxeter groups, Combinatorics and algebra (Boulder, Colo., 1983), 175-195, Contemp. Math. 34, Amer. Math. Soc., Providence, RI, 1984.

7. D. Bessis, The dual braid monoid, Ann. Sci. Ecole Norm. Sup. 36 (2003), 647-683.

8. R. Bott and C. Taubes, On the self-linking of knots. Topology and physics, J. Math. Phys. 35 (1994), no. 10, 5247-5287.

9. N. Bourbaki, Lie groups and Lie algebras, Chapters 4-6, Springer-Verlag, Berlin, 2002.

10. T. Brady and C. Watt, $K(\pi, 1)$ 's for Artin groups of finite type, Geom. Dedicata 94 (2002), 225-250.

11. P. Cellini and P. Papi, $a d$-nilpotent ideals of a Borel subalgebra II, J. Algebra 258 (2002), 112-121.

12. F. Chapoton, Enumerative properties of generalized associahedra, Séminaire Lotharingien de Combinatoire, B51b (2004), 16 pp.

13. F. Chapoton, S. Fomin, and A. Zelevinsky, Polytopal realizations of generalized associahedra, Canad. Math. Bull. 45 (2002), 537-566.

14. H. S. M. Coxeter, Regular polytopes, Dover Publications, Inc., New York, 1973.

15. M. Csörnyei and M. Laczkovich, Some periodic and non-periodic recursions, Monatsh. Math. 132 (2001), 215-236.

16. S. L. Devadoss, Combinatorial equivalence of real moduli spaces, Notices Amer. Math. Soc. 51 (2004), no. 6, 620-628. 
17. S. L. Devadoss, A space of cyclohedra, Discrete Comput. Geom. 29 (2003), $61-75$.

18. D. Ž. Djoković, On conjugacy classes of elements of finite order in compact or complex semisimple Lie groups, Proc. Amer. Math. Soc. 80 (1980), 181-184.

19. S. Fomin and A. Zelevinsky, $Y$-systems and generalized associahedra, Ann. of Math. 158 (2003), 977-1018.

20. S. Fomin and A. Zelevinsky, Cluster algebras I: Foundations, J. Amer. Math. Soc. 15 (2002), 497-529.

21. S. Fomin and A. Zelevinsky, Cluster algebras II: Finite type classification, Invent. Math. 154 (2003), 63-121.

22. S. Fomin and A. Zelevinsky, Cluster algebras: Notes for the CDM-03 conference, CDM 2003: Current Developments in Mathematics, International Press, 2004, 1-34.

23. S. Fomin and A. Zelevinsky, Total positivity: tests and parametrizations, Math. Intelligencer 22 (2000), 23-33.

24. V. Fock and A. Goncharov, Moduli spaces of local systems and higher Teichmuller theory, math.AG/0311149.

25. W. Fulton and J. Harris, Representation theory. A first course, SpringerVerlag, New York, 1991.

26. J. Fürlinger and J. Hofbauer, $q$-Catalan numbers. J. Combin. Theory Ser. A 40 (1985), 248-264.

27. A. Garsia and M. Haiman, A remarkable $q, t$-Catalan sequence and $q$ Lagrange inversion, J. Algebraic Combin. 5 (1996), 191-244.

28. M. Geck and G. Malle, Reflection groups. A contribution to the Handbook of Algebra, math.RT/0311012.

29. M. Gekhtman, M. Shapiro, and A. Vainshtein, Cluster algebras and WeilPetersson forms, math.QA/0309138.

30. I. Gelfand, M. Kapranov, and A. Zelevinsky, Discriminants, Resultants and Multidimensional Determinants, Birkhäuser Boston, 1994.

31. G. Grätzer, General lattice theory, 2nd edition, Birkhäuser Verlag, Basel, 1998.

32. M. D. Haiman, Conjectures on the quotient ring by diagonal invariants, J. Algebraic Combin. 3 (1994) 17-76.

33. M. Hazewinkel, W. Hesselink, D. Siersma, and F. D. Veldkamp, The ubiquity of Coxeter-Dynkin diagrams (an introduction to the A-D-E problem). Nieuw Arch. Wisk. (3) 25 (1977), no. 3, 257-307.

34. J. Humphreys, Reflection Groups and Coxeter Groups, Cambridge Univ. Press, 1990.

35. V. Kac, Infinite dimensional Lie algebras, 3rd edition, Cambridge University Press, 1990.

36. C. W. Lee, The associahedron and triangulations of the $n$-gon, European $J$. Combin. 10 (1989), no. 6, 551-560.

37. L. Lewin, Polylogarithms and associated functions, North-Holland Publishing Co., New York-Amsterdam, 1981.

38. M. Markl, Simplex, associahedron, and cyclohedron, Contemp. Math. 227 (1999), 235-265.

39. D. I. Panyushev, ad-nilpotent ideals of a Borel subalgebra: generators and duality, J. Algebra 274 (2004), 822-846. 
40. M. Picantin, Explicit presentations for the dual braid monoids, C. R. Math. Acad. Sci. Paris 334 (2002), 843-848.

41. N. Reading, Cambrian Lattices, Adv. Math., 205 (2006), no. 2, 313-353.

42. V. Reiner, Equivariant fiber polytopes. Doc. Math. 7 (2002), 113-132.

43. V. Reiner, Non-crossing partitions for classical reflection groups, Discrete Math. 177 (1997), 195-222.

44. V. Reiner and V. Welker, On the Charney-Davis and Neggers-Stanley Conjectures, J. Combin. Theory Ser. A 109 (2005), 247-280.

45. I. Reiten, Dynkin diagrams and the representation theory of algebras, Notices Amer. Math. Soc. 44 (1997), no. 5, 546-556.

46. J.-Y. Shi, The number of $\oplus$-sign types, Quart. J. Math. Oxford 48 (1997), 93-105.

47. R. Simion, A type-B associahedron, Adv. in Appl. Math. 30 (2003), 2-25.

48. E. Sommers, B-stable ideals in the nilradical of a Borel subalgebra, Canad. Math. Bull., to appear.

49. R. P. Stanley, On the number of reduced decompositions of elements of Coxeter groups, European J. Combin. 5 (1984), 359-372.

50. R. P. Stanley, Enumerative Combinatorics, vol.2, Cambridge University Press, 1999, Exercise 6.19. See also the "Catalan addendum" posted at http://www-math.mit.edu/ ${ }^{r s t a n / e c / . ~}$

51. R. P. Stanley, ibid., Exercise 6.34.

52. J. D. Stasheff, Homotopy associativity of $H$-spaces. I, II, Trans. Amer. Math. Soc. 108 (1963), 275-292, 293-312.

53. J. Stasheff, What is ... an operad? Notices Amer. Math. Soc. 51 (2004), no. $6,630-631$.

54. A. Tonks, Relating the associahedron and the permutohedron, in: Operads: Proceedings of Renaissance Conferences (Hartford, CT/Luminy, 1995), 3336, Contemp. Math. 202, Amer. Math. Soc., Providence, RI, 1997.

55. E. W. Weisstein, Archimedean Solid, in: MathWorld-A Wolfram Web Resource, http://mathworld.wolfram.com/ArchimedeanSolid.html.

56. A. Zelevinsky, From Littlewood-Richardson coefficients to cluster algebras in three lectures, Symmetric Functions 2001: Surveys of Developments and Perspectives, S. Fomin, Ed., NATO Science Series II: Mathematics, Physics and Chemistry, 74. Kluwer Academic Publishers, Dordrecht, 2002.

57. A. Zelevinsky, Cluster algebras: notes for 2004 IMCC (Chonju, Korea, August 2004), math.RT/0407414.

58. G. Ziegler, Lectures on Polytopes, Springer-Verlag, 1995.

59. J.-B. Zuber, CFT, BCFT, ADE and all that, in: Quantum symmetries in theoretical physics and mathematics (Bariloche, 2000), 233-266, Contemp. Math. 294, Amer. Math. Soc., Providence, RI, 2002. 